\title{
Development of Extreme Ultraviolet and Soft X-Ray Multilayer Optics for Scientific Studies with Femtosecond/Attosecond Sorces
}

by

Andrew Lee Aquila

B.S. (University of California, Berkeley) 2004

A dissertation submitted in partial satisfaction of the requirements for the degree of

Doctor of Philosophy

in

Engineering Science-Applied Science and Technology

in the

GRADUATE DIVISION

of the

UNIVERSITY OF CALIFORNIA, BERKELEY

Committee in charge:

Professor David Attwood, Chair

Professor Roger Falcone

Professor Stephen R. Leone

Spring 2009 
The dissertation of Andrew Lee Aquila is approved:

University of California, Berkeley

Spring 2009 
Development of Extreme Ultraviolet and Soft X-Ray Multilayer Optics for Scientific Studies with Femtosecond/Attosecond Sorces

Copyright 2009

by

Andrew Lee Aquila 


\begin{abstract}
Development of Extreme Ultraviolet and Soft X-Ray Multilayer Optics for Scientific Studies with Femtosecond/Attosecond Sorces

by

Andrew Lee Aquila

Doctor of Philosophy in Engineering Science-Applied Science and Technology

University of California, Berkeley

Professor David Attwood, Chair
\end{abstract}

The development of multilayer optics for extreme ultraviolet (EUV) radiation has led to advancements in many areas of science and technology, including materials studies, EUV lithography, water window microscopy, plasma imaging, and orbiting solar physics imaging. Recent developments in femtosecond and attosecond EUV pulse generation from sources such as high harmonic generation lasers, combined with the elemental and chemical specificity provided by EUV radiation, are opening new opportunities to study fundamental dynamic processes in materials. Critical to these efforts is the design and fabrication of multilayer optics to transport, focus, shape and image these ultra-fast pulses

This thesis describes the design, fabrication, characterization, and application of 
multilayer optics for EUV femtosecond and attosecond scientific studies. Multilayer mirrors for bandwidth control, pulse shaping and compression, tri-material multilayers, and multilayers for polarization control are described. Characterization of multilayer optics, including measurement of material optical constants, reflectivity of multilayer mirrors, and metrology of reflected phases of the multilayer, which is critical to maintaining pulse size and shape, were performed. Two applications of these multilayer mirrors are detailed in the thesis. In the first application, broad bandwidth multilayers were used to characterize and measure sub-100 attosecond pulses from a high harmonic generation source and was performed in collaboration with the Max-Planck institute for Quantum Optics and Ludwig- Maximilians University in Garching, Germany, with Professors Krausz and Kleineberg. In the second application, multilayer mirrors with polarization control are useful to study femtosecond spin dynamics in an ongoing collaboration with the T-REX group of Professor Parmigiani at Elettra in Trieste, Italy.

As new ultrafast x-ray sources become available, for example free electron lasers, the multilayer designs described in this thesis can be extended to higher photon energies, and such designs can be used with those sources to enable new scientific studies, such as molecular bonding, phonon, and spin dynamics.

Professor David Attwood Dissertation Committee Chair 
To my Mother and Father,

For their love and support. 


\section{Contents}

1 Introduction $\quad 1$

1.1 Introduction to Extreme Ultraviolet Science . . . . . . . . . . . . . . 1

1.2 Introduction to Multilayer Optics . . . . . . . . . . . . . . . . 3

1.2.1 Theory of Multilayers . . . . . . . . . . . . . . . 3

1.2.2 Reflectivity and Bandwidth .............. . . 5

1.2.3 Material Selection \& Measuring Atomic Scattering Factors . . 8

1.2.4 Multilayer Fabrication and Reflectivity Measurements . . . . . 11

1.3 Introduction to Femtosecond/Attosecond Dynamics . . . . . . . . . . 14

1.3.1 Time Scales of Physical Processes . . . . . . . . . . . . . . 14

1.3.2 Pump/Probe Techniques . . . . . . . . . . . . . . . 16

1.3.3 High Harmonic Generation . . . . . . . . . . . . . . . . . 18

1.3.4 Pulse Train and Isolated Attosecond Pulses . . . . . . . . . . 20

1.4 Thesis Overview . . . . . . . . . . . . . . . . . . . . . 22

\section{Extreme Ultraviolet Multilayers for Femtosecond/Attosecond Ap-} plications $\quad 25$

2.1 Bandwidth Limitations and Requirements . . . . . . . . . . . . . 25

2.1.1 Derivation of Bandwidth Limit . . . . . . . . . . . 26

2.1.2 Consequences of the Bandwidth Limit . . . . . . . . . . . 27

2.2 Pulse Shaping Techniques . . . . . . . . . . . . . . . . . . . . 29

2.2.1 General Pulse Shaping . . . . . . . . . . . . . . . . . 30

2.2.2 Chirp and Group Delay Dispersion . . . . . . . . . . . . . . 31

2.2.3 Optics for Pulse Shaping . . . . . . . . . . . . . . . . 35

2.3 Algorithms for Modeling \& Optimizing Aperiodic Multilayers . . . . . 38

2.3.1 Realistic Modeling of Mo/Si Multilayers . . . . . . . . . . . . 40

2.3.2 Optimization Process Using a Genetic Algorithm . . . . . . . 41

2.3.3 Sample Preparation and Reflectivity Measurements . . . . . . 46

2.4 Tri-Material Multilayers for Longer Wavelengths . . . . . . . . . . . . 49

2.4.1 Why Three Materials Can Be Better Than Two . . . . . . . . 49 
2.4.2 Material Selection, Optimization, and Fabrication of Tri-Material Coatings . . . . . . . . . . . . . . 52

2.4.3 Reflectivity Measurements . . . . . . . . . . . . . 55

2.5 Phase \& Quarter-Wave Plate Multilayers for Femtosecond/Attosecond Applications . . . . . . . . . . . . . . . . 57

2.5.1 Brewster Angle Transmission Multilayers . . . . . . . . . . . 57

2.5.2 Polarization Measurements . . . . . . . . . . . . . . . . 59

3 Pulse Compression \& Attosecond Research $\quad 64$

3.1 Phase Measurements of EUV Multilayer Optics . . . . . . . . . . . . 64

3.1.1 The Necessity of Phase Measurements . . . . . . . . . . . . 65

3.1.2 Total Electron Yield for Phase Measurement . . . . . . . . . . 67

3.2 Attosecond Pulse Measurements . . . . . . . . . . . . . . . . 73

3.2.1 Introduction to Ultrafast Pulse Measurements in the Visible and X-ray . . . . . . . . . . . . . . . . . 74

3.2.2 Pulse Measurements in the EUV . . . . . . . . . . . 78

3.2.3 Attosecond Pulse Measurements . . . . . . . . . . . . . 80

4 Applications to Femtosecond Spin Dynamics $\quad 84$

4.1 Introduction to Femtosecond Spin Dynamics . . . . . . . . . . . . . . 84

4.1.1 Models of Femtosecond Magnetics . . . . . . . . . . . . . . . 86

4.2 Comparison of Magnetic Probing Techniques . . . . . . . . . . . . . . 89

4.2.1 Magnetic Effects in the EUV/SXR . . . . . . . . . . 89

4.2.2 Free Electron Lasers Vs. High Harmonic Generators . . . . . . 92

4.3 Femtosecond Time Resolved Magnetic Measurements in the EUV . . 96

4.3.1 Experimental Setup . . . . . . . . . . . . . . . . 96

4.3.2 Optic Development . . . . . . . . . . . . . . . . 98

4.3 .3 Preliminary Results . . . . . . . . . . . . . . . . . . . 101

5 Conclusion $\quad 105$

$\begin{array}{ll}\text { Bibliography } & 108\end{array}$

$\begin{array}{lr}\text { A Analytic Modeling of Multilayers } & 129\end{array}$

B Polarization of Multilayer Quarter-Wave Plate 131

C Semi-Analytical Solution for Chirped Multilayers 134 


\section{Acknowledgments}

Foremost, I would like to express my deepest gratitude toward my thesis advisor Professor David Attwood. He has been a wonderful mentor throughout my years as an undergraduate and as a graduate student. He is the reason I chose UC Berkeley for graduate school, one of the best decisions of my life. Without Professor Attwood I do not believe I could have made it this far in my education.

I have also been fortunate to work with the multilayer group at the Center for X-ray Optics. I have never met a more dedicated and wonderful group of people. It has been a privilege to work with Eric Gullikson. Eric helped to guide my research, was a wonderful source of knowledge, and offered me my first job at the Lawrence Berkeley National Lab. Farhad Salmassi is one of the best at micro-fabrication in the world and has assisted in the fabrication of my multilayers. I have used Yanwei Liu as a sounding board for crazy ideas. I also appreciate the support, assistance and friendship from Franklin Dollar and Julia Meyer-Ilse, while working with them at the beamline 6.3.2 in the Advanced Light Source.

Throughout these past years, I have received help and support from many people at the Center for X-ray Optics and Advanced Light Source: Peter Fischer, Paul Denham, Donna Hamamoto, Seno Rekawa, Brian Hoef, Kevin Bradley, Drew Kemp, Ron Oort, Rene Delano, Patrick Naulleau, Ken Goldberg, Jeff Gamsby, Ron Tackaberry, Kristine Rosfjord, and Weilun Chao.

I would also like to thank my colleagues and collaborators; Regina Soufli, Michael 
Hofstetter, Alberto Simoncig, Naureen Ghafoor, Barbara Ressel, Professor Fulvio Parmigiani, Professor Ulf Kleineberg, Professor Ferenc Krausz, Sasa Bajt, and Eberhard Spiller. Not only has it been wonderful to work with them, they have also shown me much kindness that I appreciate deeply.

Without my fellow student and post-doc friends, graduate school would not have been as enjoyable an experience. I have enjoyed many discussions with Ryan Miyakawa, Christopher Anderson, Iacopo Mochi, Brittany McClinton, and Julian Threatt. Simi George helped in editing this dissertation. My partner in crime for many years: Anne Sakdinawat. Michael Greaves always knows what to say to make me laugh. I appreciate my officemate, Brooke Mesler Lai, she has put up with my many antics for too many years.

Lastly, I would like to thank my committee for their guidance. I have received great academic and career advice from Professor Roger Falcone. Stephen Leone's comments and suggestions have been much appreciated and have improved this work. 


\section{Chapter 1}

\section{Introduction}

\subsection{Introduction to Extreme Ultraviolet Science}

One of the last regions of the electromagnetic spectrum to be scientifically explored and technologically developed is the Extreme Ultraviolet (EUV)[1]. The photon energies spanned by the EUV are from just above the helium K 1s absorption edge (24.6 $\mathrm{eV})$ to the carbon $\mathrm{K}$ 1s absorption edge $(284.2 \mathrm{eV})[2]$. The reason this part of the electromagnetic spectrum has not been fully utilized is that all elements, and hence all materials, have absorption edges in the EUV. This places considerable constraints on optical systems for use in the EUV. All materials, including air, absorb EUV radiation. All experiments and technological applications must be in vacuum. Also creating optics to transport, focus, and image EUV radiation is dramatically more difficult compared to visible light optics. 


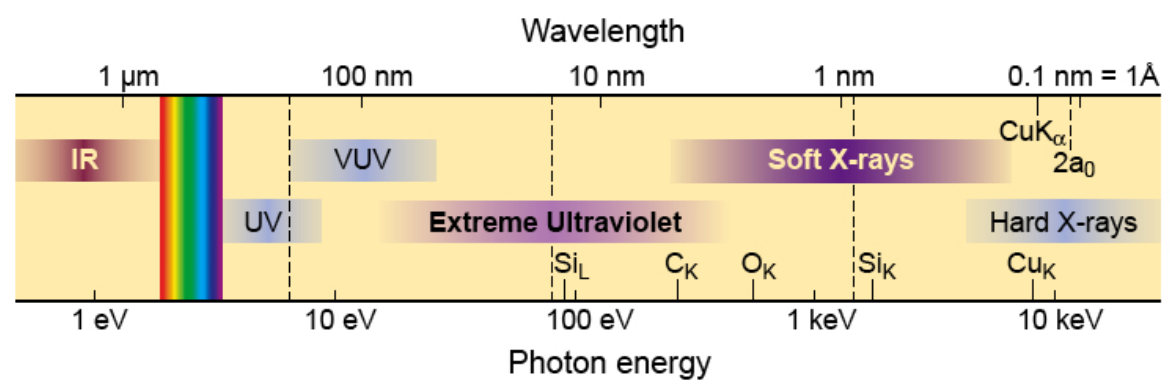

Figure 1.1: The Extreme Ultraviolet (EUV) and Soft X-ray (SXR) portions of the electromagnetic spectrum[1].

However, the disadvantages of producing optical elements in the EUV are out weighed by the advantages to scientific research. Experiments in the EUV often take advantage of the fact that all elements and many chemicals have specific absorption edges in this energy range. This allows EUV radiation to become an elemental and chemical specific probe in chemistry and material science. This is not to mention that as the wavelength of the radiation is shorter than visible light, EUV radiation is able to obtain better spatial resolution. This means that microscopes in the EUV can resolve smaller features than their visible light counterparts. These features are element specific as well, which is not the case in the visible wavelength range. Equally important, and pertaining to this work, pulses in the EUV can obtain a shorter duration than in the visible light region of the electromagnetic spectrum. Visible light pulses can obtain a few femtoseconds $\left(1 \times 10^{-15} \mathrm{~s}\right)$ in duration[3], while currently the shortest EUV pulse[3] is 80 attoseconds $\left(1 \times 10^{-18} \mathrm{~s}\right)$. This allows for the probing of atomic, magnetic and solid state processes that are impossible to observe 
with visible light.

\section{$1.2 \quad$ Introduction to Multilayer Optics}

\subsubsection{Theory of Multilayers}

Working at normal incidence in the EUV from an optics point of view is difficult compared to visible light. As stated, all materials are absorptive (i.e. have a complex index of refraction) and the real part of the index of refraction is approximately equal to 1 . This means that it is difficult or impossible to fabricate refractive lenses in the EUV. The remaining options for normal incidence are to either use diffractive optics or reflective optics. Diffractive optics such as zone plates[4][5] are used successfully as focusing/imaging elements in the EUV. There are two drawbacks in using diffractive optics. Diffractive optics are chromatic and hence suffer from chromatic aberrations. The second drawback is that they have poor efficiency. The other option, single surface reflective optics, suffer from low reflectivity at normal incidence since the real part of the index of refraction is close to one. However, there is a way to improve the normal incidence reflectivity: by creating an interference coating on top of the surface of the optic, called a multilayer[6][7][8]. Multilayers have been utilized as optical elements in EUV lithography[9] and for scientific studies such as solar physics telescopes[10].

A multilayer mirror is an interference coating composed of alternating layers of 


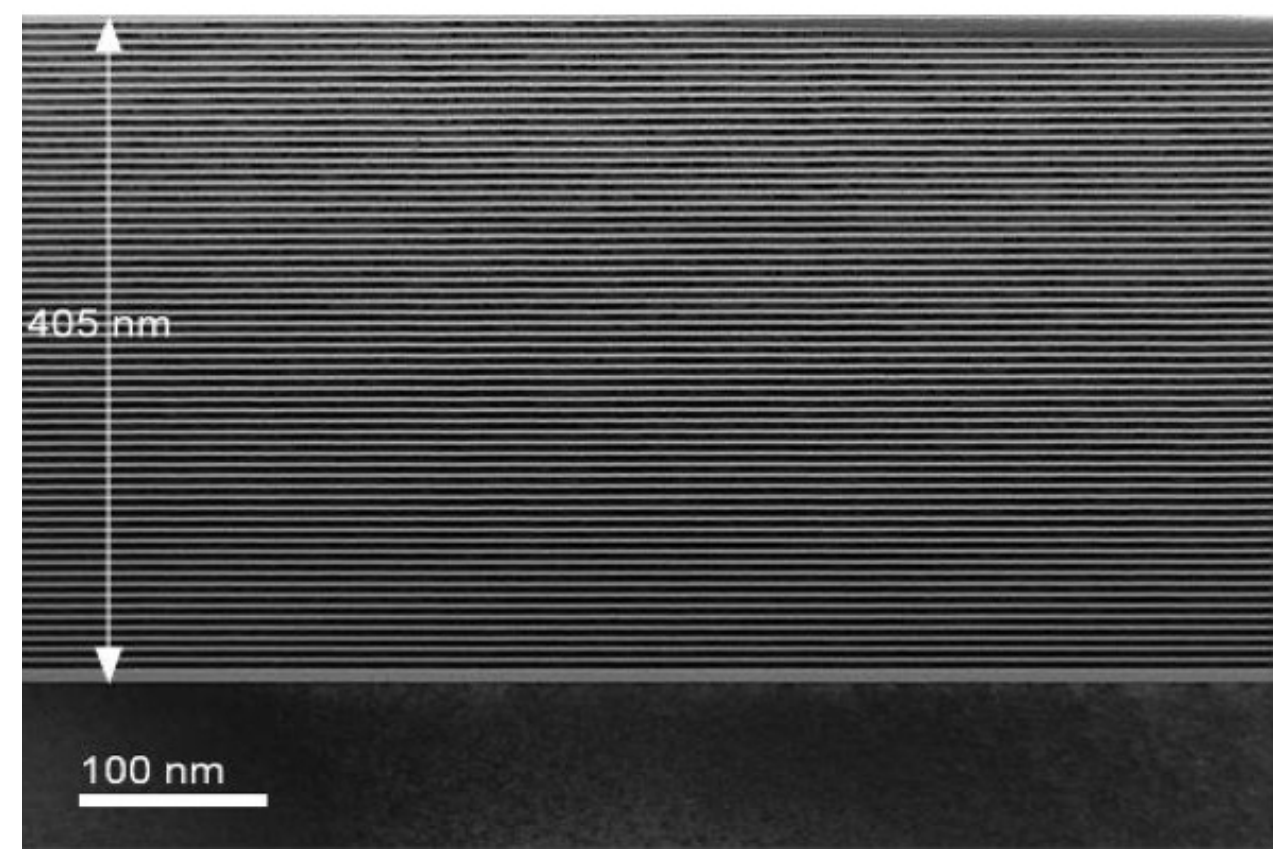

Figure 1.2: A cross-section TEM of a Molybdenum/Silicon (Mo/Si) multilayer. (Courtesy of E. Gullikson, Center of X-ray Optics)

two materials. Each layer is on the order of a few nanometers thick as seen in the transmission electron microscope (TEM) image in figure 1.2. The critical parameters of a multilayer are the number of repeating periods ${ }^{1}, N$, the thickness of one period $d$ or d-spacing, and the ratio between the bottom material of high atomic number, $\mathrm{Z}$, and the d-spacing refered to as $\gamma$. A multilayer works on the principle of temporal coherence by having the reflection from each interface add coherently with the reflections from the interface above and the interface below. This can only be achieved fully at a specific wavelength and a specific angle defined by the Bragg equation ${ }^{2}$ :

$$
m \lambda=2 d \sin \theta
$$

\footnotetext{
${ }^{1}$ Often referred to as a bi-layer.

${ }^{2}$ This formula can be corrected for the small refraction in a multilayer mirror[11]
} 


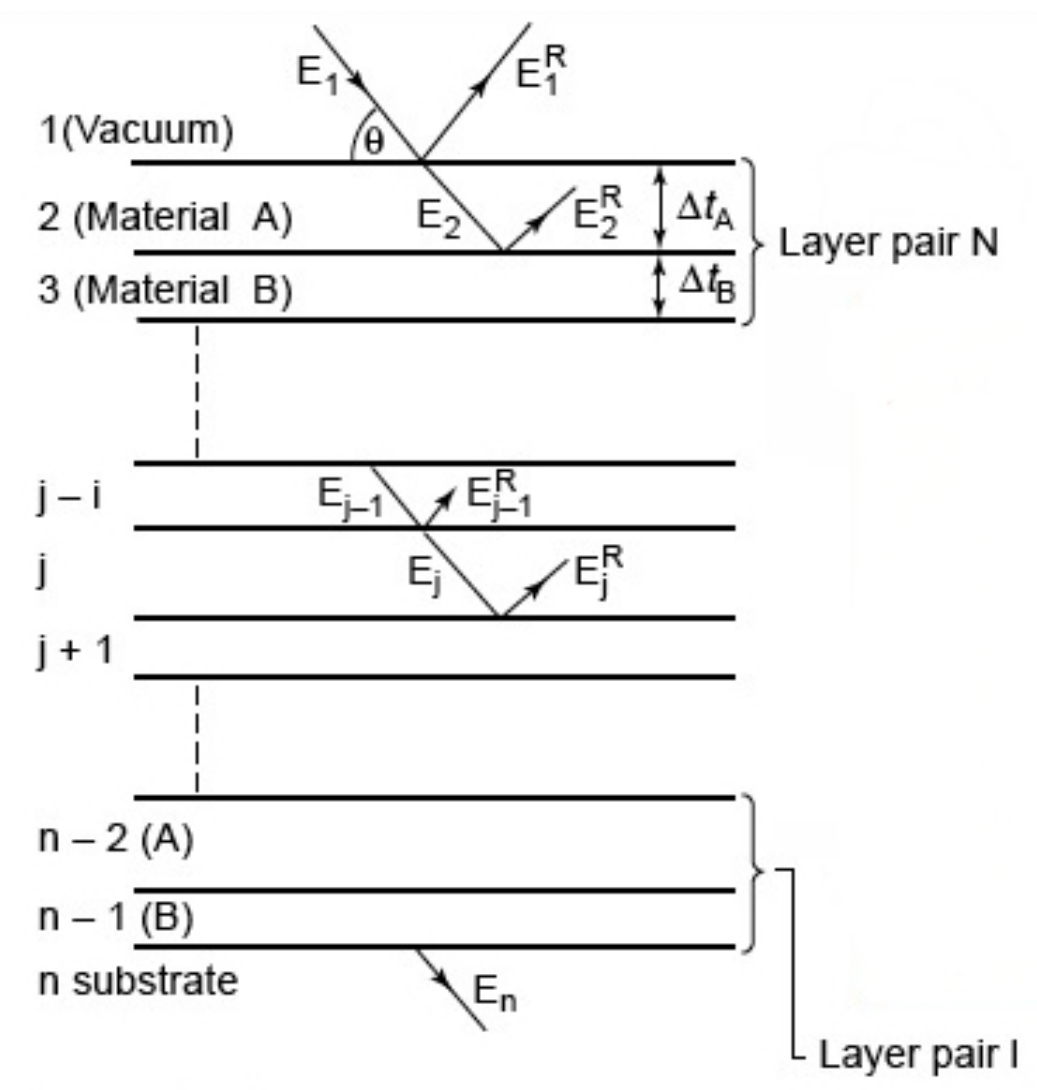

Figure 1.3: A schematic representation of a multilayer optic used to calculate the reflectivity[7].

where $m$ is the order (usually 1 ), $d$ is the d-spacing, and $\theta$ is the angle from grazing incidence.

\subsubsection{Reflectivity and Bandwidth}

If the interfaces of a multilayer optic are well defined, then the reflectivity of the multilayer optic can be calculated as a boundary value problem[7] as shown in figure 1.3. At each boundary or interface $j$, the tangental electric and magnetic fields above the interface must equal the fields below the interface. In other words, Fresnel's 


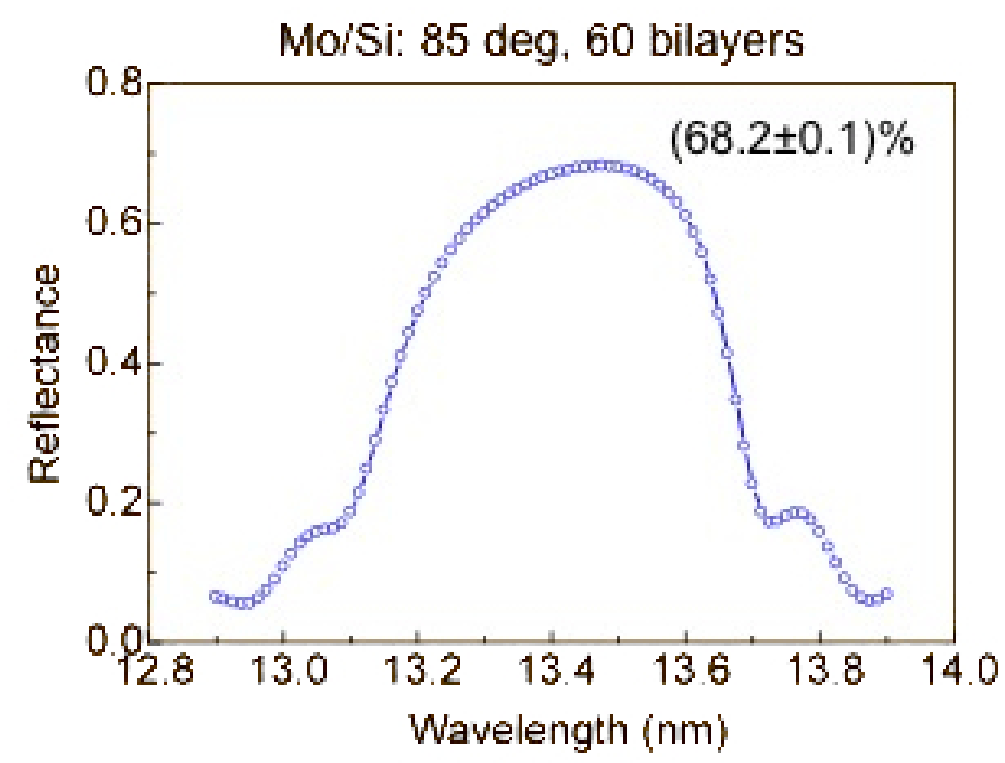

Figure 1.4: Typical reflectivity of a Mo/Si multilayer optic used in EUV Lithography. Note the parameters that describe a periodic multilayer mirror are: peak reflectivity, full width half max bandwidth, and central wavelength. (Courtesy of E. Gullikson, Center of X-ray Optics)

equations are utilized at each boundary to calculate the reflection above the boundary and the transmission below. Then, the fields are propagated through material $j$ to the boundary $j+1$. In this way we obtain $2 N+4$ variables and $2 N+2$ equations ${ }^{3}$. However, if no radiation is propagating up from the substrate in figure 1.3 and we normalize all values to the incident radiation, then we have $2 N+2$ variables and $2 N+2$ equations making the problem solvable. An example of a multilayer reflectivity curve is given in figure 1.4 .

The multilayer in figure 1.4 has a finite bandwidth and a peak reflectivity less ${ }^{3}$ The additional two equations are from the vacuum at the top interface and a substrate below
the final material. 
than 1. Careful inspection of the figure will show that the central wavelength is not the same as the peak wavelength as is often the case in the EUV. The reasons for these three characteristics is due to the optical properties of the materials that make up the multilayer. All materials absorb EUV radiation and hence have an attenuation length, thus limiting the number of usable layers. This in-turn limits the peak reflectivity and creates a finite bandwidth. Also the index of refraction is a function of the wavelength creating an asymmetric peak in the reflectivity profile. Material selection and characterization are vital for high reflectivity multilayers and for the accurate simulation of the reflectivity properties. These considerations will be discussed in section 1.2.3.

One other significant parameter for femtosecond/attosecond pulses is the required bandwidth as will be seen in section 2.1.2. An estimate for the bandwidth of a multilayer can be obtained by utilizing the fact that multilayers provides a temporally coherent responce. This means that the reflection from the lowest layer in the stack must add coherently to that of the top layer in the stack. This physical limit means that the total thickness of the multilayer is proportional to the coherence length of the multilayer:

$$
d \cdot N \approx L_{c o h}=\frac{\lambda^{2}}{2 \Delta \lambda} \Rightarrow \Delta \lambda \propto N^{-1}
$$

where $\Delta \lambda$ is the multilayer bandwidth ${ }^{4}$. Figure 1.5 shows the FWHM bandwidth and peak reflectivity of simulated Mo/Si multilayers as a function of the number of

\footnotetext{
${ }^{4}$ This proportionality is not fully correct as it neglects the effect of absorption. A more correct model would include an offset to account for the finite bandwidth of an optically thick multilayer.
} 


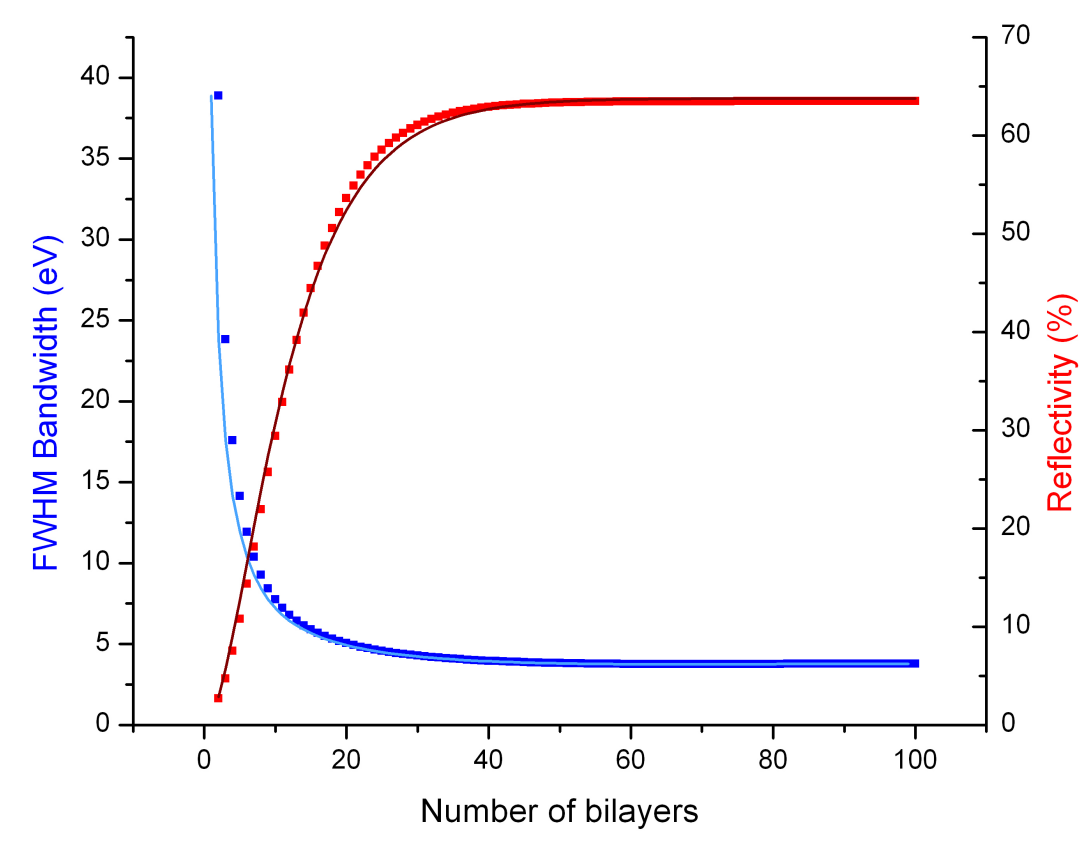

Figure 1.5: This graph shows the simulated reflectivity (red points) and corresponding full width at half maximum bandwidth (blue points) with a central photon energy of $88.25 \mathrm{eV}$. Also shown are the analytical model fits developed to describe bandwidth and reflectivity. The multilayer simulations were of a Mo/Si quarter-wave stack $(\gamma=$ 0.5 , each material has a thickness of $\lambda / 4$ ) with a d-spacing of $d=7.0 \mathrm{~nm}$; the simulation also included realistic interface modeling described in section 2.3.1. The $\gamma$ was selected to fit the analytical model described in appendix A and is not optimized for peak reflectivity.

periods.

\subsubsection{Material Selection \& Measuring Atomic Scattering Fac-}

\section{tors}

There are many factors involved in choosing materials for EUV multilayers. The largest considerations are: 1 . The complex index of refraction of the materials. 2 . 
The ability of the chosen materials to form sharp and stable interfaces. 3. The ability of the chosen materials to be fabricated. 4. The overall stability of the multilayer to time, temperature, working environment and oxidation.

While each of these factors are critical to the performance of the multilayer, often the materials selected to be reviewed and tested are chosen because of their index of refraction. The index of refraction in the EUV can be written:

$$
n=1-\delta+i \beta=1-\frac{n_{a} r_{e} \lambda^{2}}{2 \pi}\left(f_{1}^{0}-i f_{2}^{0}\right)
$$

where $1-\delta$ is the real part of the index of refraction, $\beta$ is the imaginary/absorptive part. These parameters are directly related to the complex atomic scattering factors $f^{0}(\omega)=f_{1}^{0}(\omega)-i f_{2}^{0}(\omega)$ where $n_{a}$ is the atomic density and $r_{e}=e^{2} / 4 \pi \epsilon_{0} m_{e} c^{2}$ is the classical electron radius. The attenuation of the intensity in a thin film can be calculated[1] using:

$$
I=I_{0} e^{-\frac{4 \pi \beta d}{\lambda}}
$$

as well as the normal incidence reflectivity of a material[1]:

$$
R_{\perp}=\frac{\delta^{2}+\beta^{2}}{4}
$$

The selection of materials for a multilayer consists of a low $\mathrm{Z}$ (where $\mathrm{Z}$ is the atomic number) material and a high $\mathrm{Z}$ material. The low $\mathrm{Z}$ material is often called the spacer and is chosen to have an absorption edge just above the desired wavelength. This gives the material a small $\beta$ and may have $\delta$ negative. The high $\mathrm{Z}$ material is chosen to have as large as possible difference in $\delta$ from the low $\mathrm{Z}$ material, and a 
small $\beta$ if possible. The ratio of the thickness of the high $\mathrm{Z}$ and low $\mathrm{Z}$ materials, $\gamma$ $\left(\gamma=t_{\mathrm{h}} / d\right)$, is chosen to minimize absorption, locating the thin high $\mathrm{Z}$ layers at the nodes in the standing wave inside the multilayer $\operatorname{stack}^{5}$. The optimal $\gamma$ is determined by[13]:

$$
\tan (\pi \gamma)=\pi\left[\gamma+\frac{\beta_{L}}{\beta_{H}-\beta_{L}}\right]
$$

where $\gamma$ is the optimal $\gamma, \beta_{L}$ is the $\beta$ for the low $\mathrm{Z}$ material, and $\beta_{H}$ is the $\beta$ for the high $\mathrm{Z}$ material. The optimal $\gamma$ is generally less than $1 / 2$. This is to minimize absorption. The high $\mathrm{Z}$ material is always more absorptive (larger $\beta$ ). If absorption is minimized, this increases the number of usable layers and hence increases the reflectivity. Occasionally the optimal $\gamma$ is not selected because of physical limit on achievable thickness (for example a monolayer thin film is not a realistic film thickness).

To model these materials accurately, optical constants must be known and measured. Care should be taken to obtain high resolution near absorption edges, as these are where most of the low $\mathrm{Z}$ materials will be used. As an example figure 1.6 shows the measured index of the refraction in the EUV for scandium.

To obtain these measurements, thin transmission films of different thicknesses $d$ are prepared. If the normal incident reflectivity (equation (1.5)) is negligible, as is often the case for single material thin films in the EUV, then the transmission follows from the attenuation (equation (1.4)). By using multiple thin transmission films one can obtain the values of $\beta$ or the imaginary part of the atomic scattering factor.

\footnotetext{
${ }^{5}$ Similar to the Borrman effect[12].
} 


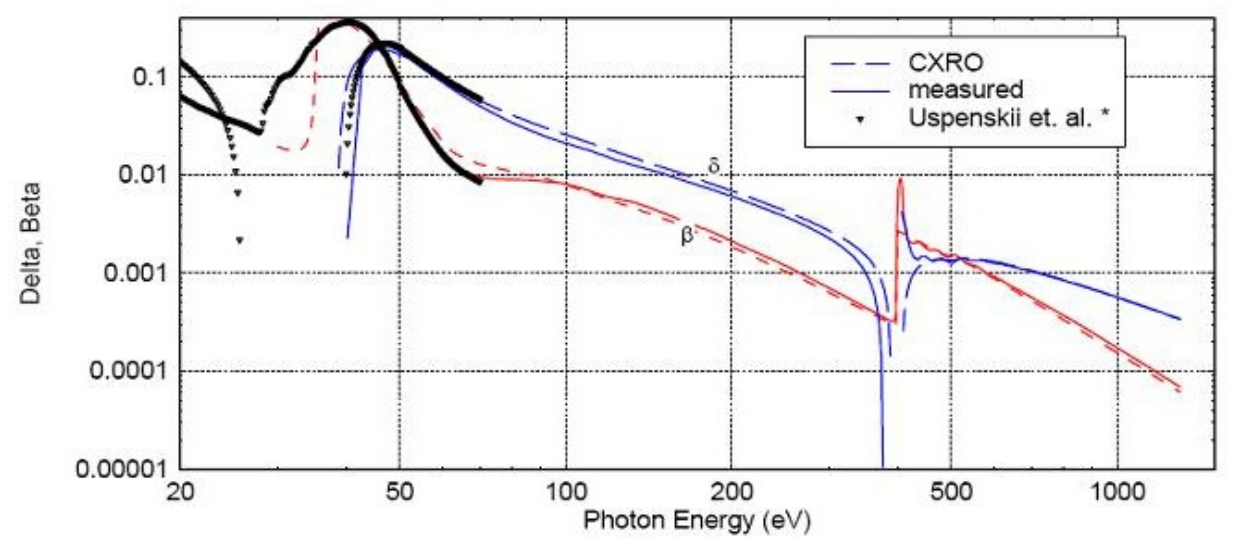

Figure 1.6: This figure shows $\delta$ and $\beta$ for scandium over the EUV and SXR range[14][15] The blue curves show $\delta$ while the red curves show $\beta$. The dashed line is the calculated values [16] while the solid lines are the measured values[14]. This material is one of the materials used in tri-material multilayers described in section 1.2.3.

Because of the linearity and causality constraints on the index of refraction, the real part, $1-\delta$, can be calculated if the imaginary part, $\beta$, of the index of refraction is known accross a broad spectrum. This is accomplished using the Kramers-Krönig relationship[17]:

$$
\delta(E)=-\frac{2}{\pi} P \int_{0}^{\infty} \frac{E^{\prime} \beta\left(E^{\prime}\right)}{E^{\prime 2}-E^{2}} \mathrm{~d} E^{\prime}
$$

where $E$ is the photon energy and $P$ represents the Cauchy principle value of the integral.

\subsubsection{Multilayer Fabrication and Reflectivity Measurements}

Multilayer coatings have been successfully fabricated using sputtering[18], electron beam evaporation[19], ion beam deposition[20], chemical vapor deposition[20], laser 
plasma deposition[21], and other methods. Direct current (DC) magnetron sputtering will be discussed as it is the method used by the author to fabricate the majority of his thin films. DC magnetron sputtering is a plasma process. A diagram of the DC magnetron sputtering system is show in figure 1.7. A plasma is produced in an evacuated chamber between the deposition material (target cathode) held at a negative voltage and a grounded rotation table (anode). Attached to the rotation table is the mirror substrate, that will be coated with the multilayer. A low pressure gas (argon) is used to produce a plasma between the target cathode and table anode. A magnetic field is placed below the target cathode to contain the plasma to the vicinity of the target cathode. The positively charged ions in the plasma are attracted to the negatively charged target cathode. The ions physically remove atoms from the target cathode when they strike it. These neutral target atoms move away from the cathode and will adhere to any surface they come in contact with. The anode table is then rotated to allow the mirror substrate to pass over the target cathode. The rotation velocity of the table is used to control the thickness of the sputtered film grown on the optic substrate. A multilayer is formed when two (or more) different target materials are used with a single rotation table. The rotation table allows the optic substrate to pass over one material followed by the other material in an alternating fashion, building up the multilayer layer coating layer by layer.

The parameters for the DC magnetron sputtering system the author use to fabricate multilayers are as follows. The system has a base pressure of better than $1 \times 10^{-7}$ 


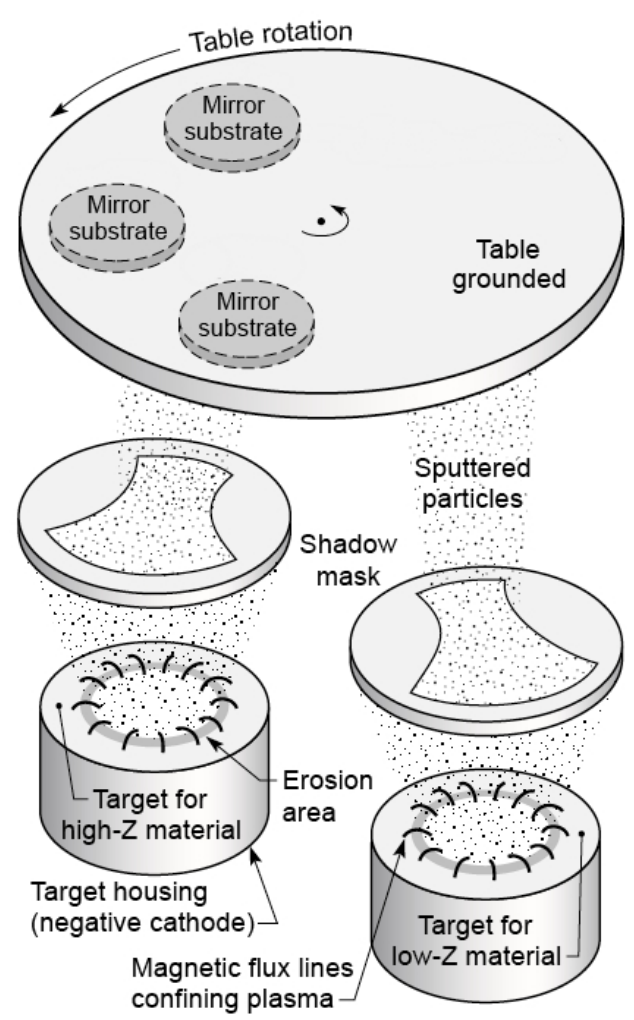

Figure 1.7: This is a diagram of the DC magnetron sputtering system described in the text. Two different sputtering targets (high $\mathrm{Z}$ and low Z) are shown below a grounded rotation table. Shadow masks are used to control the lateral distribution of materials to be deposited

Torr. The sputtering was performed with Ar gas at a pressure of 1 mTorr. The target powers were $50 \mathrm{~W}$ for Mo and $100 \mathrm{~W}$ for Si resulting in an average deposition rate of $0.59 \mathrm{~nm} / \mathrm{sec}$ for Mo and $0.83 \mathrm{~nm} / \mathrm{sec}$ for $\mathrm{Si}$. The absolute sputtering rates were measured using an alpha-step profilometer tool, grazing incidence $\mathrm{Cu} \mathrm{K}_{\alpha}$ hard x-rays, and EUV reflectometry on periodic multilayer coatings. By changing the velocity of the sample for each target we are able to control the thickness of the layer to within $1 \%$ of the desired thickness. The EUV reflectometry was measured at the Advanced 
Light Source synchrotron radiation facility.

All multilayer reflectivity measurements discussed in this dissertation, as well as the transmission filters used to experimentally determine optical constants were performed at the Advanced Light Source (ALS) calibration and standards beamline 6.3.2[22]. This beamline is a bending magnet radiation[1] beamline, designed for absolute reflectivity measurements in the EUV and soft x-ray (SXR) spectral regions. It is routinely used for multilayer EUV reflectometry measurements. The beamline has high spectral purity, and a spectral resolving power $\lambda / \Delta \lambda$ of up to 7000 , a wavelength accuracy of $10^{-3} \mathrm{~nm}$, and a reflectivity accuracy of $0.1 \%$ (absolute). The energy range of the beamline is from $25 \mathrm{eV}$ to $1,300 \mathrm{eV}$ covering the entire EUV and SXR energy ranges. These characteristics make it ideal for testing multilayer mirrors.

\subsection{Introduction to Femtosecond/Attosecond Dy-}

\section{namics}

\subsubsection{Time Scales of Physical Processes}

The fundamental time scale for the motion of molecules is the femtosecond $[23]^{6}[24]$. This timescale is the motion of the chemical bond[23] and of the vibrational states of atoms in a crystal lattice[25]. However, the EUV/x-ray spectrum offers many advan-

\footnotetext{
${ }^{6}$ The 1999 Nobel Prize for chemistry was given to Ahmed Zewail for his work in the field of femtosecond chemistry and understanding the dynamics of atomic motion. We can expect such prizes as EUV probe systems become available with the advantages of short pulse durations and element/bonding specific probes.
} 
tages over visible light femtosecond probing. The duration of pulses in the EUV can be shorter than in the visible giving a better time resolution and can lead to the capability of addressing electronic states rather than vibrational and rotational states. The spatial resolution in the EUV is better than in the visible. This becomes critical as one goes to shorter time scales because the objects under study concomitantly are smaller. Another advantage of probing in the EUV is that the higher photon energies can give structural and bond information not obtainable in the visible spectrum due to absorption edges. For example, unlike visible light EUV radiation can be used in time resolved photo-emission experiments to probe the dynamics of band structure in solids.

Much has been written about the potential of attosecond science[24][26][27][28][29][30]. All the publications on attosecond processes look at the dynamics of electrons in isolated atoms, in molecules, and in solids. The reason for this is that a bound electron has a time scale in the attosecond range. A time scale often given is the time it takes an electron to orbit a hydrogen atom[31]: $\tau_{\text {atomic }}=\hbar / 2 R y \approx 24$ attosecond (often called the atomic time scale) where one $R y$ is the binding energy of the hydrogen atom $(13.6 \mathrm{eV})$. A summary of the observable physics in a given photon energy range is shown in figure 1.8 . 


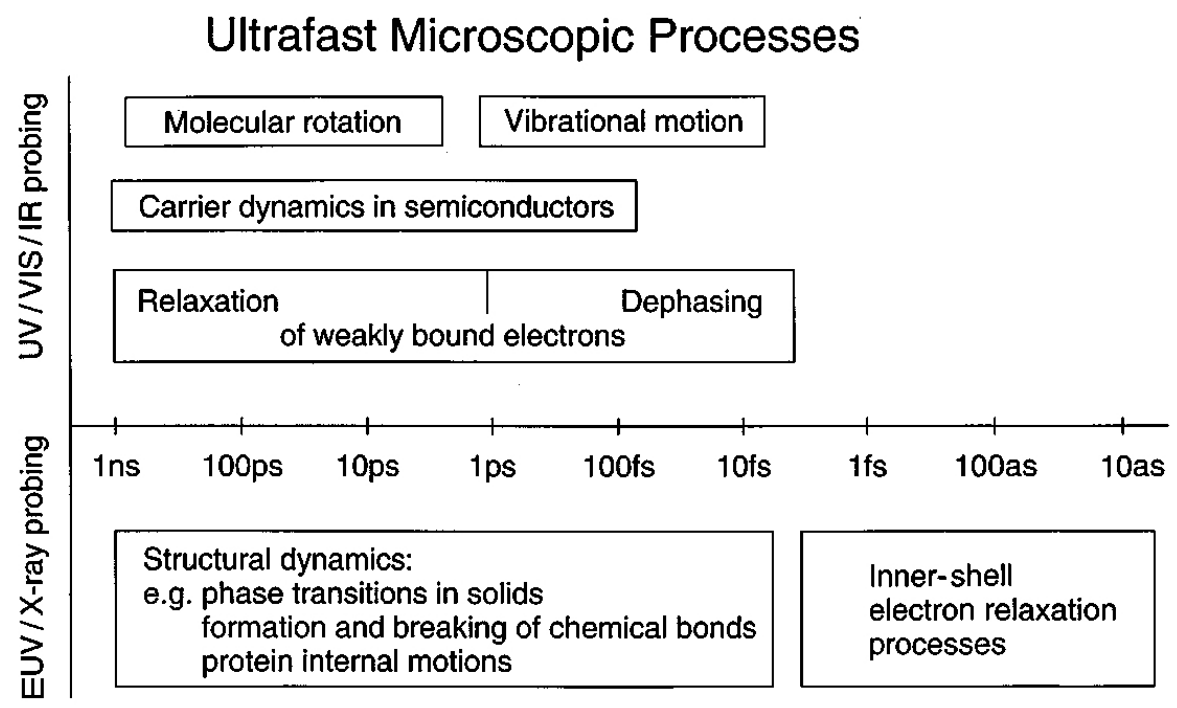

Figure 1.8: This figure shows the timescale of fundamental processes that occur in matter. The use of EUV and x-rays probe the location of the atoms and the electron dynamics on a timescale related to their motion.[24]

\subsubsection{Pump/Probe Techniques}

The basic techniques of pump and probe have not changed since the beginning of the field back in 1878 by Eadweard Muybridge[32] $]^{7}$ and by Harold Edgerton's[33] ${ }^{8}$. However, there have been orders of magnitude improvements in the observable time scales. The standard setup for a pump/probe measurement is shown in figure 1.9 of a system where the detector's readout is slower then the dynamics of the system.

A pump/probe experimental setup starts with a fast pump. This pump can be an electrical signal, an optical, infrared, or EUV pulse, or any signal that excites the

\footnotetext{
${ }^{7}$ Who made the first stop motion photographs.

${ }^{8}$ Who invented stroboscopic and flash method
} 


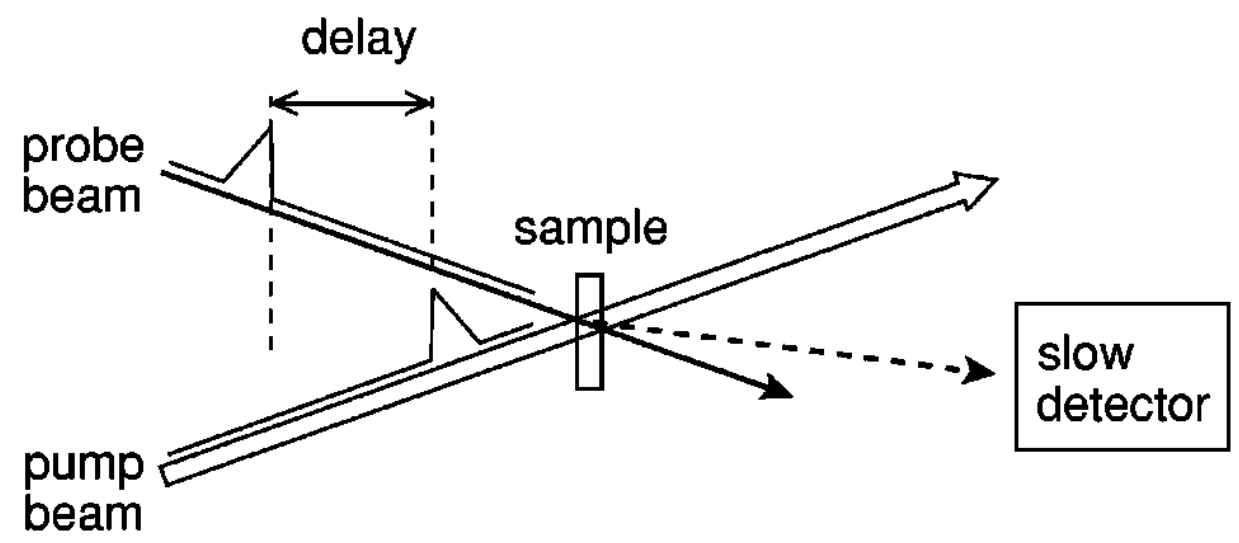

Figure 1.9: This figure shows the standard setup for a pump/probe measurement. A fast pump is used to trigger the dynamics; this pump is followed by a probe pulse. The information from the probe is then recorded on a detector. The system is then reset and repeated with the delay time between the pump and probe adjusted.[25]

desired dynamics in the sample. After a given delay, a probe pulse is used to record the dynamics of the sample on a slow detector. The probe pulse can be used in reflection, transmission, scatter, or photo-emission. This probe signal is then recorded on a detector which is then read out. The system is reset and the experiment is run again, however the time delay between the pump and probe pulses is lengthened to allow for the dynamics to progress further before being probed. In this way a record of the dynamics as a function of delay can be observed. The time resolution in a pump/probe experiment is set by the pump and probe pulses. 

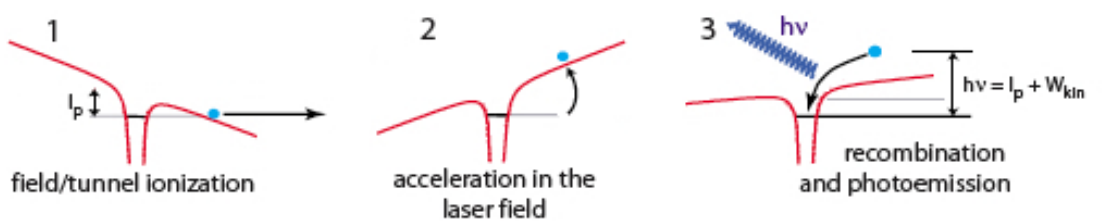

Figure 1.10: This figure gives a pictorial view of the three step model: electron tunneling, accelerating in electric field, and radiative recombination.[36].

\subsubsection{High Harmonic Generation}

The process of high harmonic generation (HHG) is the only method thus far that is used to produce attosecond EUV pulses. This subsection will give a brief review of certain aspects of the HHG process that pertain to the time structure, photon energy and polarization of HHG EUV pulses.

The HHG process can be understood from a semiclassical 3 step model[34]. (1) A strong infrared (IR) pulse is used to deform the Coulomb barrier of a gas phase atom. An electron in the atom can now tunnel through the Coulomb potential barrier of the atom. (2) The "free" electron is then accelerated classically by the field of the IR pulse. When the field reverses the electron slows down and starts to move back towards the atom. (3) The electron may then collide and recombine with the atom, giving off a photon of energy equal to the ionization potential of the atom plus the kinetic energy obtained from the IR field. Pictorially the three step process is depicted in figure 1.10. Because of the generation process the high harmonic signal also obtains the spatial coherent properties of the driving laser[35]. 
One aspect to note is that the specific moment the electron tunnels through the Coulomb field dictates the exact time it will recollide with the parent ion. This process also specifies how much kinetic energy the electron will have upon its return and when it will return. To put it another way different photon energies are produced at different times in the process. This aspect is important for producing and shaping attosecond pulses.

Another aspect to note about the process is that the driving field must be linearly polarized; otherwise, the electrons will not recombine with the ion. Using elliptically or circularly polarized light usually causes the electron trajectory to miss the parent ion $^{9}$. This also means that the EUV photons produced are also linearly polarized.

One final aspect of note to EUV science is that the photons come out in two different frequency regions: the discrete harmonic region and the cutoff continuum. This is seen in figure 1.11. The harmonic region contains the odd harmonics of the fundamental driving pulse while the cutoff is a continuum of energies and is lower in intensity than the harmonics. In the time domain, this means that the harmonic region will produce many attosecond pulses separated by a time equal to half the inverse of the driving frequency, while the continuum region will produce an isolated attosecond pulse. Details of this process will be discussed in the following section.

\footnotetext{
${ }^{9}$ There are certain circumstances where elliptically polarized light would cause recombination and produce high harmonic radiation[37].
} 


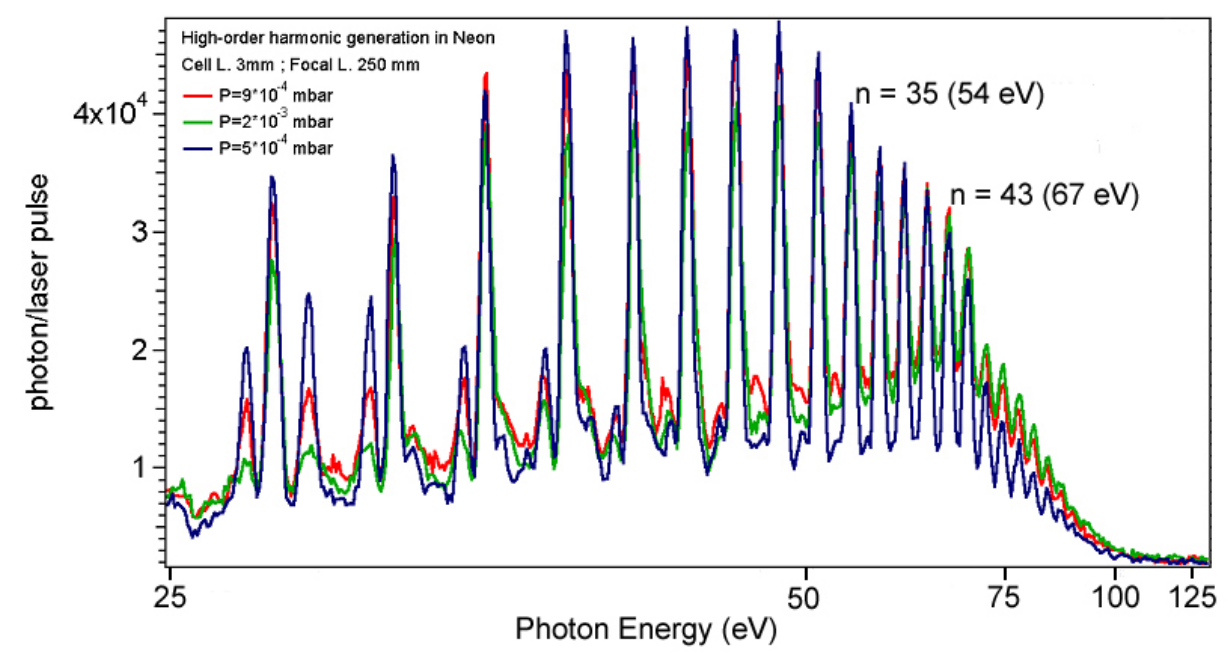

Figure 1.11: This figure is the spectrum of a high harmonic generator using neon as a generation gas. Seen clearly are the plateau of discrete harmonics and the cutoff. The driving laser was a $2 \mathrm{~mJ}$ per pulse, $800 \mathrm{~nm}$ wavelength, Ti-sapphire laser working at $1 \mathrm{kHz}$ repetition rate with, pulse duration of approximately 50 fsec. Courtesy of the T-REX lab located at the Elettra Synchrotron in Trieste, Italy. This source is used in the magnetic experiment described in Chapter 4.

\subsubsection{Pulse Train and Isolated Attosecond Pulses}

In the preceding section a qualitative description of the two parts of a HHG spectrum were described: the discrete harmonic region and the cutoff continuum. Using the second step of the three step model[34], the classical trajectories of an electron in a strong, 5 femtosecond, $800 \mathrm{~nm}$ infrared (IR) field are calculated, giving the kinetic energies of the returning electrons in the IR driving field. The produced HHG field is shown in figure 1.12. The HHG signal is produced only when the electron recombines with its parent ion. This produces a train of attosecond bursts separated by half the period of the fundamental IR field.

Shown in figure 1.12 are contributions from the two paths the electron can travel 
to recombine with the atom to produce any given photon energy[38][30]. These two trajectories have different dipole phases, therefore their phase-matching condition can be selectively enhanced by controlling the pump field.[39]. Usually the short trajectory is selected due to its small phase variation and better coherent properties. Figure 1.13 shows only the short trajectories. These trajectories can be understood as interference between the different photon energies in the HHG pulse train (both constructive and destructive). As the HHG time signal is periodic, frequency harmonics are the outcome. The harmonics will all be odd harmonics of the IR driving field as they occur twice per cycle.

Notice, also in figure 1.13, how the photon energies of the different harmonics occur at different times. This means that the harmonics produced from a HHG source are chirped ${ }^{10}$. The peak harmonic energy also changes as a function of the driving field. This has a slight modulating effect on the bandwidth of the harmonic signal. More importantly, above a certain photon energy there is no more harmonic interference. This means that there is neither constructive or destructive interference in this regime. As such, the photons above a certain photon energy are in a continuum. If this bandwidth (termed isolated pulse bandwidth in figure 1.13) is filtered, an isolated attoseond pulse is produced. In this simulation, a cosine carrier envelope phase $(\mathrm{CEP})^{11}$ is used. A stable cosine $\mathrm{CEP}$ with few cycles is one of the methods that produces an isolated attosecond pulse[3].

\footnotetext{
${ }^{10} \mathrm{~A}$ chirped pulse is longer than a pulse with zero chirp. A complete description will be given in section 2.2.2.

${ }^{11} \mathrm{~A}$ complete description of CEP will be given in section 2.2.2.
} 


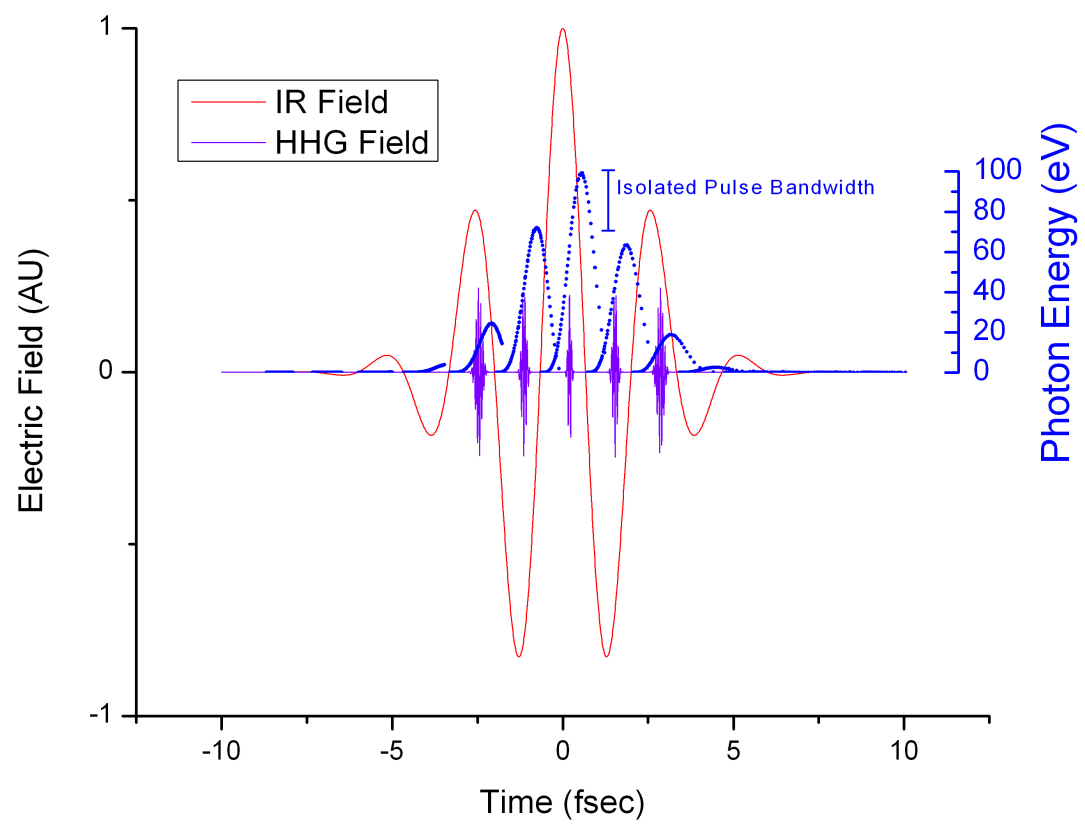

Figure 1.12: This figure shows the energies of the HHG process (blue dots with photon energy scale on the right) as a function of when they are produced in time. The photon energies were calculated using classical trajectories of an electron in the electric field of an IR pulse (red curve), producing a train of HHG pulses (violet curve). The simulation assumes neon with an IR intensity of $4 \times 10^{14} \mathrm{~W} / \mathrm{cm}^{2}$, at 800 nm wavelength and 5 femtosecond pulse duration.

\subsection{Thesis Overview}

This thesis details the development of optics and metrologies for utilizing femtosecond/attosecond EUV pulses. The second chapter of this thesis focuses on the development of optics to shape and utilize femtosecond/attosecond EUV pulses in experiments. Specifically, a detailed analysis of why EUV radiation is necessary to obtain sub-femtosecond pulses, and the shaping of femtosecond/attosecond pulses are 


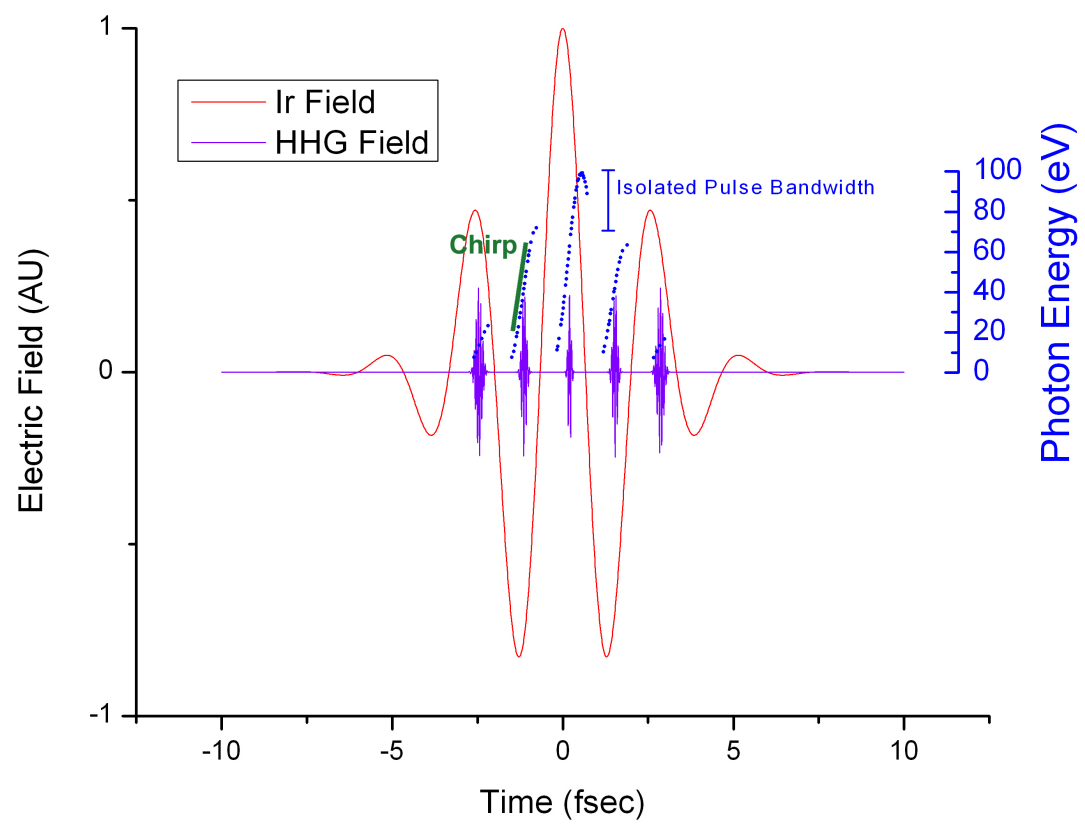

Figure 1.13: This is a modification of figure 1.12 showing only the short trajectories. Note that different photon energy (blue dots) occur at different emission times (labeled Chirp in the figure). This causes the attosecond pulses to be chirped. Also notice the center attosecond pulse contains photon energies not found in the neighboring attosecond pulses (labeled Isolated Pulse Bandwidth). If this bandwidth is filtered, an isolated attosecond pulse is produced.

described. This is followed by the development of aperiodic multilayers for bandwidth and pulse control. Chapter 2 concludes with examples of tri-layered multilayers for improved bandwidth and reflectivity, and transmission multilayers for polarization control.

Chapter 3 develops methods for measuring attosecond pulses. This includes methods used for measuring the reflected phase of a multilayer optic. This method becomes useful in determining how the EUV optical system will affect the shape and duration 
of the EUV pulse. The rest of chapter 3 describes measurement techniques for EUV pulses and the measurement of attosecond pulses.

Chapter 4 describes ongoing efforts to perform a time resolved EUV magnetooptical experiment. The experiment is an attempt to probe the limits of how quickly a magnet responds to a femtosecond IR heating pulse. The experiment is an attempt to understand the mechanism and timescale of spin dynamics interactions in a solid. 


\section{Chapter 2}

\section{Extreme Ultraviolet Multilayers}

\section{for Femtosecond/Attosecond}

\section{Applications}

\subsection{Bandwidth Limitations and Requirements}

To date most scientific work in the EUV/SXR/x-ray region has been essentially "static"; limited by the $\sim 100$ psec duration of radiation at modern synchrotron facilities. Now at the dawn of new femtosecond/attosecond sources, High Harmonic Generators (HHGs) and Free Electron Lasers (FELs), we are entering a new and exciting domain of dynamical studies, on time scales of the order of atomic bonding, vibrational and spin dynamics. When considering ultrashort pulses, the temporal 
structure and spectral bandwidth are of great concern to the experiment being performed. For example, in a pump probe dynamics experiment the time resolution of the system is limited by the pump and probe pulse durations. Reducing the duration of these pulses concomitantly improves the time resolution of the system and hence allows for the ability to see faster dynamics. However, nature puts a physical bandwidth limitation on how short an electromagnetic pulse can be.

\subsubsection{Derivation of Bandwidth Limit}

All pulses of finite duration have a spread in frequency; this holds true for all electromagnetic radiation. The electric field of a light pulse can be described by an amplitude function multiplied by an oscillating central frequency. An example is that of a Gaussian light pulse:

$$
E(t)=e^{\frac{-t^{2}}{2 \sigma^{2}}} e^{-i \omega_{0} t}
$$

where $t$ is time, $\omega_{0}$ is the central frequency and $\sigma$ is the root mean square measure of the pulse duration. The full width half maximum (FWHM) duration of the pulse can be calculated from the intensity by:

$$
\begin{gathered}
I(t) \propto|E(t)|^{2}=e^{\frac{-t^{2}}{\sigma^{2}}}=\frac{1}{2} \\
\Delta \tau_{F W H M}=t_{+}-t_{-}=2 \sqrt{\ln 2} \sigma=1.67 \sigma
\end{gathered}
$$

where $t_{+}$and $t_{-}$are the positive and negative roots of the half maximum value in peak intensity. To obtain the frequency spectrum of the pulse a Fourier transform of 
the electric field is taken:

$$
\begin{gathered}
E(\omega)=\frac{1}{\sqrt{2 \pi}} \int_{-\infty}^{\infty} e^{\frac{-t^{2}}{2 \sigma^{2}}} e^{-i \omega_{0} t} e^{i \omega t} \mathrm{~d} t \\
E(\omega)=\sigma e^{-\frac{\sigma^{2}}{2}\left(\omega-\omega_{0}\right)^{2}}
\end{gathered}
$$

Solving for the FWHM intensity spread in frequency, we obtain:

$$
\Delta \omega_{F W H M}=\frac{2 \sqrt{\ln 2}}{\sigma}
$$

Solving for $\sigma$ in (2.3) and inserting it into (2.6) we obtain:

$$
\Delta \omega \Delta \tau=4 \ln 2=2.77
$$

We can place this into convenient units of femtoseconds for time and electron volts (eV) for energy (by multiplying by $\hbar$ ) we obtain:

$$
[\Delta E \Delta \tau]_{F W H M}=1.825 \mathrm{eV} \cdot \mathrm{fs}
$$

This result on the required bandwidth does depend on the profile of the pulse. This result can also be obtained from the Heisenberg Uncertainty Principle in the time/energy domain[40]. This gives the limit of:

$$
[\Delta E \Delta \tau]_{F W H M} \geq 1.825 \mathrm{eV} \cdot \mathrm{fs}
$$

\subsubsection{Consequences of the Bandwidth Limit}

The immediate consequence of the time/bandwidth requirement on pulses is that to obtain attosecond pulses, the central wavelength of the pulse must be in the EUV or 


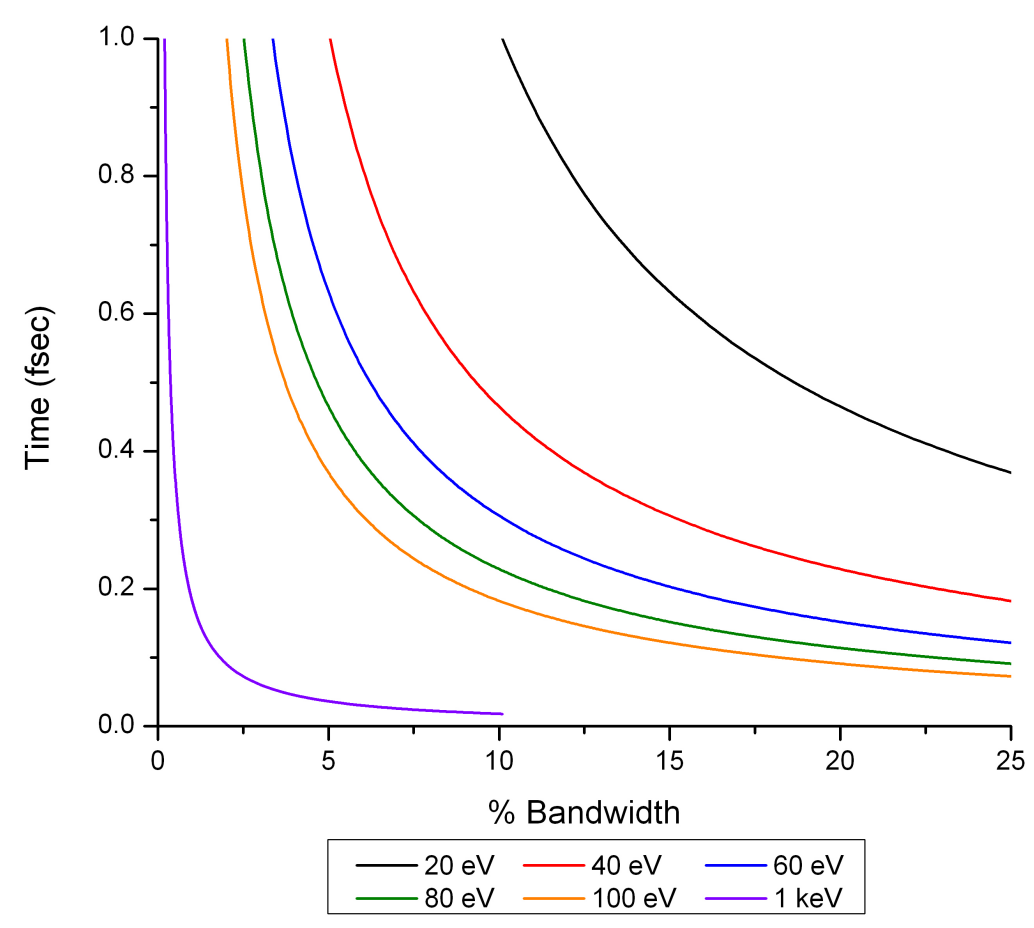

Figure 2.1: This graph shows the minimum relative bandwidth, $\Delta E_{\mathrm{FWHM}} / E_{\mathrm{cent}}$, requirement for sub-femtosecond pulses as derived from equation 2.9.

the x-ray region of the electromagnetic spectrum. Meaning it is physically impossible for visible light pulses to obtain sub-femtosecond duration. The reason for this is easy to show by plotting the relative bandwidth requirement, $\Delta E_{\mathrm{FWHM}} / E_{\text {cent }}$, of the pulse verses pulse duration, as in figure 2.1. Where $\Delta E_{\mathrm{FWHM}}$ is the full width at half max bandwidth of the pulse and $E_{\text {cent }}$ is the center (peak) photon energy of the pulse. As the pulse duration is reduced, the required bandwidth is increased. It becomes physically impossible to obtain a pulse with $\Delta E_{\mathrm{FWHM}} / E_{\text {cent }}>2$ (from 0 frequency to twice the central frequency $)^{1}$. Often the limiting factor in bandwidth is not the source

\footnotetext{
${ }^{1}$ This is an over estimate as the pulse would still require frequencies beyond the FWHM values.
} 
duration but that of the optical system involved in the transport, focusing, shaping, and imaging of the pulse. Thus developing optics that preserve pulse duration is critical.

In the EUV a 100 attosecond pulse centered at $90 \mathrm{eV}$ (the ideal range for Mo/Si multilayers) requires a bandwidth of order $20 \mathrm{eV}$. This required bandwidth would prevent the usage of diffractive optics due to dispersion ${ }^{2}$. It would also limit the peak reflectivity of a standard periodic multilayer optic due to the bandwidth restrictions discussed in section 1.2.2.

\subsection{Pulse Shaping Techniques}

Even if a pulse has a broad bandwidth does not mean that it will be short in duration. Along with bandwidth considerations, often times the shape of the pulse, or pulses, matters greatly to the experiment being preformed. These considerations can be broken into two types: amplitude and phase. Amplitude considerations describe what frequencies are included in a pulse. For example a non-Gaussian pulse shape will have a larger pulse duration than a Gaussian pulse with the same FWHM bandwidth, since the non-Gaussian pulse is not band-width limited. In contrast, phase considerations describe how the different frequency components of a pulse relate to each other. One phase consideration is chirp, a chirped pulse will always have a larger

\footnotetext{
${ }^{2}$ Unless two or more diffractive optics were used to compensate for pulse spreading and dispersion as will be seen in section 2.2.1. However, in the EUV the efficiency of such a system would be very low.
} 
bandwidth than an non-chirped pulse. Both considerations are important in shaping EUV pulses and can be developed under the general theory of pulse shaping.

\subsubsection{General Pulse Shaping}

An optical system for pulse shaping is a linear system $[41]^{3}$. This means that you can think of a pulse of light in the time domain, and the reflection off the optic as a convolution of the pulse with the mirror impulse response function. The alternative is to view both the pulse and optic in frequency space. In the frequency domain a pulse is multiplied by the frequency response of the optical system as seen in figure 2.2. Any given frequency component in a pulse can be thought of as consisting of an amplitude and a phase value:

$$
E(\omega)=|E(\omega)| e^{i \phi(\omega)}
$$

The $|E(\omega)|$ is easily understood as the frequency components that are part of the pulse. The phase components are important for determining the duration of a pulse and will be discussed in the next subsection.

\footnotetext{
${ }^{3}$ There are a few non-linear exceptions[42], but in the EUV this statement is valid, as will be shown in the next chapter.
} 

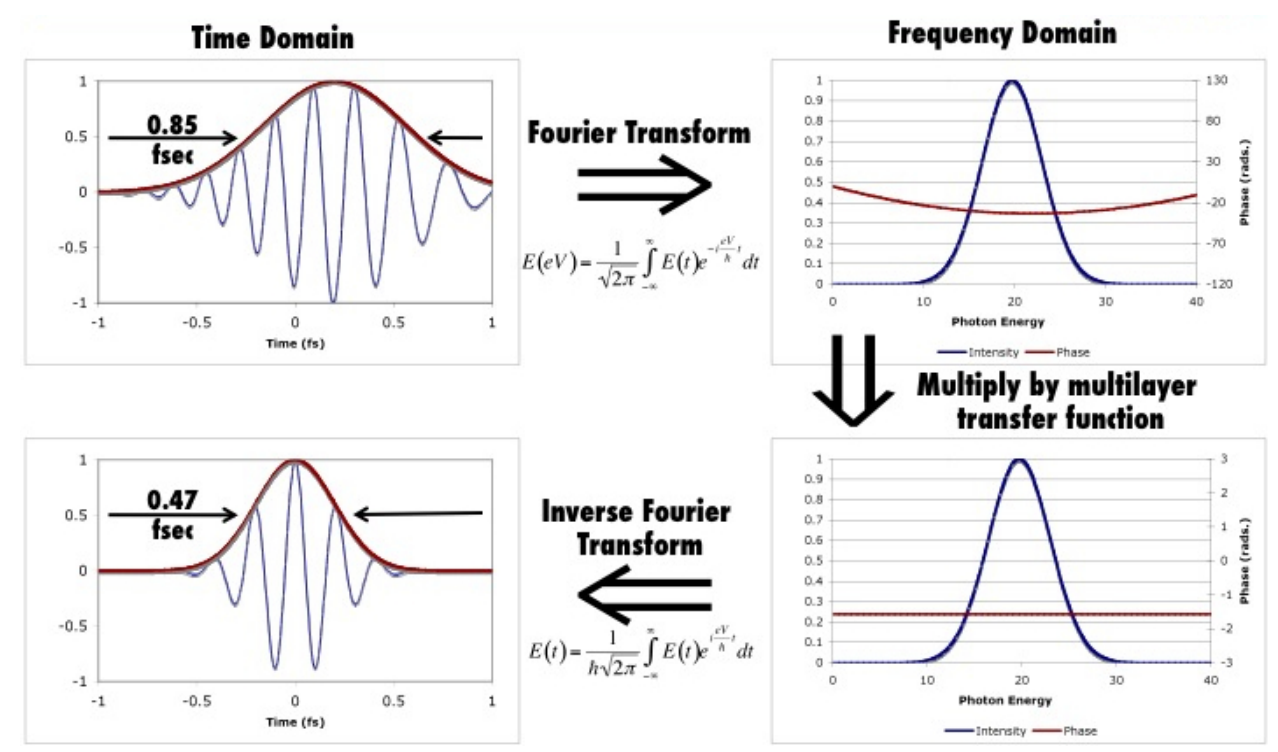

Figure 2.2: This figure shows the relation between the time domain and the frequency domain for shaping EUV pulses. The upper left figure is a chirped EUV pulse (a Gaussian envelope with a quadratic phase). The upper right figure is the frequency domain showing both amplitude (blue) and phase (red). The lower right image show the effects of the optical system (a multilayer mirror that removes the quadratic phase). The lower left shows the time domain image of the newly shaped pulse (blue is the electric field and red is the intensity envelope).

\subsubsection{Chirp and Group Delay Dispersion}

The phase component $\phi(\omega)$ in equation (2.10) can often be approximated by a Taylor series expansion around the central frequency $\omega_{\text {cent }}[43]$ :

$$
\begin{gathered}
\phi(\omega)=\sum_{n} \frac{1}{n !} \phi^{n}\left(\omega_{\text {cent }}\right)\left(\omega-\omega_{\text {cent }}\right)^{n} \\
\phi(\omega)=\phi\left(\omega_{\text {cent }}\right)+\phi^{\prime}\left(\omega_{\text {cent }}\right)\left(\omega-\omega_{\text {cent }}\right)+\phi^{\prime \prime}\left(\omega_{\text {cent }}\right) \frac{\left(\omega-\omega_{\text {cent }}\right)^{2}}{2 !}+\cdots
\end{gathered}
$$

The first term in the expansion $\phi\left(\omega_{\text {cent }}\right)$ is called the carrier envelope phase (CEP).

This term determines, for example, if the pulse is a sine wave or a cosine wave. For example, a 200 attosecond cosine pulse with a central frequency $\hbar \omega_{\text {cent }}=45 \mathrm{eV}$ is 
shown in figure 2.3, while the same pulse with a sine carrier envelope phase is shown in figure 2.4. For attosecond pulses, this term is usually not as important as it has no effect on the pulse size ${ }^{4}$ for all pulses.

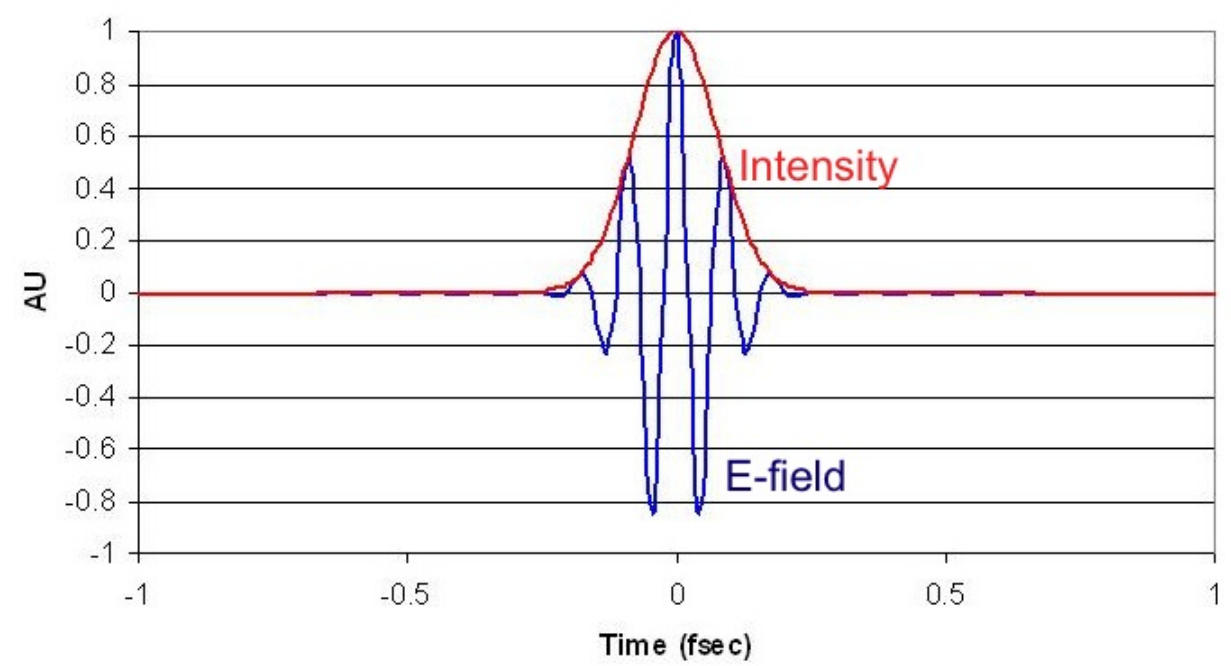

Figure 2.3: This graph shows a 200 attosecond bandwidth limited Gaussian cosine carrier envelope phase (CEP), pulse with a central energy of $45 \mathrm{eV}$. The electric field is shown in blue and the intensity envelope is shown in red.

The second term $\phi^{\prime}\left(\omega_{\text {cent }}\right)$ (first derivative) in the expansion of equation $(2.12)$ is called the group delay (GD). This term is linear in frequency. The effect of changing the GD term in the expansion shifts/delays the pulse in time, as seen in figure 2.5 . This term preserves the shape and duration of the pulse. The group delay is not of great concern in attosecond pulses, as it can be changed my moving the position of the optics.

\footnotetext{
${ }^{4}$ For nonlinear processes such as the creation of isolated attosecond pulses the CEP of the driving infrared laser does matter
} 


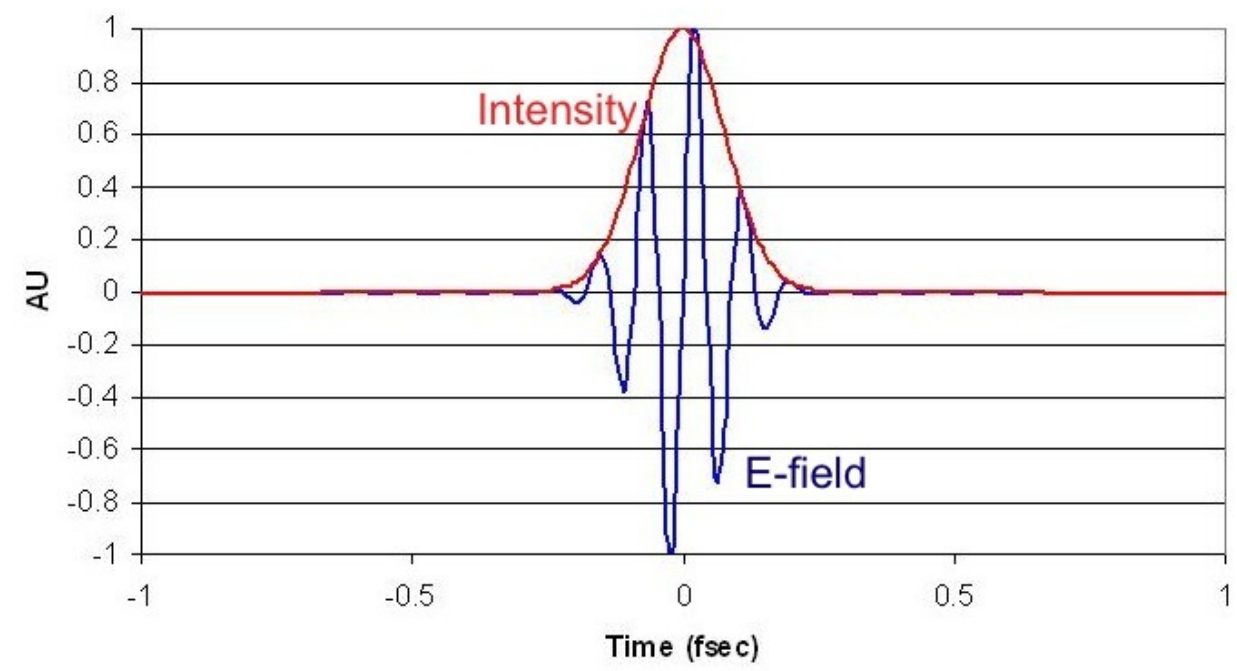

Figure 2.4: This graph shows the same pulse in figure 2.3 with a sine CEP.

The third term in the series expansion of equation (2.12) (second derivative of the phase), $\phi^{\prime \prime}\left(\omega_{\text {cent }}\right)$, is called the group delay dispersion (GDD). A pulse with a GDD is always longer than a pulse with zero GDD, as can be seen in figure 2.6 , where the pulse has the same bandwidth as figure 2.3. However, it is significantly (factor of 4) larger than 200 attoseconds.

A pulse with a GGD is also called a chirped pulse. Chirp can be thought of as the rate of change of frequency within a pulse. A positive chirp begins at a lower frequency and finishes the pulse at a higher frequency. A negatively chirped pulse finishes on a lower frequency than the frequency it began with. Extending the derivation given in section 2.1.1 it can be shown that the duration of a pulse containing GDD is[43]:

$$
\tau=\tau_{0} \sqrt{1+\frac{16(\ln 2)^{2}\left\{\phi^{\prime \prime}\left(\omega_{\text {cent }}\right)\right\}^{2}}{\tau_{0}^{4}}}
$$




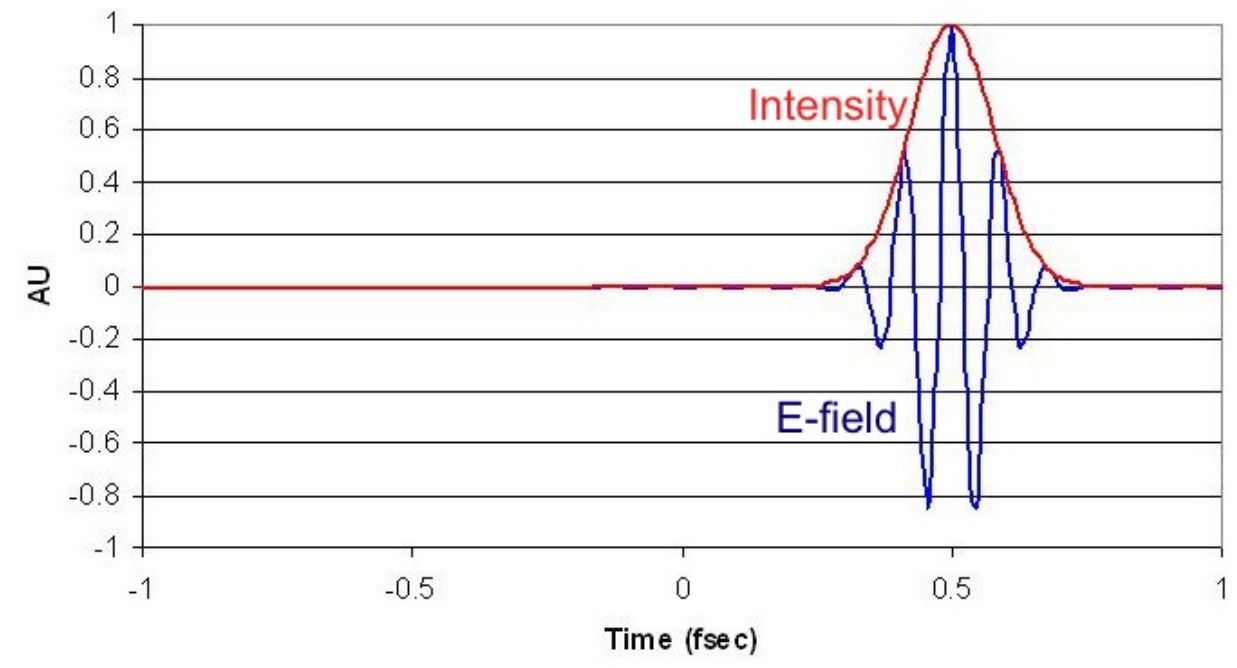

Figure 2.5: This graph shows same pulse in figure 2.3 with a group delay (GD) of 0.5 femtoseconds.

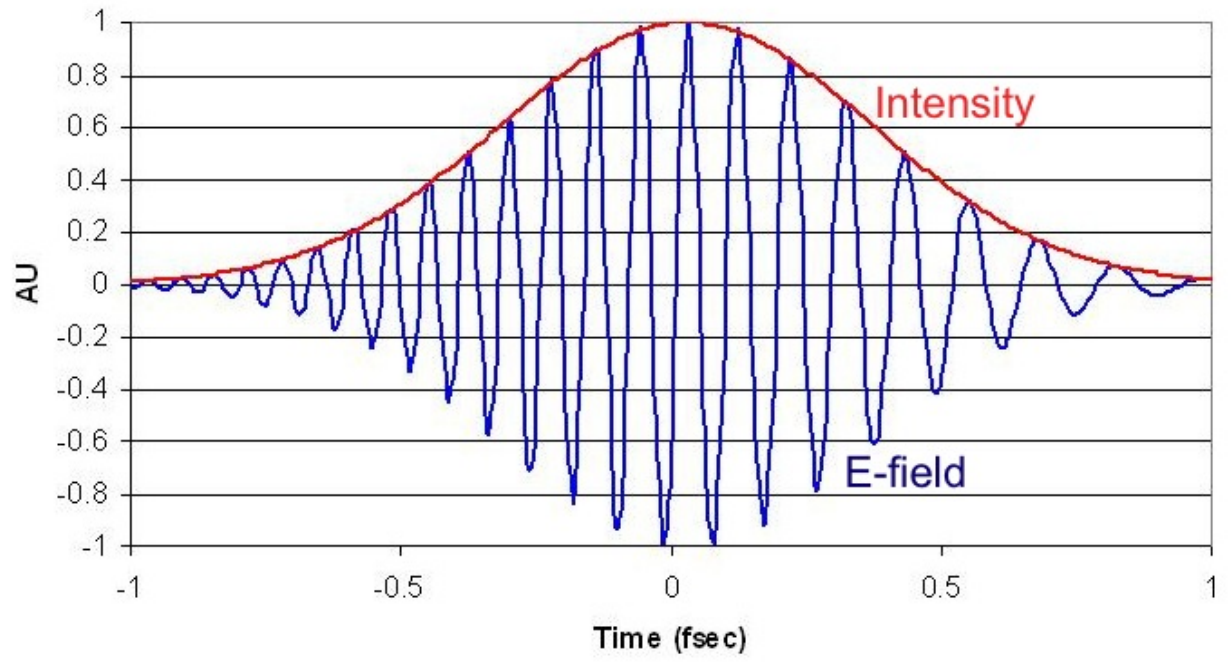

Figure 2.6: This graph shows same pulse in figure 2.3 with a group delay dispersion (GDD) of 0.047 femtoseconds $^{2}$. Notice how the electric field moves from a high frequency to a low frequency across the pulse. 
where $\tau$ is the pulse duration with GDD $\phi^{\prime \prime}\left(\omega_{\text {cent }}\right), \tau_{0}$ is the original pulse duration without GDD obtained from (2.9). One interesting effect to note is the $\tau_{0}^{4}$ in the denominator. This means that for a fixed GGD a shorter pulse will be chirped more than a longer pulse.

\subsubsection{Optics for Pulse Shaping}

All pulse shaping techniques either change the relative amplitude of the different frequency components of the pulse or the relative phases between the different spectral components in the pulse. Changing the amplitude amounts to filtering the spectral content of the pulse. In the visible light this is done by dispersion of the light off a grating or through a prism Then the light is dispersed through another prism or lens (used to eliminate the angular frequency dependent divergence from the first grating/prism) to produce a Fourier plane where each frequency component is separated in space and can be filtered individually. Then the frequency components are brought back through the reverse optical system as shown in figure 2.7. In the EUV, this type of system is not fully practical as it would require four gratings and a patterned filter mask making the efficiency low. However, amplitude shaping can be either accomplished using filters near absorption edges or designing optical elements to filter out specific frequencies as will be discussed in section 2.3.

There are two methods to adjust the relative phases of the various frequency components in a pulse. Both methods work on the idea of a simple equation, $t=d / V_{\phi}$, 


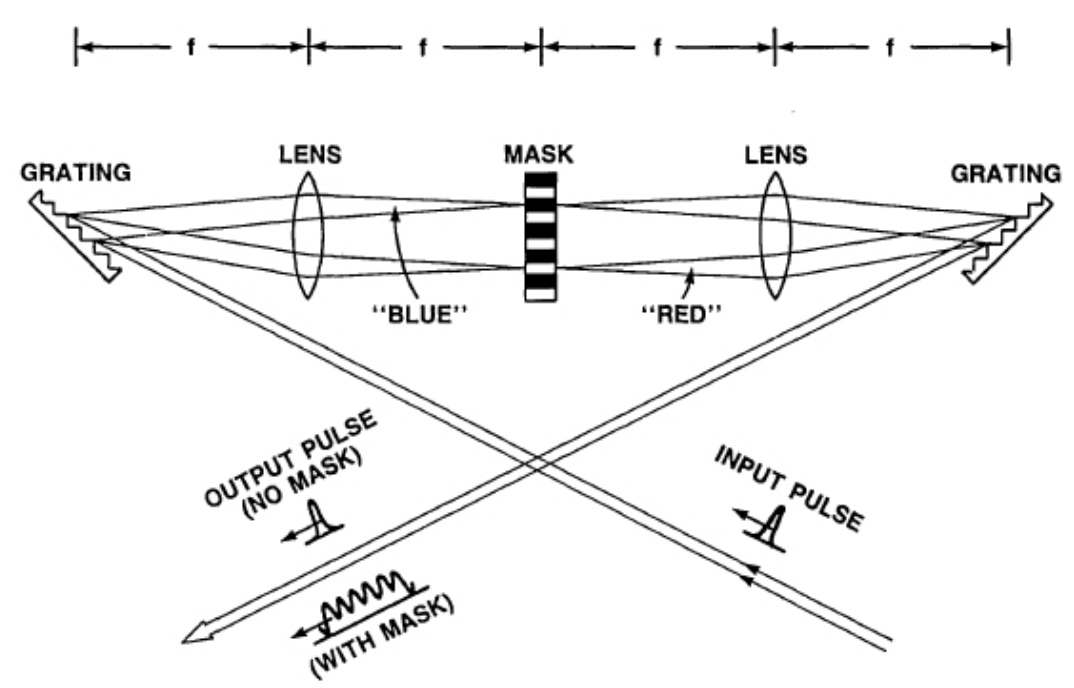

Figure 2.7: This image shows a general system for shaping femtosecond light pulses. The pulses are dispersed from a grating followed by a lens creating a plane that maps frequency to position. At this plane a patterned filter mask modulates the amplitude and phase of the individual frequency components. The pulse is the recombined using a lens and another grating [44].

where $t$ is the desired time shift, $d$ is the path length traveled and $V_{\phi}$ is the phase velocity of the specific component. In vacuum $V_{\phi}=c$. If one wants to move a specific frequency component slightly in time with respect to another, then either the path length $d$ of one frequency must be longer than the other or the phase velocity between the two frequency components must be different.

One method that uses the difference in phase velocity is material dispersion[45]. This method relies on the index of refraction of a material and is photon energy/wavelength dependent. This means that the various frequencies in a pulse propagate at different velocities in the material. The phase change as a function of thickness[46] is:

$$
\Delta \phi(\omega)=-\omega \delta(\omega) d / c
$$


This method has two limitations. The first is the thickness of the material used. Due to absorption limits on all material one is limited in thickness of the filter, and hence limited in the dispersion compensation. The second limitation is that all materials used must be used below an energy absorption edge; this assures maximum transmission through the filter. However this requirement limits the usefulness, as filters can only compensate for only pulses with negative dispersion. This is because right below an energy absorption edge $\delta$ has a negative slope.

The second method for pulse compression is to use different path lengths for different frequency components. In the visible and infrared regions of the electromagnetic spectrum this is achieved using four prisms[47] or four gratings. The first grating angularly disperses the radiation. This adds a negative GDD[48]. The second grating is placed to optimize the path length difference to compensate for the negative GDD, and it is used to compensate for the angular spread caused by the first grating. If the light pulse in lateral size is much larger than the size of the dispersed spectrum the chirp free pulse can be obtained directly after the second grating. If however this is not the case then two more gratings need to be used to eliminate the spatial dispersion. These methods can be implemented in the EUV, but due to low efficiency, difficulty in alignment, and as easier alternative methods are available they are normally not used.

An alternative method that obtains higher efficiencies and easier alignment that is used in visible light[49][50][51] and in the EUV[52][53] is to depth grade a multilayer 

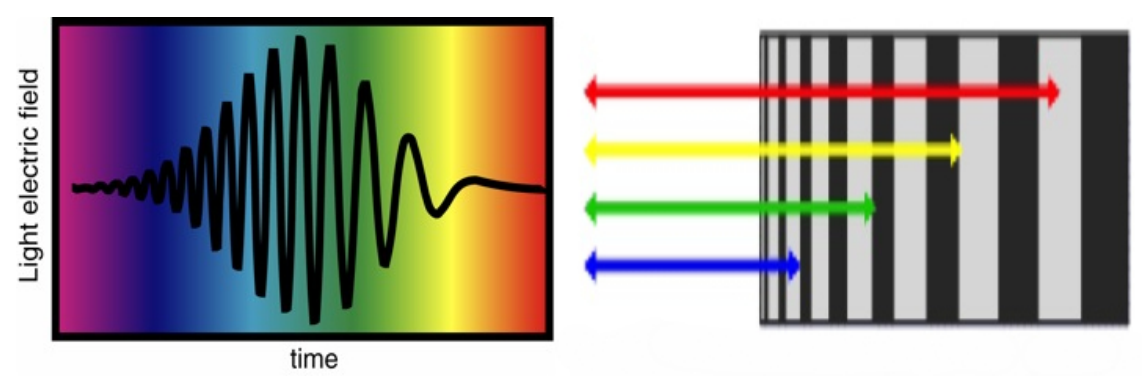

Figure 2.8: This image shows a multilayer mirror uses to correct for the GGD of an incident pulse. The different frequency components satisfy different locations or depths in the multilayer. Hence they suffer different time shifts and can correct for GDD.

mirror. This is seen in figure 2.8. The idea is that the different frequency components are reflected at different locations in the multilayer where the radiation locally satisfies the Bragg condition. Depth graded multilayers will give a path length difference and hence a phase difference between the different spectral components of the pulse. By measuring the phase difference between the incident radiation and the reflected radiation at a given wavelength, one can determine the effect of the multilayer on shaping the pulse.

\subsection{Algorithms for Modeling \& Optimizing Ape- riodic Multilayers}

One method of achieving larger bandwidths while maintaining high reflectivity is to use aperiodic multilayers. Aperiodic multilayer structures have been developed[54][55] for various applications that require larger energy bandpasses than can be achieved 
with periodic structures. These structures have been used with great success under the title of supermirrors in both neutron optics, and grazing incidence hard x-ray optics. However, the use of aperiodic multilayers in large angle SXR/EUV optics has been hampered due to lack of agreement in the optimizing simulations compared to the deposited multilayer films on the optics. Often the measured reflectivity can differ significantly from the design goal. While these differences could result from the inaccuracies in the deposition process there is also the predictable error caused by the intermixing at the multilayer interfaces. To implement EUV optics for femtosecond/attosecond pulses realistic modeling of interfaces must be taken into consideration, along with development of nonlinear optimization algorithms for multilayers. This section concludes with a demonstration of the success of this work by implementing a large square bandpass aperiodic multilayer.

The Mo/Si multilayer system is probably the best studied system due to its importance as a high reflectance normal incidence coating for EUV lithography and astronomy. Mo/Si multilayer optics are also well suited for femtosecond/attosecond pulses, as the optical constants for molybdenum and silicon provide an operational range (where Mo/Si obtains high reflectivity) from 70-100 eV. This matches the range HHG sources work at, and creates a suitable bandwidth for attosecond pulses. Additionally the interface formation of $\mathrm{MoSi}_{2}$ is well understood. When the formation of $\mathrm{MoSi}_{2}$ is properly included in the design of an aperiodic multilayer structure, it is possible to fabricate a multilayer with a performance very close to the design. For these 
reasons the author chose to use the $\mathrm{Mo} / \mathrm{Si}$ multilayers to demonstrate the techniques uses to shape femtosecond/attosecond pulses.

\subsubsection{Realistic Modeling of Mo/Si Multilayers}

It is well known that the intertermixing of the Mo and Si at the interfaces plays an important role in determining the structure of a Mo/Si multilayer. The multilayer not only becomes a three-component system but also contracts as the Mo and Si are consumed in the interface layer changing the multilayer period. This is illustrated in figure 2.9 for an aperiodic structure where the left most image is what would be expected without considering the intermixing. The center image is the expected structure when intermixing is taken into account, as described below. The right image is a TEM of the fabricated multilayer. The interdiffused silicide regions in standard Mo/Si multilayers have been observed to be asymmetric with the silicide layer being thicker for the Mo deposited on a Si interface than for the Si deposited on a Mo interface. Measurements[56] indicate that the inter-diffused layer is amorphous with a stochiometry of nearly $\mathrm{MoSi}_{2}$. An amorphous to crystalline transition occurs in the Mo layer for a thickness around $2 \mathrm{~nm}$; for comparison a quarter-wave $\operatorname{stack}^{5}$ with peak reflectivity at $92 \mathrm{eV}$ has a Mo thickness of $3.4 \mathrm{~nm}$. This transition results in a significant decrease in the silicide layer for the $\mathrm{Si}$ on Mo interface[57]. In our modeling, it is assumed that the interface layers are $\mathrm{MoSi}_{2}$. For the Mo on Si interface,

\footnotetext{
${ }^{5} \gamma=0.5$
} 
the silicide layer thickness increases from $0.6 \mathrm{~nm}$ to $0.95 \mathrm{~nm}$ as the Mo is deposited[56]. For Si on Mo, the silicide layer is assumed to be $0.95 \mathrm{~nm}$ when the deposited Mo layer is thinner than the critical thickness of $2.0 \mathrm{~nm}$ and $0.6 \mathrm{~nm}$ if the deposited Mo thickness is greater than $2.0 \mathrm{~nm}$. For each nanometer of $\mathrm{MoSi}_{2}$ that is formed, it takes $1 \mathrm{~nm}$ of Si and $0.39 \mathrm{~nm}$ of Mo, resulting in a contraction of $39 \%$ of the silicide layer thickness. This contraction reduces the overall thickness of the multilayer stack changing the d-spacing of each layer and thus its wavelength dependent reflectivity. It is noted that the interfacial composition and thickness will vary between different deposition methods; we choose interdiffusion parameters based on measurements conducted and published in the literature, for DC magnetorn sputtering to insure the realism of the simulation.

\subsubsection{Optimization Process Using a Genetic Algorithm}

In this work, multilayer mirrors were optimized using a single parent, mutation based, genetic algorithm[58] also called a Luus-Jaakola algorithm[59]. The genetic algorithm process takes a seed or parent multilayer composed of a stack of $\mathrm{Mo} / \mathrm{Si}$ pairs; the thickness of each Mo or Si layer is considered a gene. Small Gaussian random thickness variations, or mutations, were added or subtracted from each gene in the parent multilayer stack; the exception is that we set a lower bound on the thickness of any layer to $1 \mathrm{~nm}$. These new variations formed the children of the parent multilayer. In this specific optimization 100s of children were used due to the 


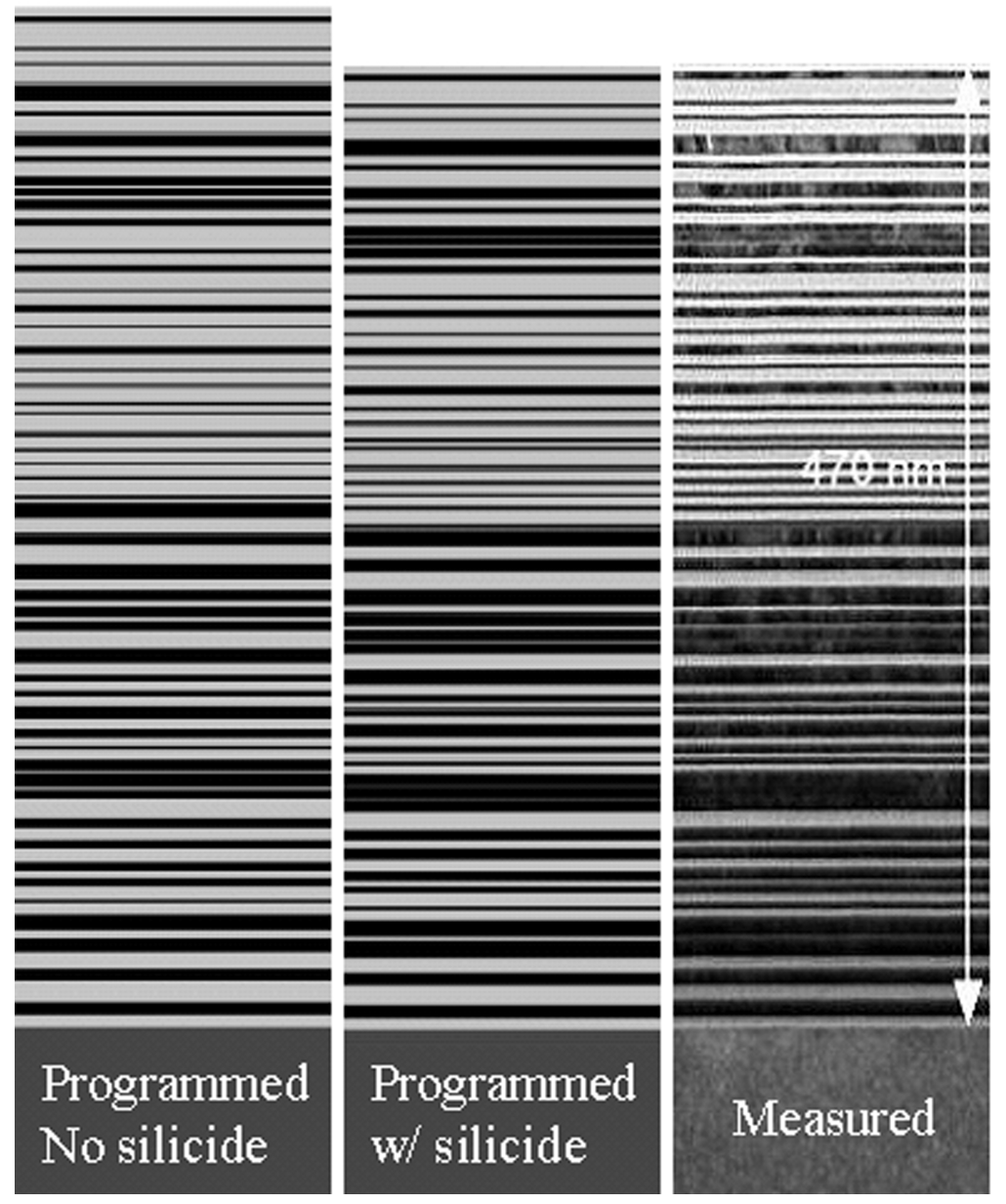

Figure 2.9: Simulation of the programmed Mo and Si thickness (Left); modeling the contraction caused by $\mathrm{MoSi}_{2}$ formation at the interfaces (Center); actual TEM crosssection of the fabricated multilayer (Right). This demonstrates that including the silicide formation is required to match the simulation to the actual sputtered sample. The programmed thickness ranged from $1.2 \mathrm{~nm}$ to $10.3 \mathrm{~nm}$ for silicon and $1.4 \mathrm{~nm}$ to $8.2 \mathrm{~nm}$ for molybdenum.

large number of genes. To put it another way we are considering points in and on a sphere centered at the parent with radius the size of the thickness variation in 120 dimensional space. We then simulated the effects of $\mathrm{MoSi}_{2}$ : the contraction of the multilayer and the roughness between each layer of the child multilayer. Each of the 
children are evaluated for reflectivity using standard methods[19][7] and by giving each child a numerical ranking. A merit function process chooses the numerical ranking where the wavelength dependent reflectivity was compared against a desired function:

$$
M=\int\left(R_{m}(\lambda)-R_{c}(\lambda)\right)^{2} \mathrm{~d} \lambda
$$

where $R_{m}$ is the merit function or desired reflectivity curve, $R_{c}$ is the simulated childs multilayer reflectivity curve, and $\mathrm{M}$ is the minimization parameter or numerical ranking. The child with the smallest M became the new parent for the next generation. This generational process is repeated until all the children of the parent multilayer have larger values of $\mathrm{M}$ than the parent. This occurs when the multilayer parameters have drifted to a minimum. Then the size of the mutations is reduced, and more generations are simulated. This process stops when the size of the mutations is reduced to a value below the coating tolerance of our deposition machine. At this point we have an optimized reflectivity coating.

It is noted that the genetic optimization method does not guarantee the absolute global optimization, the method is slower than many other optimization algorithms, and it also dependent on the initial seed multilayer or starting condition. The algorithm was chosen as it solves large parameter space nonlinear problems, it is simple to conceptualize, easy to code and its has the ability to be run in parallel unlike simulated annealing algorithms[60]. With computational time being relatively inexpensive, the speed of the algorithm is less of a critical factor compared to the results 
produced by the optimization. With a genetic algorithm a global optimization can often be reached with this algorithm if an optimal seed is used.

For our specific samples a 60 or 40 bi-layer stack of Mo/Si was used. The 60 bi-layer pair was initially chosen to ensure that we obtained an optically thick mirror, however it soon became apparent that we could remove the bottom 10 to 20 layers without significantly affecting the reflectivity. This made an organism composed of 120 , or 80 , ordered genes with each gene being a number representing the thickness of the layer in the multilayer and the gene number representing the position of the layer in the stack. The current seed multilayer is a standard periodic Mo/Si stack, this is a non-optimized seed multilayer for large bandpasses. Using a pre-developed analytic solution [61] can create optimal seed multilayers, however current analytic solutions have only been developed for two component systems. Simulating the growth of $\mathrm{MoSi}_{2}$ and the contraction of the multilayer in these solutions diminishes its usability.

The author found that an easy way to obtain a nearly ideal seed is to first create a polynomially depth grated multilayer in both d-spacing and $\gamma$. The author also used the layer number, $n$, as a free parameter. For example, if a cubic function was used 
in d-spacing, a linear function in $\gamma$, then this would be a 7 parameter fit:

$$
\begin{array}{r}
d[n]=a n^{3}+b n^{2}+c n+g \\
\gamma[n]=e n+f \\
d_{M o}[n]=\gamma[n] * d[n] \\
d_{S i}[n]=(1-\gamma[n]) * d[n]
\end{array}
$$

where $a, b, c, g, e, f, n_{\max }$ are parameters. Because of the number and nature of this reduced parameter space either the brute force $\operatorname{method}^{6}$ or the linear simplex algorithm[60] are used. These polynomial multilayers are used as optimized seeds in the genetic algorithm to insure a global optimization is reached. When looking at solutions developed using genetic algorithms it is often difficult to determine why the solution is an optimization or if the optimization is a global optimization. Using different starting conditions can lead to the possibility of obtaining different optimizations. Also, due to the fact that the children are based on random mutations if an insufficient number of children is used then different local optimizations can be achieved with the same starting conditions. If an optimal seed and sufficient children are used then the global optimization can be obtained. Looking at figure 2.9, the bottom half of the multilayer looks intuitively incorrect due to the larger thickness of Mo layers that act as absorbers. After conducting simulations with the bottom layers removed it became clear that only the top 80 to 100 layers play a role in the simulation the rest of the layers would be below the attenuation length and have no effect. Also the sharp edge

\footnotetext{
${ }^{6}$ Solve all possible values and combination of the parameters.
} 
bandpass structure develops in the top 40 layers consisting of three large reflectivity peaks with large fluctuation. The remaining layers acting to flatten out the top of the reflectivity curve.

\subsubsection{Sample Preparation and Reflectivity Measurements}

The multilayers were coated using DC magnetron sputtering and measured at the ALS beamline 6.3.2 as described in 1.2.4. In order to demonstrate the importance of the silicide formation, two 45-degree mirrors were designed, one that included the silicide interface layers and the other that did not. Both mirrors were designed with the same output goal parameters $\left(R_{m}\right)$ : a bandwidth of $3 \mathrm{~nm}(18.3 \mathrm{eV})$ centered at $14.25 \mathrm{~nm}(87 \mathrm{eV})$ with an average reflectivity of $20 \%$ within the bandpass and $0 \%$ reflectivity outside this bandpass. The bandwidth parameter was chosen to correspond to a 100 attosecond pulse, while the square shape was arbitrarily selected to demonstrate the method's ability to perform amplitude shaping on the input spectrum of a pulse.

The results for the multilayer that was designed without taking the silicide layers into account is shown in figure 2.10. This mirror consisted of 40 bi-layers (80 layers) with a Si topmost layer to limit oxidation. The dashed curve is the optimized reflectivity resulting from the genetic algorithm. The measured reflectivity (points) is much less uniform than what is expected from the design and is shifted to shorter wavelengths than would be expected due to contraction. The solid curve in figure 


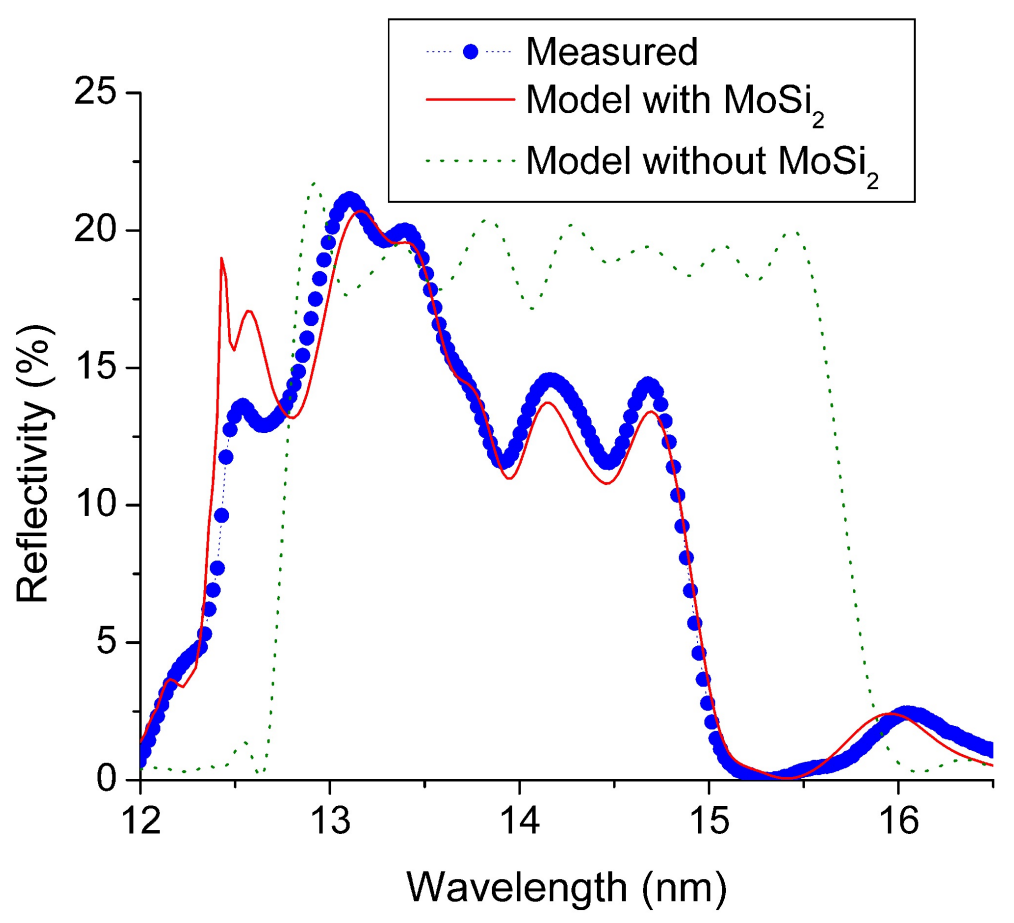

Figure 2.10: This graph shows an optimization for a 40 bi-layer, 45-degree Mo/Si mirror that did not include $\mathrm{MoSi}_{2}$ formation (dashed black curve) and the same multilayer stack with silicide formation (solid red curve). We deposited this mirror and measured it (blue curve). Notice the good agreement to the model that included silicide.

2.10 is calculated by including the silicide layers in the modeled structure and is in very good agreement with the measured reflectivity. The main area of disagreement is near the $\mathrm{Si} \mathrm{L}$ edge at $12.4 \mathrm{~nm}$ and could be a result of inaccuracy of the $\mathrm{MoSi}_{2}$ optical constants.

Figure 2.11 shows the results for a broadband mirror that incorporated silicide in its optimization. This mirror was composed of 50 bi-layers (100 layers) with Si being the topmost layer to limit oxidation. The structure of this multilayer is shown in figure 


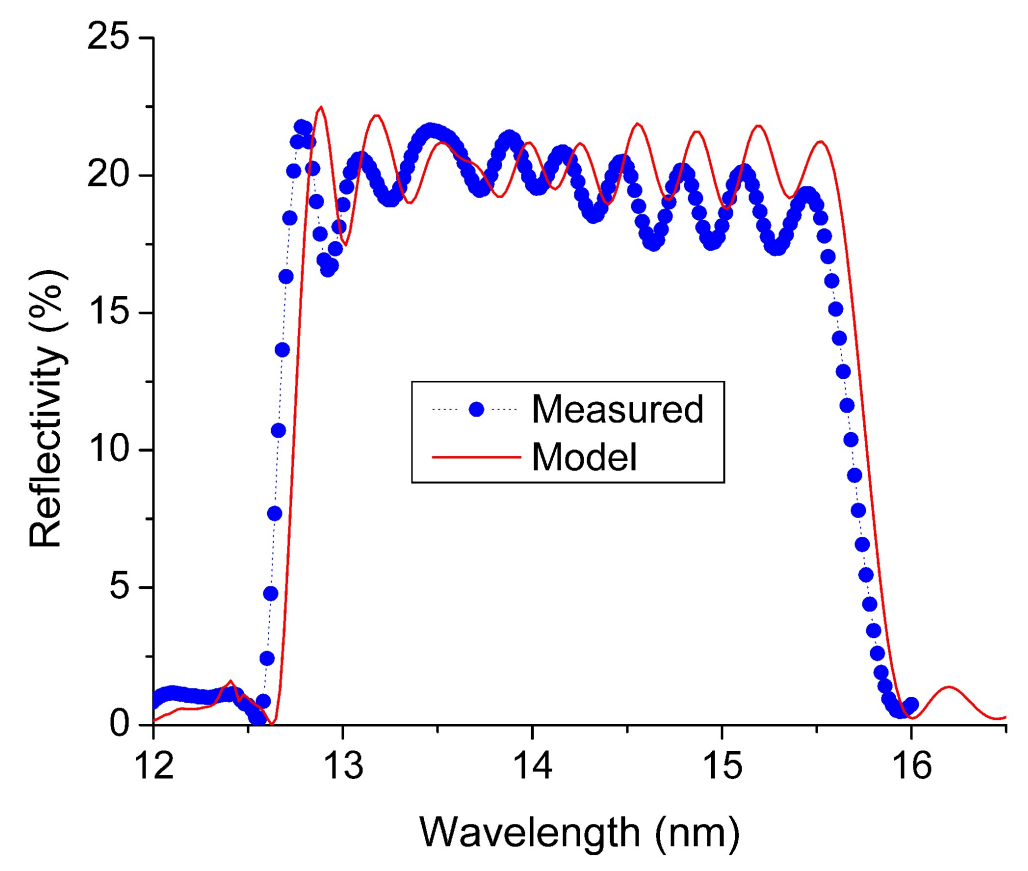

Figure 2.11: This graph shows the reflectivity for the 50 bi-layer Mo/Si mirror when the model with $\mathrm{MoSi}_{2}$ is considered. The mirror is a 45 degree mirror with a $3 \mathrm{~nm}$ bandwidth. A cross-section TEM image of this mirror is shown in 2.9.

2.9. Note the good agreement of the measurements with the simulated structure in figure 2.9 and with the design reflectivity in figure 2.11. The agreement is not exact, and as the TEM image shows there are slight discrepancies in the thickness profile (figure 2.9) due to our sputtering system. We believe this accounts for the discrepancies in reflectivity and the slight wavelength shift.

When creating aperiodic multilayers, attention to the exact sputtering rates is critical. For a typical periodic multilayer mirror a $1 \%$ error in the sputtering rate of one of the materials shifts the wavelength of the reflectivity curve by a factor of 
$\gamma^{*} 1 \%$ or $(1-\gamma)^{*} 1 \%$. For an aperiodic multilayer an error in the deposition rate of one of the materials not only shifts the reflectivity curve but will also change the shape. Furthermore, if we consider a layer of Mo in an aperiodic multilayer that is close to the critical thickness then a shift in sputtering rates could change which side of the critical thickness the layer is on, changing the $\mathrm{MoSi}_{2}$ thickness by $15 \%$ and a $7 \%$ change in Mo thickness. In an aperiodic multilayer these changes will not only shift the reflectivity curve but also change the shape of the reflectivity curve because these changes will only occur in a few layers. If the simulation is to be realistic, care must be taken to insure that the Mo thickness for each layer determined by the optimization is further away from $2 \mathrm{~nm}$ than the thickness error of the sputtering system.

In the following section we will consider an alternative method designed to operate at lower photon energies (longer wavelength), where Mo/Si has poor reflectivity.

\subsection{Tri-Material Multilayers for Longer Wavelengths}

\subsubsection{Why Three Materials Can Be Better Than Two}

In the previous section we considered the use of aperiodic multilayers to shape femtosecond/attosecond pulses. Mo/Si multilayers were selected because the optical constants work well in the energy range of 70-100 eV. However, as seen in figure 1.11 HHG radiation also works well below $70 \mathrm{eV}$, meaning other material combinations are required. Most material combinations do not obtain the high reflectivity of Mo/Si. 
Improving the reflectivity and bandwidth for multilayers at longer wavelengths is critical to femtosecond/attosecond HHG sources.

As stated in 1.2.3 the selection of materials used in a multilayer optic depends on the photon energy or wavelength that it will be used at. Specifically, in the wavelength range of 25 to $50 \mathrm{~nm}$ (50 to $25 \mathrm{eV}$ ) multilayers are used as focusing and imaging elements for research in high harmonic femtosecond chemistry[62] and solar astrophysics[10] imaging such as the He II $30.4 \mathrm{~nm}$ line. Currently, the highest published results on reflectivity in this region are obtained with two-materials magnesium / silicon carbide $(\mathrm{Mg} / \mathrm{SiC})$ multilayers and are around $40 \%$ to $50 \%[63]$.

The relatively high reflectivity at these long wavelengths comes with a drawback of reduced bandwidth. In femtosecond/attosecond applications bandwidth is very important as a consequence of equation (2.9). Integrated reflectivity is more desirable as a merit function for mirror performance than the peak reflectivity. For example, the odd harmonics of a Ti-sapphire laser high harmonic generation are separated by $2 \mathrm{~nm}$ in the vicinity of the $27^{\text {th }}$ harmonic $(30 \mathrm{~nm}$ or $42 \mathrm{eV}$ ). The typical bandwidth of an optically thick (highest obtainable reflectivity) $\mathrm{Mg} / \mathrm{SiC}$ multilayer is $2.9 \mathrm{~nm}$, limiting their use to a single harmonic. Which is fine for certain single harmonic applications, but the limited bandwidth produces longer pulse durations.

Several methods have been implemented to increase the bandwidth of multilayer coatings. The first method to increase bandwidth is to use fewer periods in the multilayer stack. Multilayers are artificial Bragg crystals; they use temporal coherence 
to obtain high reflectivity. In other words, each reflective interface adds coherently to the one above it. As stated in the introduction, for an optically thin stack, the temporal/longitude coherence length must be equal to the thickness of the multilayer:

$$
L_{c o h}=\frac{\lambda^{2}}{2 \Delta \lambda} \approx d \cdot N \Rightarrow \Delta \lambda \propto N^{-1}
$$

where $\mathrm{L}_{c o h}$ is the coherence length, $\lambda$ is the center wavelength, $\Delta \lambda$ is the bandwidth, $\mathrm{d}$ is the optical depth (d-spacing) of the multilayer, and $\mathrm{N}$ is the number of periods of thickness d. However, using few bi-layers implies lower peak reflectivity, as the peak reflectivity scales as $\mathrm{N}^{2}$.

The second method implemented is to make aperiodic multilayers[61][64] as discussed in section 2.3. This method also trades off peak reflectivity for larger bandwidth; however, the integrated reflectivity is often higher than achievable by reducing the number of layers. However, the aperiodic structure could introduce nonuniform phase response and affect the temporal structure of the reflected radiation. Although, this provides a mechanism for pulse compression[53], in general the use of aperiodic optics with high harmonic sources in femtosecond dynamic studies requires full characterization of the source, as well as the optics, to insure that the femtosecond pulses are not inadvertently broadened.

An alternative solution that has been used at longer wavelength $(>50 \mathrm{~nm})[65][66]$ is to use more than two materials in each repeating period of the stack. This allows for higher reflectivity per period in the multilayer stack. Thus higher reflectivity is obtained in fewer periods, therefore increasing the bandwidth while maintaining high 
reflectivity. Three material multilayers have also been proposed and measured for use in both the extreme ultraviolet and the soft x-ray regime of the spectrum[67][68]. At 25-50 nm, previous research using tri-material multilayers did not demonstrate improvement over standard bilayer $\mathrm{Mg} / \mathrm{SiC}$ due to the materials chosen in the study[67]. In the next subsection, we report the design and fabrication of a tri-material multilayer that shows good improvements on both the reflectivity and bandwidth.

\subsubsection{Material Selection, Optimization, and Fabrication of Tri-Material Coatings}

The three materials chosen for improving multilayer bandwidth and maintaining high reflectivity in the 25 to $50 \mathrm{eV}$ photon energy range (25 to $50 \mathrm{~nm}$ ), were $\mathrm{Mg}$, $\mathrm{SiC}$, and $\mathrm{Sc} . \mathrm{Mg}$ was chosen as the low $\mathrm{Z}$ element, because the $\mathrm{L}_{3}$ absorption edge is located at $49.5 \mathrm{eV}(25.0 \mathrm{~nm})$. This makes $\mathrm{Mg}$ optically transparent $(\beta \approx 0)$, and it is typically used as a spacer for multilayers in this wavelength range. $\mathrm{SiC}$ is usually chosen as the high $\mathrm{Z}$ compound (absorber) in $\mathrm{Mg}$ based multilayers due to its low interdiffusion and interface roughness. In this work, the author chose scandium to be a third high $\mathrm{Z}$ material. Sc was chosen because its $\mathrm{M}_{2,3}$ absorption edge at $28.3 \mathrm{eV}$ $(43.8 \mathrm{~nm})$ makes the real part of its index of refraction greater than $1\left(\delta_{\mathrm{Sc}}<0\right)$. It is suitable in multilayers between 35 and $50 \mathrm{~nm}[69]$. It is expected that the reflection from the $\mathrm{Sc} / \mathrm{SiC}$ interface is strong because $\delta_{\mathrm{Sc}}<0$ and $\delta_{\mathrm{SiC}}>0$. This is evident 
from the normal incident Fresnel reflectivity:

$$
R_{\perp}=\frac{\left|n_{1}-n_{2}\right|^{2}}{\left|n_{1}+n_{2}\right|^{2}}=\frac{\left(\delta_{2}-\delta_{1}\right)^{2}+\left(\beta_{1}-\beta_{2}\right)^{2}}{\left(2-\delta_{1}-\delta_{2}\right)^{2}+\left(\beta_{1}+\beta_{2}\right)^{2}} \approx \frac{\left(\delta_{2}-\delta_{1}\right)^{2}+\left(\beta_{1}-\beta_{2}\right)^{2}}{4}
$$

where $R$ is the reflectivity, $n_{1}$ is the index of refractive for the top material and $n_{2}$ is the refractive index from the bottom material. The optical constants[14][15][16] of the three materials are shown in figure 2.12.

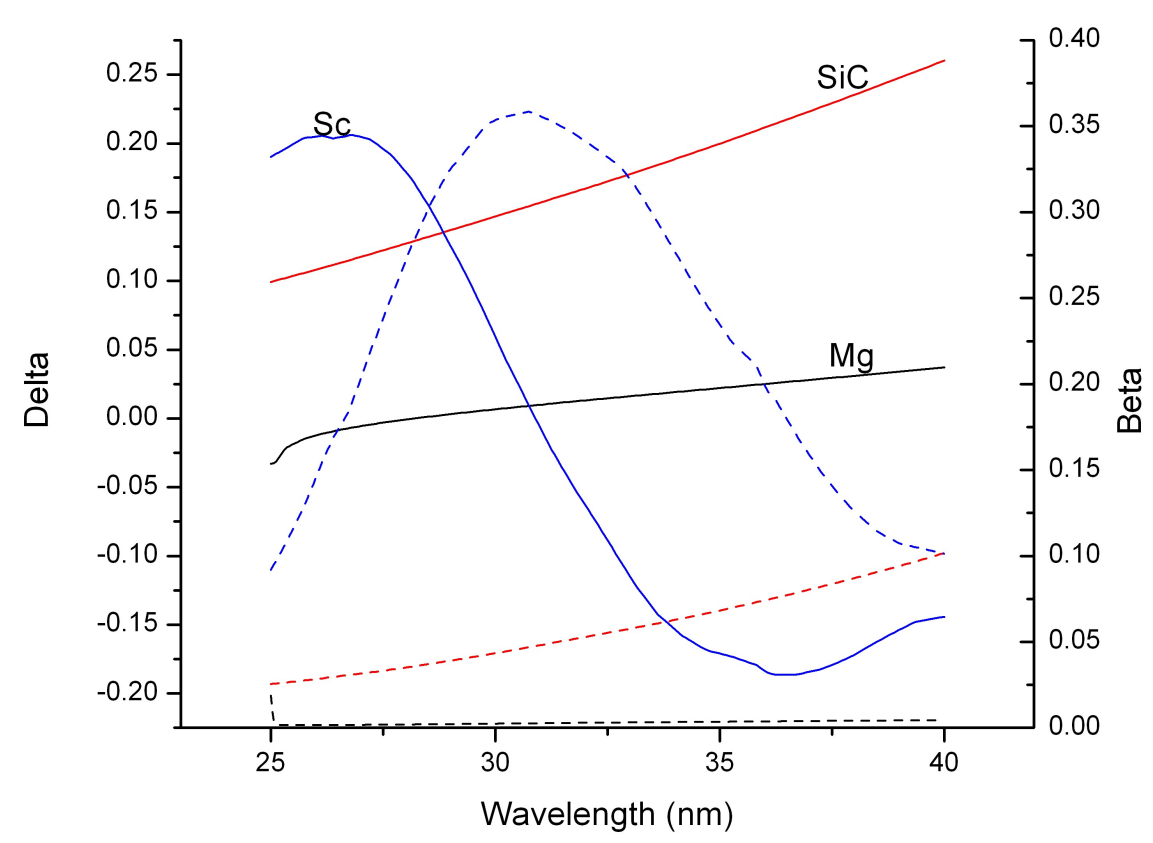

Figure 2.12: This graph shows the optical constants, $\delta$ and $\beta$, for $\mathrm{Mg}$, Sc, and SiC. The solid line represents delta while the dotted lines represent beta. The index of refraction uses the standard convention: $\mathrm{n}=1-\delta+i \beta$.

Four samples were made for this experiment: $2 \mathrm{Mg} / \mathrm{SiC}$ multilayers and $2 \mathrm{Mg} / \mathrm{Sc} / \mathrm{SiC}$. One set $(1 \mathrm{Mg} / \mathrm{SiC}$ and $1 \mathrm{Mg} / \mathrm{Sc} / \mathrm{SiC})$ was designed for a peak reflectivity at $37 \mathrm{~nm}$ near the optimal location for the optical constants. The other set was designed for 
a reflectivity peak at $28 \mathrm{~nm}$ to be away from the optimal difference in optical constants. For the $28 \mathrm{~nm}$ samples, we used non-optically thick stacks for both samples. A brute force reflectivity simulation was performed to optimize the material thickness of each sample. The simulations determined the peak reflectivity for multilayers at the chosen wavelength. The multilayers simulated were for optically thick stacks. As predicted by the theory[66] the order of the materials is critical when three or more materials are used.

For the $37 \mathrm{~nm}$ samples optimal parameters for the $\mathrm{Mg} / \mathrm{Sc} / \mathrm{SiC}$ were: $\mathrm{d}=19 \mathrm{~nm}$, $\gamma_{1}=0.2\left(\gamma_{1}\right.$ is defined as: SiC thickness $\left./ \mathrm{d}\right), \gamma_{2}=0.13\left(\gamma_{2}\right.$ is defined as: Sc thickness / d), $\mathrm{N}=30$ tri-layers. For the $\mathrm{Mg} / \mathrm{SiC}$ sample $\mathrm{d}=19.5 \mathrm{~nm}, \gamma_{1}=0.33, \mathrm{~N}=35$ bi-layers. For the $28 \mathrm{~nm}$ samples optimal parameters for the $\mathrm{Mg} / \mathrm{Sc} / \mathrm{SiC}$ were: $\mathrm{d}=$ $15.4 \mathrm{~nm}, \gamma_{1}=0.2, \gamma_{2}=0.07, \mathrm{~N}=30$ tri-layers. For the $\mathrm{Mg} / \mathrm{SiC}$ sample $\mathrm{d}=14.0$ $\mathrm{nm}, \gamma_{1}=0.3, \mathrm{~N}=40$ bi-layers.

The samples were fabricated on polished silicon wafers using magnetron sputtering. DC sputtering was used for $\mathrm{Mg}$ and Sc targets and regulated by power at 100 W, while RF sputtering was used for $\mathrm{SiC}$, regulated at $275 \mathrm{~W}$. The samples were fabricated under a 1.0 mTorr argon pressure during the deposition. The top layer of each sample is $\mathrm{SiC}$ to prevent oxidation. 


\subsubsection{Reflectivity Measurements}

The samples were measured at ALS beamline 6.3.2 with a setup that was described in section 1.2.4. The samples were measured at 5 degrees from normal incidence. The reflectivity for the $37 \mathrm{~nm}$ samples is shown in Figure 2.13, while the reflectivity for the $28 \mathrm{~nm}$ samples is shown in figure 2.14 .

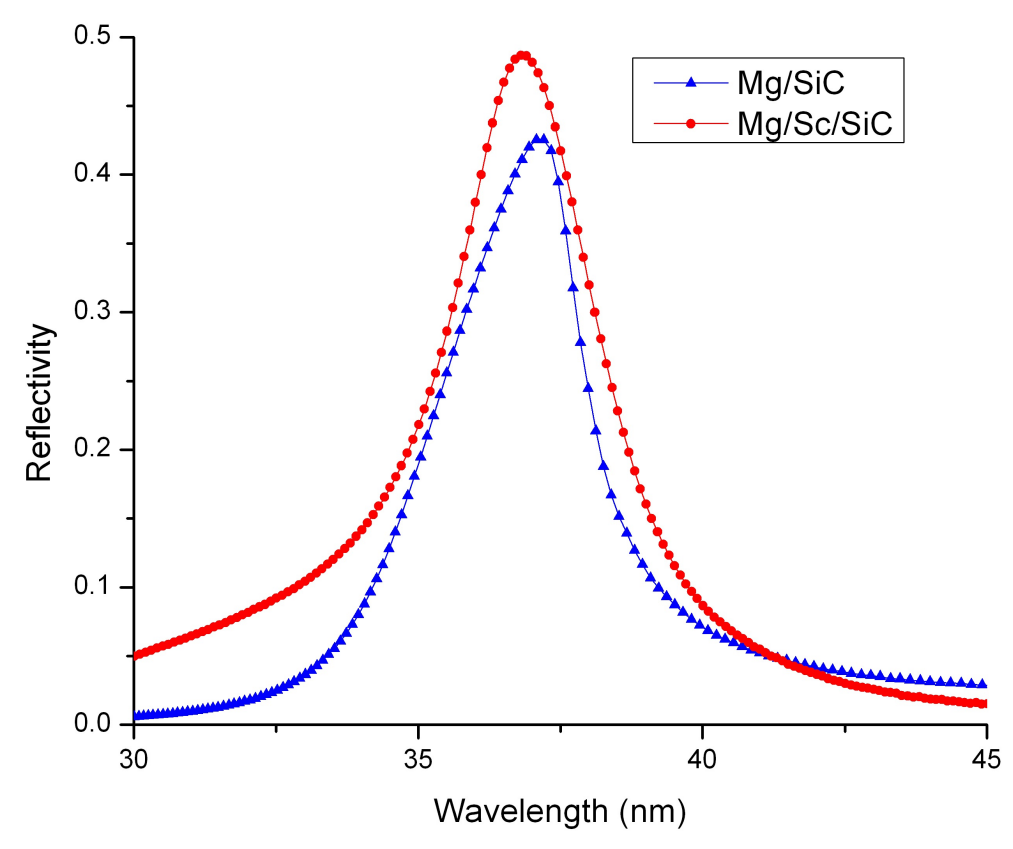

Figure 2.13: The reflectivity of optimized, optically thick $\mathrm{Mg} / \mathrm{SiC}$ and $\mathrm{Mg} / \mathrm{Sc} / \mathrm{SiC}$ multilayers is shown for the samples optimized for $37 \mathrm{~nm}$. The tri-layer multilayer has a FWHM bandwidth of $3.2 \mathrm{~nm}$ and a peak reflectivity of $48.7 \%$ at $36.8 \mathrm{~nm}$ (parameters for tri-layer: $\mathrm{Mg} / \mathrm{Sc} / \mathrm{SiC}=12.73 / 2.47 / 3.80 \mathrm{~nm} ; \mathrm{N}=30$ ). The bi-layer $\mathrm{Mg} / \mathrm{SiC}$ has a FWHM bandwidth of $3 \mathrm{~nm}$ and a peak reflectivity of $42.5 \%$ at 37.1 $\mathrm{nm}$ (parameters for bi-layer: $\mathrm{Mg} / \mathrm{SiC}=13.1 / 6.4 \mathrm{~nm} ; \mathrm{N}=35$ ).

As shown in figures 2.13 and 2.14, the reflectivity of the tri-material multilayer is equally high at producing high reflectivity as the $\mathrm{Mg} / \mathrm{SiC}$ multilayers. In figure 2.13 
the reflectivity of the optimized tri-material stack is even higher than the optimized bi-material stack. Because the tri-material stack uses fewer periods, the intergraded reflectivity and the bandwidth are larger.

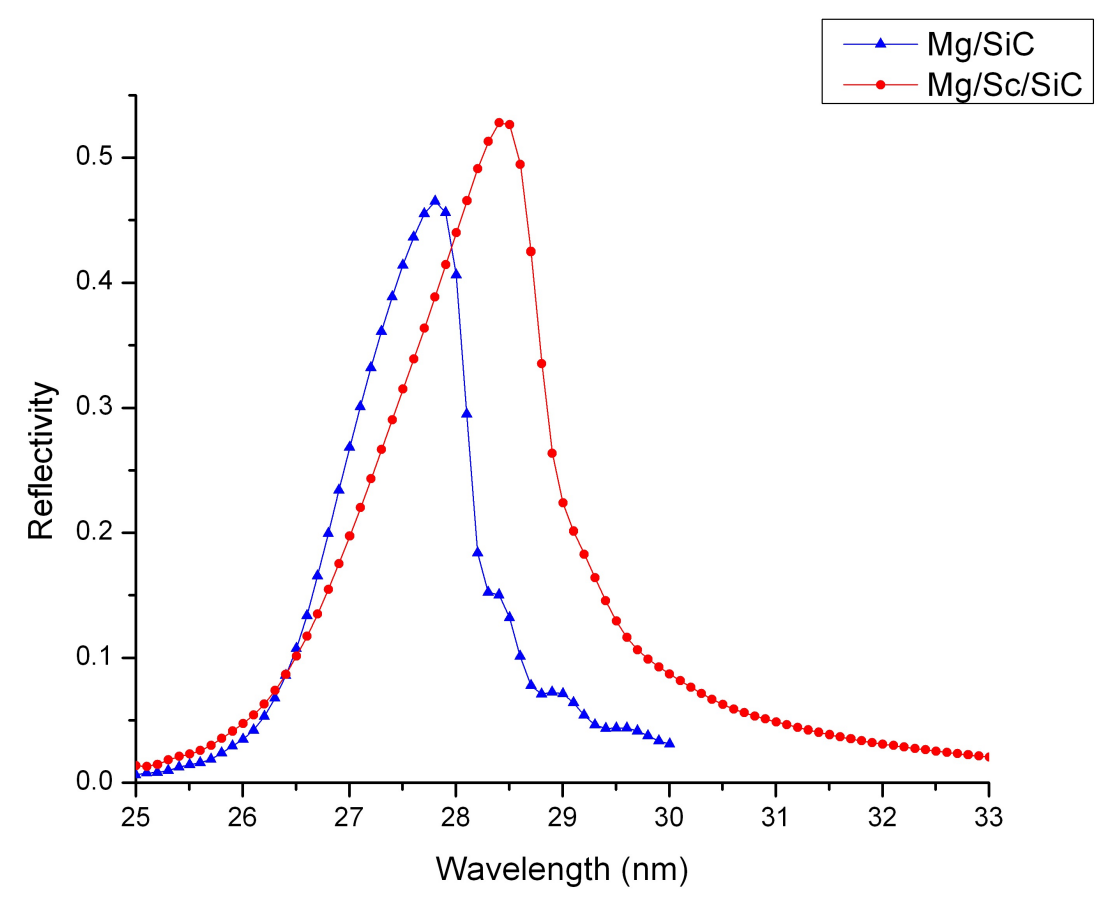

Figure 2.14: The reflectivity of optimized, 30 period $\mathrm{Mg} / \mathrm{SiC}$ and $\mathrm{Mg} / \mathrm{Sc} / \mathrm{SiC}$ multilayers is shown for the samples optimized for $28 \mathrm{~nm}$. The tri-layer multilayer has a FWHM bandwidth of $1.6 \mathrm{~nm}$ and a peak reflectivity of $52.8 \%$ at $28.4 \mathrm{~nm}$ (parameters for tri-layer: $\mathrm{Mg} / \mathrm{Sc} / \mathrm{SiC}=11.24 / 1.08 / 3.08 \mathrm{~nm} ; \mathrm{N}=30$ ). The bi-layer $\mathrm{Mg} / \mathrm{SiC}$ has a FWHM bandwidth of $1.2 \mathrm{~nm}$ and a peak reflectivity of $46.5 \%$ at $27.8 \mathrm{~nm}$ (parameters for bi-layer: $\mathrm{Mg} / \mathrm{SiC}=9.8 / 4.2 \mathrm{~nm} ; \mathrm{N}=40$ ). 


\subsection{Phase \& Quarter-Wave Plate Multilayers for Femtosecond/Attosecond Applications}

With femtosecond/attosecond light sources, often times it is useful to have control over the polarization of the light being produced. High harmonic radiation is linearly polarized due to the nature of the three step process used to produce it as described in section 1.3.3. Often it is of interest to produce radiation that is not linearly polarized as certain experiments require elliptical or circularly polarized radiation. For example the magneto-optical experiments described in chapter 4 require circularly polarized EUV radiation. At visible wavelengths either total internal reflection from a Fresnel rhomb or a doubly refracting crystal is used to convert linearly polarized radiation to circularly polarized radiation. Neither option is possible in the EUV, as no material is doubly refracting and the phase shift from total external refraction is so small that many mirrors would be required to create a device similar to a Fresnel rhomb. However, by utilizing multilayer optics it is possible to convert linearly polarized radiation into circularly polarized radiation. The multilayer will be used not in reflection but in transmission, and it will be used at the Brewster's angle.

\subsubsection{Brewster Angle Transmission Multilayers}

Before calculating the phase shift of a transmission multilayer, the author will explain how a phase shift can form linear to elliptical polarization upon transmission 
through a material. Consider a piece of dielectric material with a thickness, d, and index of refraction, $\mathrm{n}$, with light incident on its surface at an angle $\theta$ as in figure 2.15. One can ask what is the transmission of the light through the material for the transverse electric and transverse magnetic fields. This problem can be solved using boundary conditions ( 4 equations and 4 unknowns with respect to the incident field for each polarization). The boundary values can also be solved pictorially by using ray tracing. In this example, it is more instructive to use ray tracing to solve the boundary conditions. The transmitted electric field is given by summing all the rays that transmit through the material. For example, a part of the light will be reflected from the first surface, while a part will be transmitted. Part of this transmitted light will be transmitted through the back interface and part reflected. This second reflected part will be propagated through the material again and with part reflecting and part transmitting and so fourth and so fourth. Mathematically, the transmitted electric field can be written:

$$
E_{T}=E_{0} t^{2} e^{i \varphi}+E_{0} t^{2} r^{2} e^{i 2 \varphi}+E_{0} t^{2} r^{4} e^{i 3 \varphi}+\cdots
$$

where $r$ is the reflection Fresnel coefficient, $t$ is the transmission Fresnel coefficient, and $\varphi=\frac{4 \pi}{\lambda} n d \cos \theta$ is the phase shift due to propagation. Equation 2.21 is a geometric series and can be solved:

$$
E_{T}=\frac{E_{0} t^{2} e^{i \varphi}}{1-r^{2} e^{i \varphi}}=\frac{E_{0} t^{2}\left(e^{i \varphi}-r^{2}\right)}{1-r^{2} \cos \varphi+r^{4}}
$$

Now suppose that the light is incident at Brewster's angle. In this case, the denom- 
inator of equation 2.22 becomes $1(r=0)$ and the $t$ in the numerator also becomes 1 , but only in the transverse magnetic or p-polarization case. For the transverse electric or s-polarization case these two terms are still included. This gives rise to a difference in transmitted amplitude, and more importantly, a relative phase difference between the incident s-polarized and p-polarized radiation. This Brewster's angle phase difference, in transmission occurs because the complex phase term $e^{i \varphi}-r^{2}$ in equation (2.22) differs for s-polarization and p-polarization. If the incident radiation was linearly polarized at an angle so it contained both s-polarization and p-polarization, then the resulting transmitted radiation through the material (at Brewster's angle) would be elliptically polarized.

If a phase difference of $90^{\circ}$ is achieved and the intensity of transmitted s-polarized and p-polarized radiation are equal, then the light becomes circularly polarized. A mirror such as this is ofter refered to as a quarter wave $\left(\frac{\lambda}{4}\right)$ plate. Because of absorption at EUV wavelengths a single material will be able produce a $90^{\circ}$ phase shift. However, multilayers used in transmission can produce circularly polarized EUV radiation[70][71]. Each layer in the multilayer acts coherently with every other layer to obtain a $90^{\circ}$ phase shift.

\subsubsection{Polarization Measurements}

To determine the usability of EUV quarter-wave plates the authors chose to fabricate optimized periodic Mo/Si multilayers for use in the first test. The optimization 


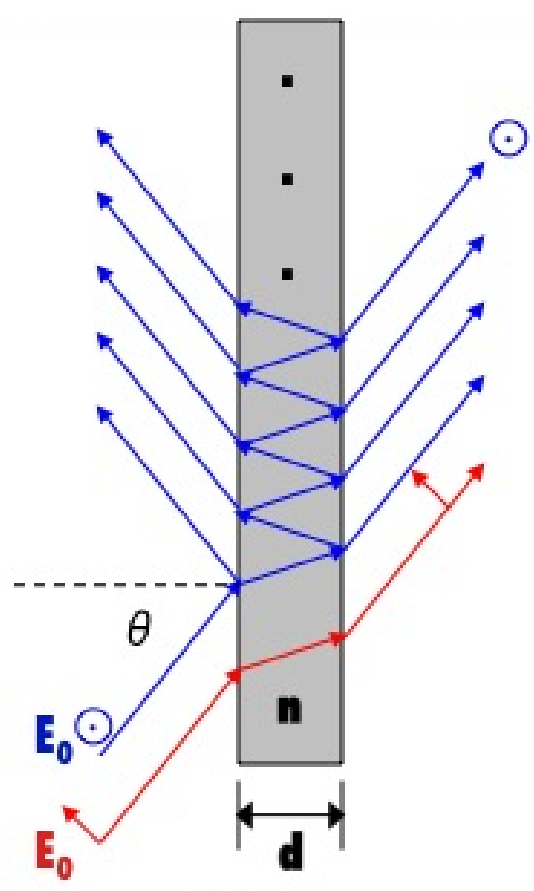

Figure 2.15: The transmission of light through a dielectric material with index $n$, thickness $d$, and angle $\theta$. The blue line shows s-polarization at the Brewster's angle while the red shows p-polarization.

was to maximize transmission efficiency of the polarizer while maintaining a $90^{\circ}$ phase shift. A periodic multilayer was chosen since the goal was to test the efficiency at a specific wavelength. An aperiodic multilayer could have been chosen to increase the usable bandwidth range, but the magnetic application was to utilize an isolated harmonic from a HHG source. Thus, this limitied the necessity to create a polarizer that operated over a larger bandwidth. The optimization used a three parameter brute force algorithm of d-spacing, $\gamma$, and multilayer angle ${ }^{7}$. Multilayer angle was chosen as a parameter because the Brewster's angle of a material in the EUV is[1]:

\footnotetext{
${ }^{7} \mathrm{MoSi}_{2}$ interfaces described in section 2.3 .1 are used in the simulations.
} 
$\phi_{B} \simeq \frac{\Pi}{4}-\frac{\delta}{2}$ this is not exactly 45 degrees and higher efficiency can be obtained at slight angles away from $45^{\circ}$.

Samples were fabricated using the DC magnetron sputtering described in section 1.2.4. The Argon pressures and power settings were optimized to allow for low stress film growth. The test multilayer was fabricated on a photoresist coated Si wafer. The photoresist would be etched away after coating leaving a free standing multilayer film. Sputtered Si was used as the first (bottom) and last (top) layer to prevent oxidation. The multilayer coated wafer was measured at the ALS beamline 6.3.2 to check the d-spacing and $\gamma$. After measurements a wafer piece was glued to an aperture, and the photoresist was chemically removed. This allowed for the liftoff of a free standing multilayer film.

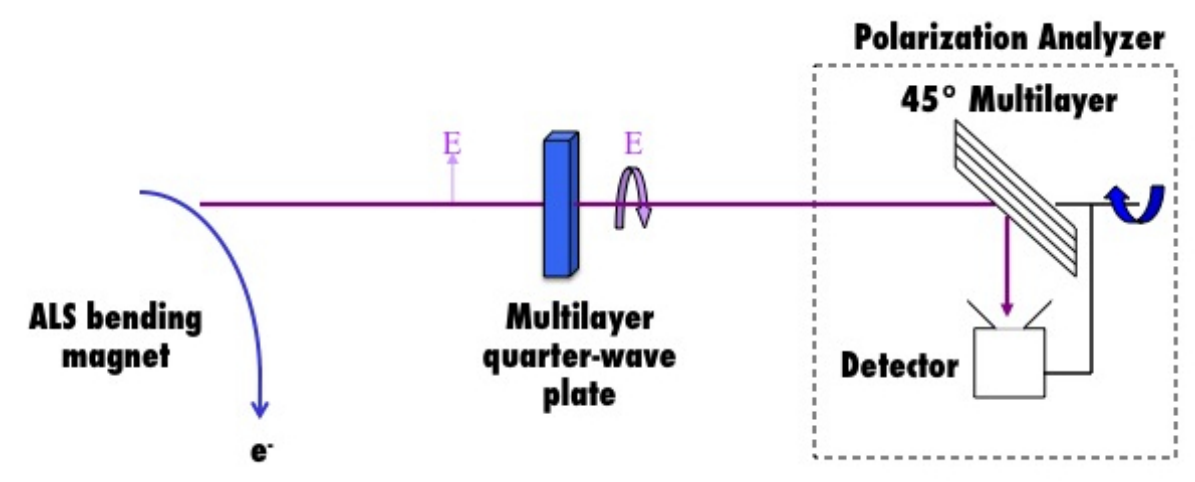

Figure 2.16: The ALS setup for measuring polarization. Linearly polarized EUV from a bending magnet is passed through the multilayer quarter-wave plate. The intensity of the EUV then is measured as a function of rotation angle.

The transmission quarter-wave plate multilayer was measured in the ALS beamline 6.3.2 facility that was described in section 1.2.4. To measure the polarization we used 
a specially designed setup as shown in figure 2.16. Radiation produced in the plane of a bending magnet is linearly polarized[72]. The linearly polarized light then passes through a multilayer quarter-wave plate. The light then reflects off a Brewster angled multilayer and into a detector. The Brewster angle multilayer is at $45^{\circ}$ and only reflects $\mathrm{S}$ polarized light. The analyzer is then rotated to determine the intensity as a function of angle. The results are shown in figure 2.17. For linearly polarized light one would expect a perfect sine or cosine wave, with peak signal intensity and zero signal intensity $90^{\circ}$ out of phase as seen in the figure. For circularly polarized light the polarization analyzer should be independent of detector angle. All other polarization states (elliptically polarized radiation) will have curves with slight modulation.

The multilayer quarter-wave plate has two defined angles. There is the angle with respect to direction of the propagation of the EUV and the multilayer, this will be at the Brewster's angle for this angle. There is also the azimuthal angle of rotation around the propagating radiation. If the $\mathrm{S}$ and $\mathrm{P}$ transmissions were identical then this angle would be half way between $\mathrm{S}$ and $\mathrm{P}\left(45^{\circ}\right)$. This angle is optimized to the transmission efficiencies of the $\mathrm{S}$ and $\mathrm{P}$ polarization via: $\theta_{a z}=\arctan \frac{T_{s}}{T_{p}}$. If this angle is not correct then deviations from circular polarization will occur. For a full discussion using Jones matrixes see appendix B.

Along with measuring the reflectivity and polarization of a multilayer coating, it is critical to measure the reflected phase of the multilayer. The reflectivity and the reflected phase determines the effect the optic will have on the pulse shape. In the 
Mo/Si 1/4 Waveplate (@13.5 nm)

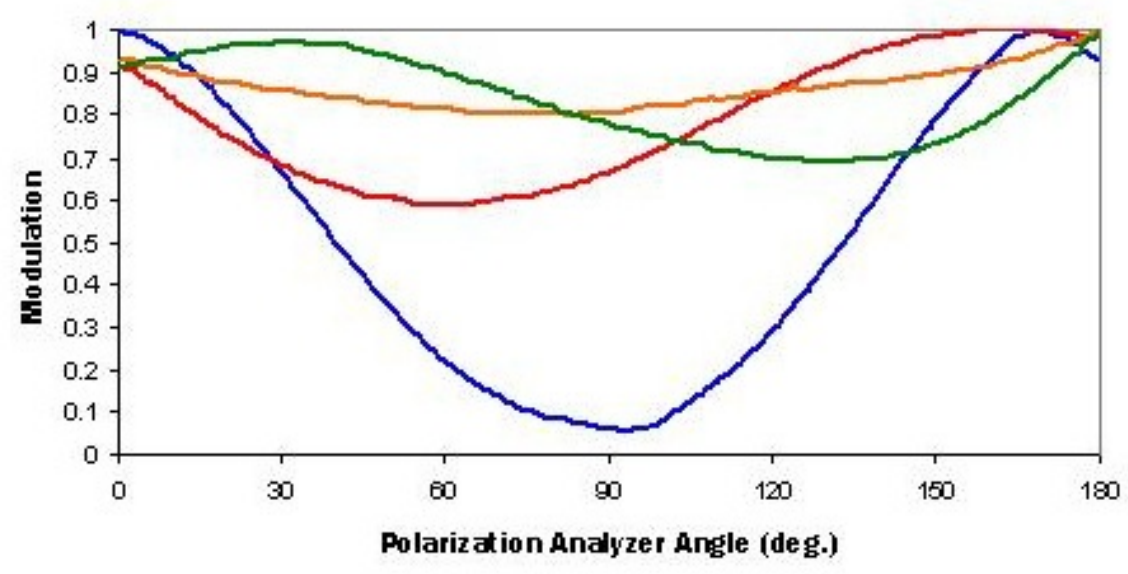

- No ML maneplate _30deg ML maveplate _-35deg ML maveplate —40deg ML maveplate

Figure 2.17: The graph shows the detector signal as a function the polarizer angle. Notice the ALS (blue curve) is linear and has full modulation at $90^{\circ}$. The orange curve shows the polarization is close to circular (no modulation with polarizer angle). The red and green curves demonstrate the effect of an offset in the azimuthal angle.

following chapter the author will discuss a method to measure the reflected phase of a multilayer optic. He will also discuss the measurement of attosecond pulses. 


\section{Chapter 3}

\section{Pulse Compression \& Attosecond}

\section{Research}

\subsection{Phase Measurements of EUV Multilayer Op- tics}

In the previous chapter, optics to shape and control femtosecond/attosecond EUV pulses were discussed. Also discussed were the effects of GDD and phase of the optics and pulses. Along with the developments of optics, it is also critical to develop metrologies to test and characterize the optics. In this section, a technique is described that allows for the characterization of the reflected phase of a multilayer optic. This enables the determination of how the multilayer optic will effect an attosecond pulse. 


\subsubsection{The Necessity of Phase Measurements}

The development of high harmonic generation (HHG) sources has created a probe for the attosecond world in the EUV spectrum[73][74]. Producing attosecond light pulses requires a light source with a broad bandwidth. As derived in section 2.1.1 FWHM bandwidth for a Gaussian pulse is described by the Fourier transform theory relation between the time domain and the frequency/energy domain:

$$
[\Delta E \Delta \tau]_{F W H M} \geq 1.825 \mathrm{eV} \cdot \mathrm{fs}
$$

where $\Delta \tau$ is the duration of the pulse in fs, and $\Delta E$ is the FWHM bandwidth in $\mathrm{eV}$. For a $\Delta \tau=100$ attosecond pulse, equation (2.9) tells us that the minimum FWHM reflectivity bandwidth $\Delta E$ for the optic needs to be $18 \mathrm{eV}$ without inadvertently broadening the pulse. As described in section 2.2.2, broad bandwidth is not the only requirement to make an ultrashort pulse. To obtain the minimum pulse width, there is a requirement on the phase alignment of every frequency in the pulse. More specifically, the second and higher order term in a Taylor expansion of the phase, with respect to frequency, must be zero:

$$
\phi(\omega)=\phi\left(\omega_{\text {cent }}\right)+\phi^{\prime}\left(\omega_{\text {cent }}\right)\left(\omega-\omega_{\text {cent }}\right)+\phi^{\prime \prime}\left(\omega_{\text {cent }}\right) \frac{\left(\omega-\omega_{\text {cent }}\right)^{2}}{2 !}+\cdots
$$

where $\phi$ is the phase, $\phi\left(\omega_{\text {cent }}\right)$ is the CEP (constant), $\phi^{\prime}\left(\omega_{\text {cent }}\right)$ is the GD (first derivative of the phase), and $\phi^{\prime \prime}\left(\omega_{\text {cent }}\right)$ is the GDD (second derivative of the phase). Recall from section 2.2.2, the term called GDD, or chirp of the pulse, adversely affects the 
pulse size by extending it to a duration of

$$
\tau=\tau_{0} \sqrt{1+\frac{16(\ln 2)^{2}\left\{\phi^{\prime \prime}\left(\omega_{\text {cent }}\right)\right\}^{2}}{\tau_{0}^{4}}}
$$

where $\tau$ is the pulse size, $\tau_{0}$ is the band-width limited pulse duration, and $\phi^{\prime \prime}\left(\omega_{\text {cent }}\right)$ is the GDD evaluated at the central frequency $\omega_{\text {cent }}$ of the pulse's bandwidth. As mentioned in the introduction chapter (section 1.3.4) HHG attosecond pulses are chirped. Because of this every multilayer optic for attosecond pulses must take the reflected phase of the optic into consideration.

To demonstrate the importance of reflected phase measurements of a multilayer, figure 3.1 shows the simulation of a negatively chirped pulse before it is reflected off the positive GDD mirror (figure 3.4) and after the reflection. The reduction in FWHM pulse size is $\sim 50 \%$. For this reason, EUV multilayer optics have been proposed and implemented as optical elements for attosecond HHG pulses[75]. They have also been proposed to compensate for the phase (intrinsic chirp) in the HHG systems[52]. However, no straightforward method has been available to measure the reflected phase of the multilayer as compared to the incident phase. The author's research has developed and implemented a simple method to measure the reflected phase of an EUV multilayer mirror using measurements of the total electron yield (TEY) to probe the standing wave at the mirror surface. 


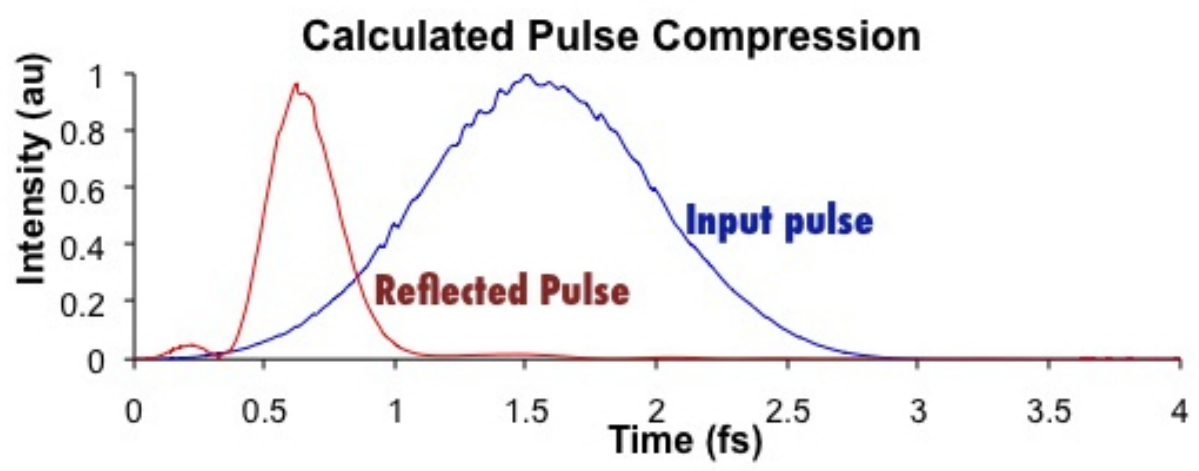

Figure 3.1: An EUV pulse before and after a positively chirped multilayer mirror. The input pulse had a chirp that would exactly be canceled by the multilayer mirror. The incident pulse is assumed to have a gaussian spectrum centered at $90 \mathrm{eV}$ and a negative chirp of $-0.0956 \mathrm{fs}^{2}$.

\subsubsection{Total Electron Yield for Phase Measurement}

Traditional methods for measuring the reflective phase of an optic use interferometric techniques[43]. However, in the EUV these techniques are often limited to grazing incidence or a narrow bandwidth due to the use of a multilayer beamsplitter for normal incidence measurements. Either way, these techniques are difficult to apply due to the stringent constraints, which scale with the wavelength, on position accuracy to obtain the optical phase. For spectral interferometric techniques position accuracy of a fraction of the wavelength is often required, in the EUV this translates to a few $\mathrm{nm}$ in position accuracy and vibration stability.

The technique investigated here uses total electron yield (TEY) along with reflectivity measurements to probe the standing wave at the surface of a multilayer film. The TEY is proportional to the intensity of the standing wave field at the surface of 
a thin film[76]. Multilayer thin films work on the principle of temporal coherence, in other words the reflected wave is coherent with respect to the incident wave at the top surface.

$$
\begin{gathered}
\text { TEY }=C(\omega) I(\omega) \\
I \propto|E|^{2}=\left|\left(E_{0}+E_{r e f}\right)\right|^{2} \\
I \propto\left|E_{0}\left(1+r e^{i \Delta \phi}\right)\right|^{2}=\left|E_{0}\right|^{2}\left(1+r^{2}+2 r \cos (\Delta \phi)\right)
\end{gathered}
$$

where $\mathrm{C}$ carries the material dependence of the TEY, $\left|E_{0}\right|^{2}$ is the incident field, $r$ is the wavelength dependent reflection coefficient $\left(|r|^{2}=R\right.$ where $\mathrm{R}$ is the sample reflectivity), and $\Delta \phi$ is the difference in phase between the incident wave and the reflected wave. Due to the non-zero escape depth of electrons the TEY is not just proportional to the surface intensity. It is proportional to the integrated intensity and material composition as a function of depth multiplied by the probability that the electron escapes the surface of the sample. However, correction factors for this effect can be taken into account[77].

In order to test the method, two different quadratically depth-graded ${ }^{1} \mathrm{Mo} / \mathrm{Si}$ multilayers and one periodic Mo/Si multilayer were produced. For a justification of using a quadratically depth-graded multilayer see appendix C. The multilayers were designed to be used at $10^{\circ}$ from normal incidence, at a central wavelength between 13 to $14 \mathrm{~nm}$, and have average GDD values of $0.0956,-0.0488$, and -0.0001

\footnotetext{
${ }^{1}$ The d-spacing, $d$, of a quadratically depth-graded multilayer with respect to layer number, $n$, is: $d[n]=a n^{2}+b$ where $a$ and $b$ are constants.
} 
$\mathrm{fs}^{2}$. Realistic simulations of these multilayer stacks that included interdiffusion[56][64] (see section 2.3.1) and experimentally verified optical constants[78][79][80] were used as comparison to the measured data. A $4 \mathrm{~nm}$ capping layer of sputtered Si was used to insure the electron signal from the TEY only came from the sputtered Si and not from the Mo layer underneath. The thickness of the capping layer was determined by TEY measurements at the $\mathrm{Mo} \mathrm{M}_{2,3}$ edge as seen in figure $3.2^{2}$.

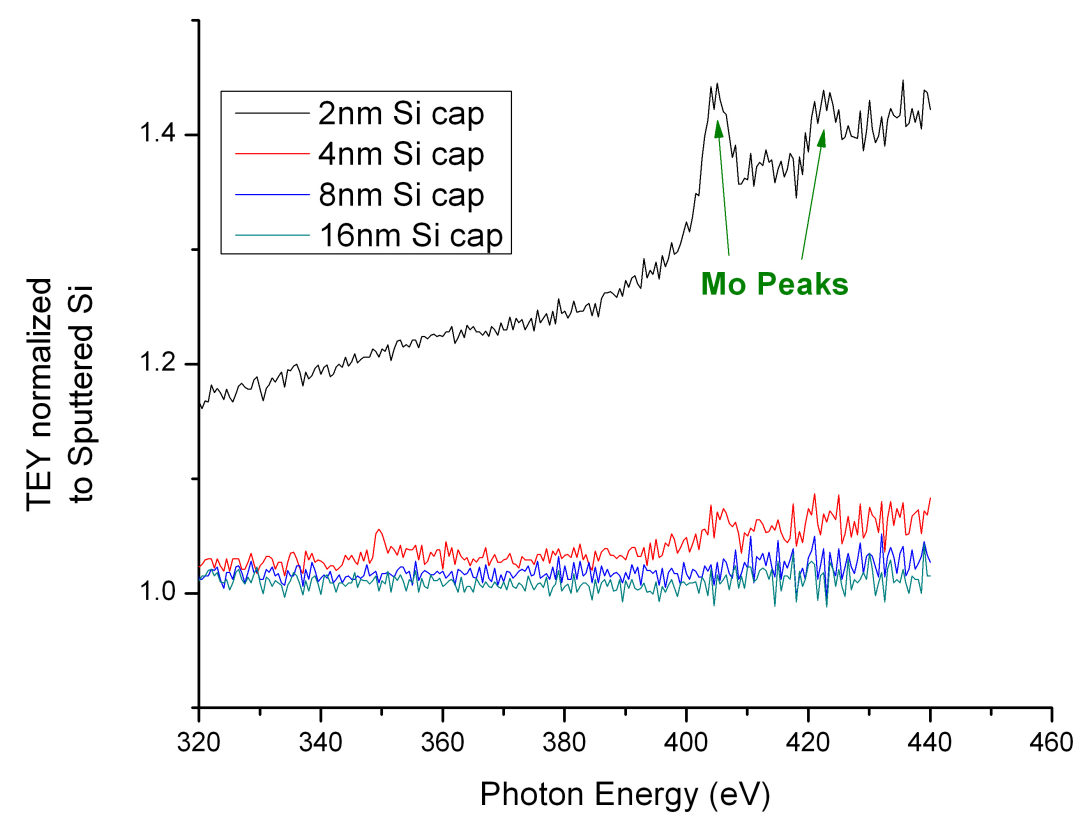

Figure 3.2: This figure shows the normalized TEY measurement of four Mo films with different Si capping layers. As can be seen in the figure, the $2 \mathrm{~nm}$ film is thin enough to allow electrons from the Mo layer beneath to escape the sample. The Mo $\mathrm{M}_{2,3}$ edges at $394 \mathrm{eV}$ and $411 \mathrm{eV}[83]$ are visible. With the $4 \mathrm{~nm}$ Si capping layer, only a slight Mo signal at the edge can be observed.

\footnotetext{
${ }^{2}$ We assume that the electron escape depth is approximately the same for $100 \mathrm{eV}$ as it is for 400 $\mathrm{eV}$, as the inelastic mean free path of an electrons in $\mathrm{Si}$ is only slightly higher at $400 \mathrm{eV}(1.2 \mathrm{~nm})$ than $100 \mathrm{eV}(0.5 \mathrm{~nm})[81][82]$
} 


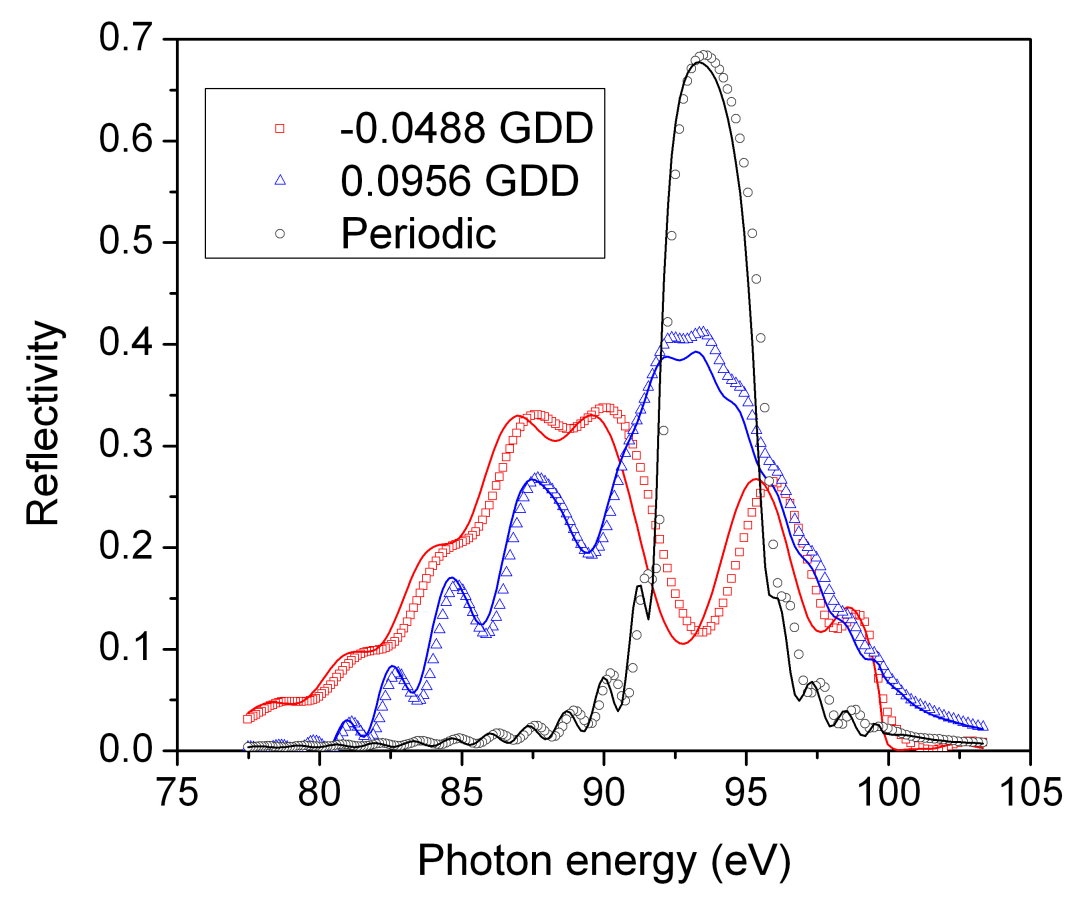

Figure 3.3: Three samples were produced for this experiment: two quadratic depthgraded or chirped samples and one periodic sample. The two quadratic graded samples were designed to have opposite signs for their GDD. The positive GDD sample was designed to have twice the chirp of the negative GDD sample. Plotted are the measured reflectivity curves for the three samples (experimental data points as indicated in the inset) and their simulations (solid curves). The samples are labeled by their GDD.

TEY and reflectivity measurements were performed at ALS beamline 6.3.2[22] which is designed for EUV optical metrology and reflectivity measurements as described in section 1.2.4. The reflectivity measurements are shown in Figure 3.3. The TEY data, shown in Figure 3.4, were collected using a Keithley 428 current amplifier and a collection voltage of $20 \mathrm{~V}$. At $20 \mathrm{~V}$ the current reached $99 \%$ of saturation and the current became virtually independent of the applied voltage. This produced a 


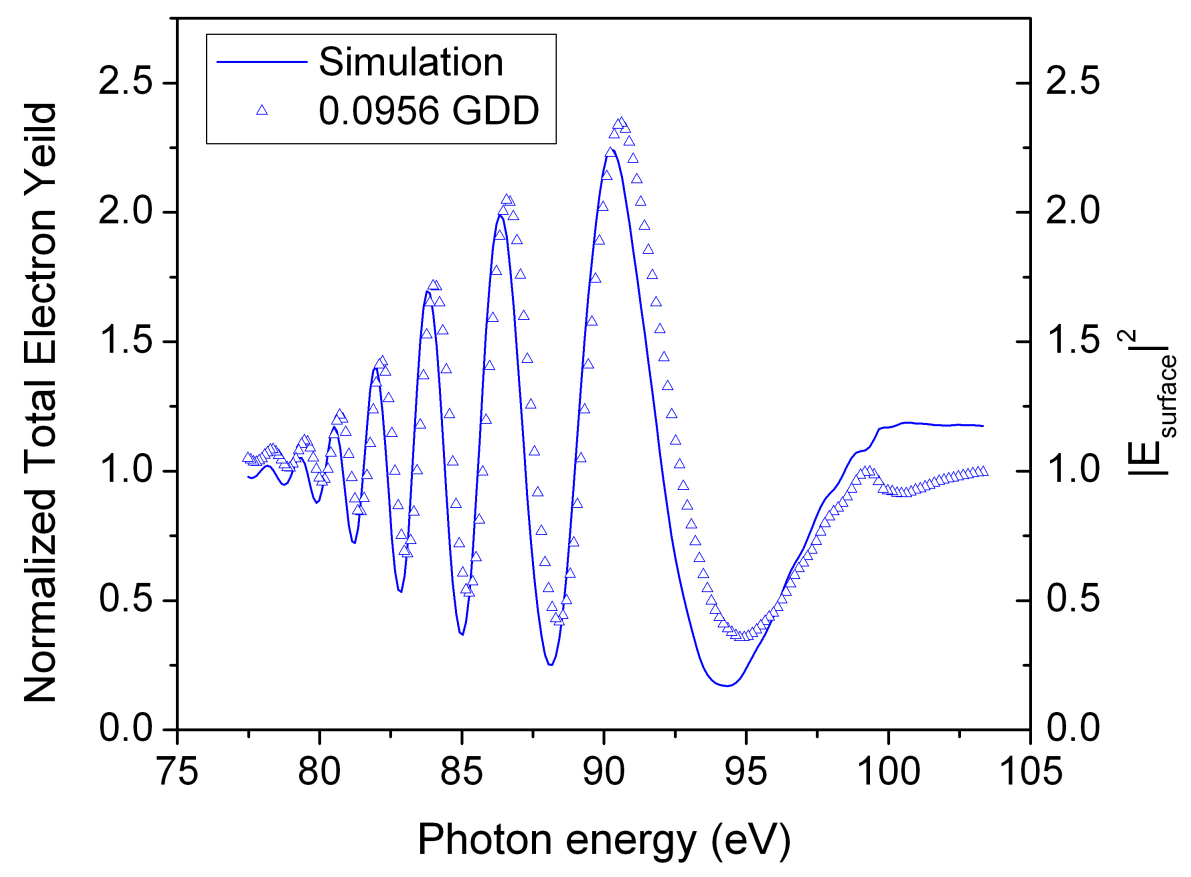

Figure 3.4: Normalized TEY measurements , $\triangle$, shown for the positive GDD sample plotted. The normalization is such that a value of 1 would correspond to the TEY value of sputtered Si. Plotted along the same graph, as a solid curve, is the simulated intensity of the surface electric field based on the modeled reflectivity and the reflected phase.

noise level of less than $\pm 2 \%$. The measured TEY from a $20 \mathrm{~nm}$ thick sputtered Si film was used to normalize out the material dependence $\mathrm{C}(\omega)$ in Equation (3.4) as the top material in the multilayer was sputtered Si. Also, as shown in figure 3.5, the TEY of crystalline $\mathrm{Si}$ is not the same as it is for sputtered Si. This is critical for accurate normalization. The data were also normalized to the ALS storage ring current to account for the different incident field intensities, $E_{0 m l}^{2}$ and $E_{0 \mathrm{Si}}^{2}$, used for the measurements. 
Assuming the normal incidence reflectivity of sputtered Si is negligible:

$$
\frac{\mathrm{TEY}_{m l}}{\mathrm{TEY}_{\mathrm{Si}}}=\frac{C(\omega) E_{0 m l}^{2}\left(1+r^{2}+2 r \cos \Delta \phi\right)}{C(\omega) E_{0 \mathrm{Si}}^{2}}
$$

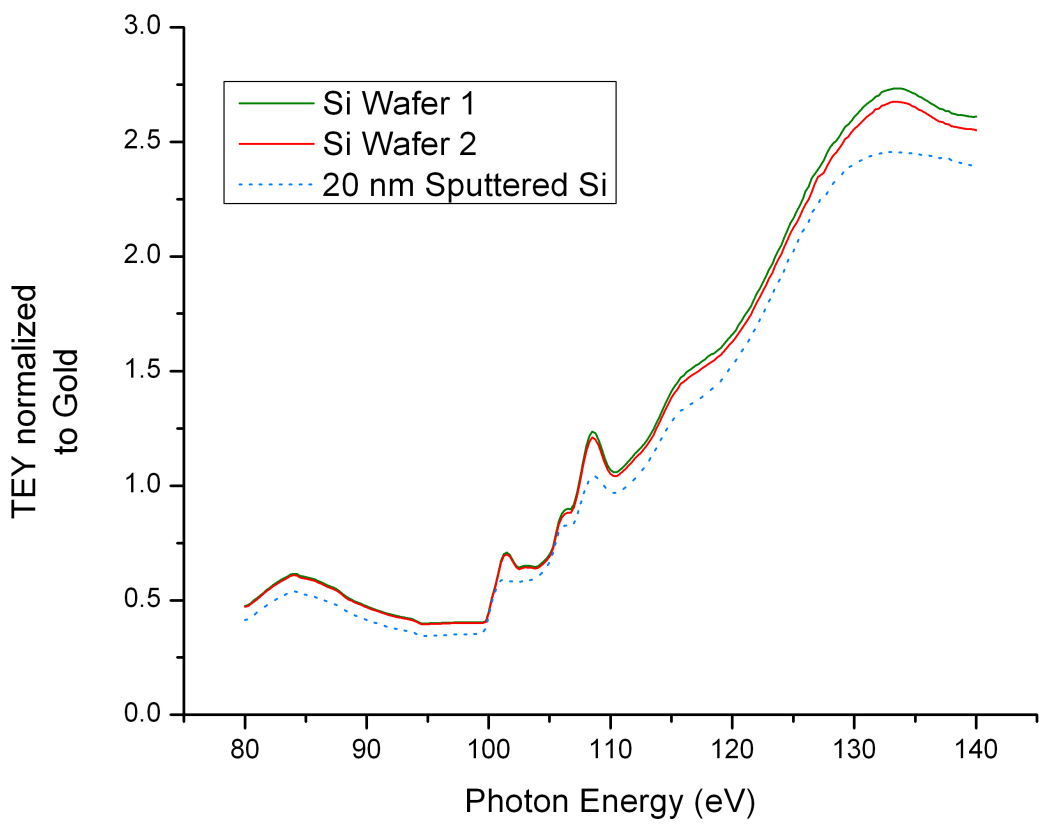

Figure 3.5: This figure shows the measured TEY for crystalline Si wafers and 20 $\mathrm{nm}$ amorphous sputtered Si. The results are normalized to the TEY of gold. Two different $\mathrm{Si}$ wafers were used to demonstrate the accuracy and reproducibility of the measurements.

The phase calculated from the measured reflectivity and normalized TEY is shown in Figure 3.6. By inverting equation (3.4) the difference in reflected phase from the incident phase is retrieved:

$$
\Delta \phi= \pm \cos ^{-1}\left(\frac{J-R-1}{2 \sqrt{R}}\right)+2 \pi n
$$


where $J$ is the normalized photocurrent, $R$ is the square of the reflection coefficient (reflectivity), and $n$ is an integer. As the arccosine function is multivalued, the choice of $n$ and the sign of the arccosine were taken to make $\Delta \phi$ continuous and initialized to have the highest photon energy measured to fall between 0 and $\pi$. It would be difficult to use the second derivative of discrete experimental phase data points to determine the average GDD, because they would appear very noisy. Instead we fit a quadratic polynomial function to the phase and took the second derivative of the polynomial to determine the average GDD. A polynomial fit was chosen to allow the GDD to match the definition given in equation (2.12). The GDD of the three samples was determined to be $0.0962,-0.0439$, and 0.00476 fs 2 , which are in good agreement with the desired delays.

In summary a simple method has been developed to measure the reflected phase of a multilayer with respect to photon energy. This method utilizes the interference at the surface of the optic with the measured total electron yield. Combining the phase measurement with the reflectivity measurement allows one to determine the impulse response and GDD of EUV optics for pulse applications.

\subsection{Attosecond Pulse Measurements}

In a pump/probe measurement knowing the duration of the probe pulse is critical to understanding time constants, constraints on the physics, and dynamics of the system under study. This section describes metrologies for determining the duration 


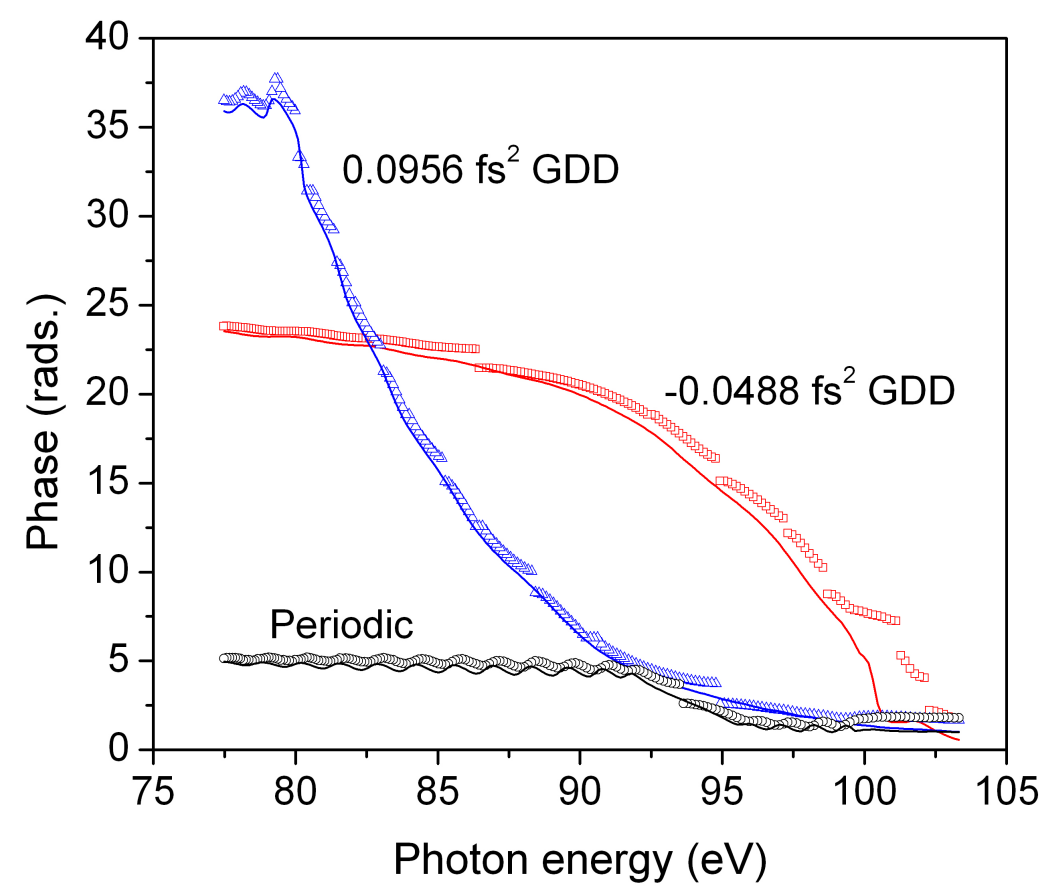

Figure 3.6: Phase for the three samples was reconstructed from the reflectivity and TEY data. Plotted as solid curves are the calculated phases of the multilayers.

of an attosecond pulse. This section includes a description of the experimental setup used to measure the shortest published pulse to date, 80 attoseconds (FWHM).

\subsubsection{Introduction to Ultrafast Pulse Measurements in the Visible and X-ray}

Many techniques in the EUV are derived from pre-existing methods at visible wavelengths or x-rays. A brief review of pulse measurement techniques for visible and x-ray wavelengths will be discussed to provide insight and terminology for the 
discussion of EUV pulse measurements which follow.

When measuring pulses, knowing what information is necessary to measure and fully characterize the pulse dictates the setup of the experiment. As seen in section 2.2.2, it is not sufficient to measure the spectrum alone to determine the pulse duration. Phase information of the second and higher order terms in the Taylor series of the phase is required and is critical in the measurement of any signal. As described, the CEP and GD are not sufficient to determine pulse duration; what is needed is the third and higher order terms in the Taylor series of the phase. An equivalent analogy to reconstructing a pulse from its intensity is the reconstruction of a piece of music knowing only what notes are played, and the relative number of times they are played, but not the order the notes are played in. This would dearly be inadequate. Knowledge of the phase properly places the notes. Similar knowledge is essential for electromagnetic pulse measurements.

In the visible regime, techniques involving nonlinear media are used to measure pulse duration (FWHM of the intensity, $I(t)$ ). The standard method is call intensity autocorrelation[84]. This method uses two identical pulses ,separated in time by a variable delay $\tau$. For example, the two pulses enter into a second harmonic generator (non-linear media), the intensity of the second harmonic is then measured on a slow detector. The intensity autocorrelation signal $S(\tau)=\int I(t) I(t-\tau) \mathrm{d} t$. This signal provides the pulse duration, however all phase information is removed. Meaning that no information on the phase or shape of the pulse can be retrieved. 
More advanced techniques are used to obtain a full retrieval of the pulse shape and duration. Frequency resolved optical gating (FROG)[85][86] and spectral phase interferometry for directed electric-field reconstruction (SPIDER)[87] are the two main methods used in full phase reconstruction. Both methods overlap a reference or gate pulse with the pulse under consideration. The overlap occurs in a nonlinear media producing intensities proportional to the electric field terms. The FROG method will be discussed in more detail as it relates more directly to current attosecond techniques $^{3}$. The FROG uses a gating pulse in a nonlinear element to produce a pulse proportional to the product of the to be measured electric field and a gating function. The gating function originates from the gating pulse; its exact form depends on the nonlinear wave mixing process. This signal is then passed through a spectrometer to resolve the frequency components. Then the gating pulse delay is varied by a small amount of $\tau$. Mathematically this appears:

$$
S(\omega, \tau)=\left|\int_{-\infty}^{\infty} E(t) g(t-\tau) e^{i \omega t} \mathrm{~d} t\right|^{2}
$$

where $E(t)$ is the unknown electric field, $g(t)$ is the gating function of the gate pulse, and $S(\omega, \tau)$ is called a spectrograph function. Notice that the electric field of the unknown pulse, and the gate pulse multiply. The gate function can be any known function or it can also be a replica of the pulse itself.

The spectrograph function $S(\omega, \tau)$ contains all the information necessary to reconstruct the electric field of the pulse ${ }^{4}$. A pictorial representation of a spectrograph

\footnotetext{
${ }^{3}$ An Attosecond SPIDER method has also been proposed [88] but currently not implemented.

${ }^{4}$ Excluding CEP and GD terms.
} 
function is a music score. Figure 3.7, shows a music score for two pulses. Both pulses contain the same frequency content, however one pulse is notably shorter. The spectrograph function is often used in representing EUV pulse measurements.

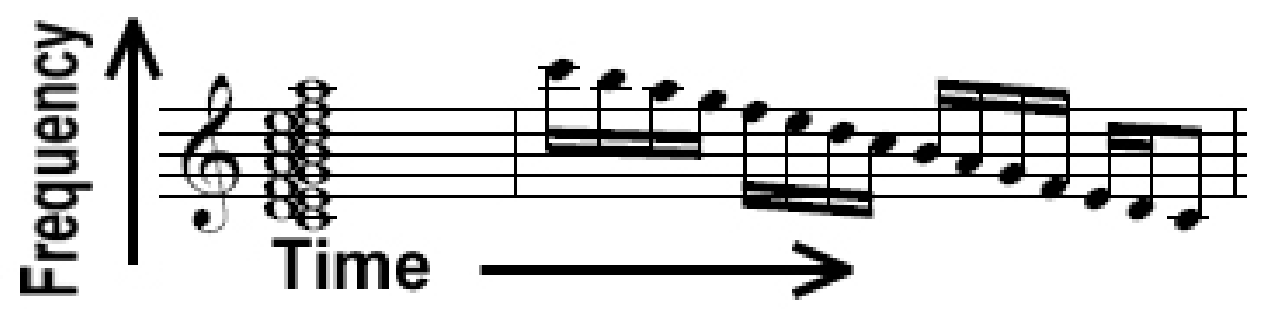

Figure 3.7: This is a music score that graphically represents a spectrograph function. The $\mathrm{x}$-axis is time and the $\mathrm{y}$-axis is frequency. The notes represent the intensity of the signals. There are two pulses shown in this spectrograph. The pulse on the left is a pulse with no chirp while the pulse on the right is negatively chirped .

Another method used for measuring picosecond x-ray pulses is a streak camera[89][90]. The concept is to convert the photons to photoelectrons in a drift tube. A voltage ramp is used to laterally deflect passing electrons in the drift tube, converting an axial position to a time dependent vertical position. The exact moment the photon produces the photoelectron dictates how long and what fields the electron will encounter. This then determines the arrival location on the streak camera's vertical detector plane. Knowing the applied voltages vs time allows the temporal reconstruction of the x-ray pulse. However, due to the limitations on the ramp of the applied electrostatic field, this technique is limited to a time resolution of $\sim 1$ picosecond. This is much larger than the duration of attosecond EUV pulses, therefore alternative techniques need to be implemented for attosecond EUV pulse measurements. 


\subsubsection{Pulse Measurements in the EUV}

Most techniques in EUV optics derive from the analogies, concepts, and techniques from visible light optics. Measuring attosecond pulses is no exception. However, there are many differences that make an all optical method in the EUV difficult. The reason is that there are no large nonlinear all optical effects in the EUV ${ }^{5}$. While the one photon absorption cross section in the EUV scales as $\sim(\hbar \omega)^{-3}$ the two photon cross section scales approximately as $\sigma_{2} \approx \sigma_{1}^{2}$ or $\sim(\hbar \omega)^{-6}[92]^{6}$. Due to the unfavorable scaling so far all-optical nonlinear effects were only used in pulse measurements with EUV pulses $\hbar \omega<30 \mathrm{eV}[91]$.

The nonlinearity requirement for pulse measurements is to allow for a multiplication of the electric fields of the pulse and a gate in the FROG measurement. In the previous section converting x-rays to electrons allowed for the direct interaction with an electric field. Suppose an EUV pulse enters a low density gas. Atoms in the gas will be ionized. The photoelectron will have a kinetic energy, $K E=\hbar \omega-I p$, where $\mathrm{KE}$ is the kinetic energy, Ip is the ionization potential of the atom, and $\hbar \omega$ is the energy of the photon. For a given (constant) gas pressure the number of photoelectrons produced is proportional to the number of EUV photons in the pulse ${ }^{7}$. The photoelectron wavepackets will also inherit the phase of the EUV pulse when

\footnotetext{
${ }^{5}$ There are nonlinear effects in the EUV that prodoce photoelectrons for example: two photo above threshold ionization[91].

${ }^{6}$ This justifies the statement of why CEP is not important to EUV attosecond pulses. As CEP is only important for nonlinear effects.

${ }^{7}$ This assumes the absorption cross section is constant for the energy range of the pulse.
} 
the electrons are from a well-defined transition, as in the case of ionization of noble gasses. This creates a direct mapping between the EUV optical pulse and the photoelectron wavepacket. The EUV intensity can be related to the number of produced photoelectrons and the kinetic energy is related to the frequency (photon energy).

Time resolution can be obtained by overlapping the EUV pulse with a high intensity IR pulse delayed by a time $\tau$. The photoelectrons will then experience a force proportional to the electric field of the IR pulse. This will in-turn modulate the kinetic energies and phases of the photoelectrons. Detecting the number of electrons as a function of kinetic energy and delay time $\tau$ produces an observable signal resembling a spectrograph. If the IR gating pulse is fully known, then the photoelectron bunch and hence the EUV attosecond pulse can be reconstructed. This method is the basis of the "attosecond streak camera"[93] and of the "frequency resolved optical gating for complete reconstruction of attosecond bursts" (FROG-CRAB) [94].

While the attosecond streak camera can only be used on isolated attosecond pulses the FROG-CRAB method can be used on both isolated pulses and pulse trains. For EUV pulse trains, another method, called RABBITT, that overlaps an IR pulse with the EUV pulse train in a noble gas is also used. Instead of observing the streaked electron, the signal is derived from a two-photon above threshold ionization process, where one of the photons is an EUV harmonic and the other is an IR photon. This method is called the "reconstruction of attosecond harmonic beating by interference of two-photon transitions" (RABBITT)[95][96][97]. 


\subsubsection{Attosecond Pulse Measurements}

To date, the shortest isolated pulses, measured at $\hbar \omega=80 \mathrm{eV}$, have had a duration of $80 \pm 5$ attoseconds[3]. This section describes the author's work on attosecond EUV pulse measurements at the Max-Planck Institute for Quantum Optics (MPQ) and Ludwig-Maximilians University (LMU) in Garching, Germany. The schematic for the measurement is shown in figure 3.8. A 3.5 femtosecond CEP cosine stabilized, 800 $\mathrm{nm}$, IR laser pulse was focused into a neon gas to produce high harmonic radiation. The EUV and the IR radiation co-propagated into the measurement chamber. In the measurement chamber, the EUV passed through a $300 \mathrm{~nm}$ zirconium (Zr) filter, used to filter the EUV pulses and remove the lower harmonics, producing an isolated pulse, while an adjustable aperture was used to adjust the IR intensity. The Zr filter blocks the discrete harmonic region and allows the transmission of the cutoff continuum. The GDD added by the filter compensates for the intrinsic chirp in the EUV pulse. The EUV and IR were then reflected off a two component dichroic mirror (for a detailed image of such an optic see figure 4.9 in chapter 4) and then focused into a FROG-CRAB detection system ${ }^{8}$. The inner component of the mirror was coated with a multilayer to reflect and focus the EUV (centered at $\hbar \omega=80 \mathrm{eV}$ ), while the IR was focused by the outer mirror. The two component optic is designed with the EUV optic inside the IR optic because the divergence angle of the IR is larger than the divergence

\footnotetext{
${ }^{8}$ The FROG-CRAB consisted of a neon gas target and a time of flight electron spectrometer.
} 
angle of the EUV ${ }^{9}$. To center the EUV/IR overlap in time, the EUV component of the optic could be moved relative to the outer IR component. Nanometer accuracy position in the two surfaces permitted attosecond resolution (e.g. $3 \mathrm{~nm}$ displacement resolution in the mirror translates to a 20 attosecond delay time accuracy between EUV and IR).

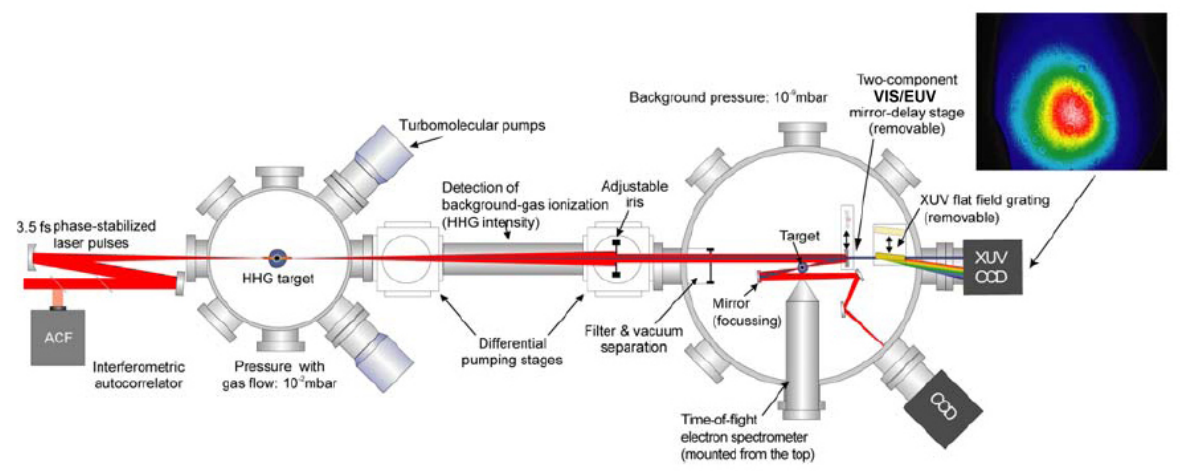

Figure 3.8: This figure shows the scientific setup of the HHG generation and pulse measurement chamber described in the text. Figure from collaboration with $\mathrm{MPQ} / \mathrm{LMU}[3]$.

The optics used for the EUV system are shown in figure 3.9. The optics consist of a $300 \mathrm{~nm}$ Zr filter and a Mo/Si multilayer mirror. The multilayer required a FWHM bandwidth of $\sim 28-30 \mathrm{eV}$ (see figure 1.5), higher than the band-width limit $(23 \mathrm{eV}$ ) due to a non-Gaussian pulse shap. A Mo/Si multilayer with $N=2$ bi-layer periods and $\gamma=.48$ was chosen, fabricated using ion beam deposition, and measured at the ALS beamline 6.3.2 (see section 1.2.4). A periodic multilayer was acceptable as the Zr filter nearly compensated for the chirp in the EUV under the given intensity

\footnotetext{
${ }^{9}$ As stated in the introduction (section 1.3.3) the HHG signal obtains its coherent properties directly from the driving laser[35].
} 
conditions.
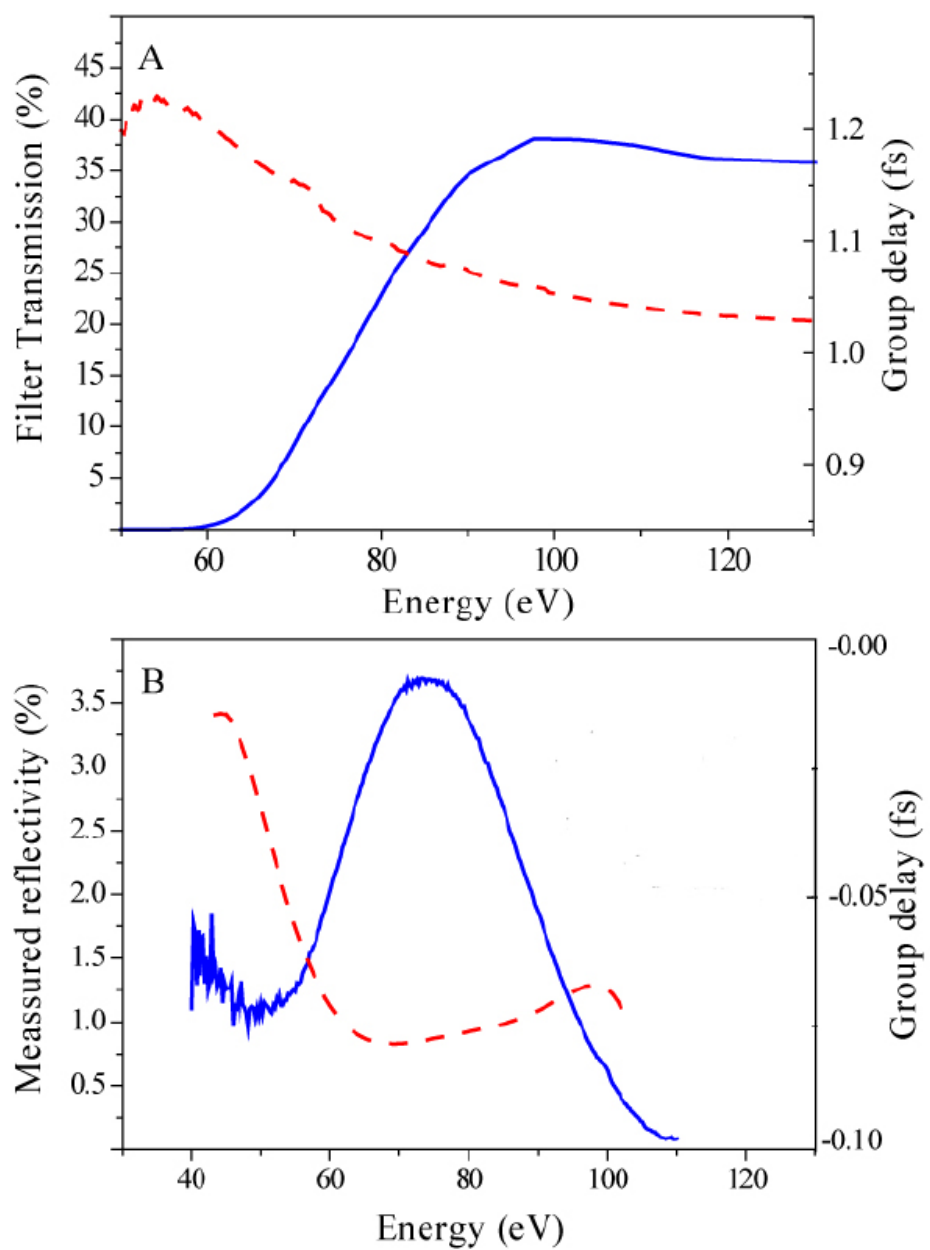

Figure 3.9: This figure shows the EUV optics used in the experiment. The top figure (A) shows the calculated transmission (blue curve) of a $300 \mathrm{~nm} \mathrm{Zr} \mathrm{filter[16].} \mathrm{The}$ red dashed curve is the GD for the filter, the GDD can be calculated by taking the derivative with respect to photon energy. The bottom figure (B) is the measured reflectivity of the Mo/Si multilayer (blue curve) and the group delay based on phase calculations. The multilayer reflectivity was measured at the ALS beamline 6.3.2. Figure from collaboration with MPQ/LMU[3].

The pulse measurement FROG-CRAB spectrograph is shown in figure 3.10A. The X-axis shows delay time between the EUV and the 3.5 femtosecond IR laser mirrors. The y-axis is the energy of the recorded photoelectrons. The color represents the 
relative number of the photoelectrons. The measurement used 126 separate photoelectron spectra with a delay of 80 attosecond between spectra and used $\sim 42,000$ EUV pulses for each photoelectron spectra. Based on the IR driving laser pulse measurement, used as a gate, a FROG reconstruction of the isolated EUV pulse is shown in figure 3.10B the FROG-CRAB reconstruction indicates a pulse duration of $80 \pm 5$ attoseconds FWHM. A GDD of $\phi^{\prime \prime}(\omega)=(1.5 \pm .2) \times 10^{-3} \mathrm{fs}^{2}$ was also determined.

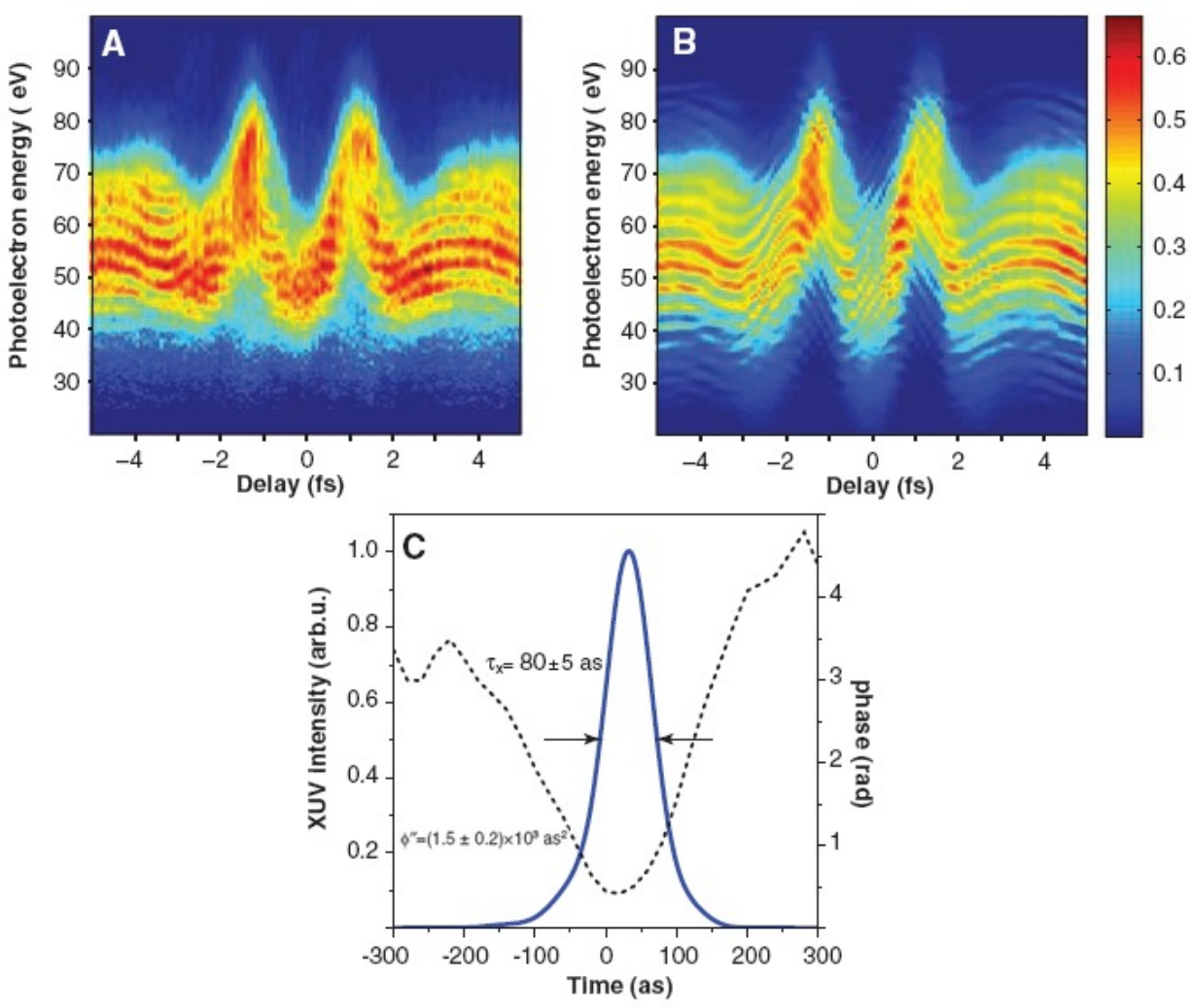

Figure 3.10: Subfigure A is the FROG-CRAB spectrograph taken from the pulse measurements and described in the text. Subfigure B is a FROG reconstruction of the attosecond pulse with the IR gate. Subfigure $\mathrm{C}$ gives the reconstructed intensity and phase for the 80 attosecond pulse. The GDD is calculated from the Fourier transform of the pulse. Figure from collaboration with MPQ/LMU[3]. 


\section{Chapter 4}

\section{Applications to Femtosecond Spin}

\section{Dynamics}

\subsection{Introduction to Femtosecond Spin Dynamics}

An area, where femtosecond process are currently being investigated, is ultrafast spin dynamics. In 1996, Beaurepaire and colleagues in a ground breaking demonstration showed that the demagnetization of a ferromagnet $3 d$ transmission metal (Nickel) took less then 200 fs[98]. This surprising result from Beaurepaire used visible light magneto-optical Kerr effect (MOKE)[99] measurements to measure the magnetization of the sample as a function of time delay after a femtosecond heating pulse (see figure 4.1). The result was surprising as the demagnetization was expected to take $100 \mathrm{~s}$ of picoseconds. The interpretation that this effect was magnetic in origin remained 
contested and controversial for a long time[100]. Only recently, with the adoption of SXR techniques, has it been shown that the observed demagnetization is a real magnetic effect[101]. This pioneering result by Stamm et. al.[101] also demonstrated the practical limits of using current synchrotron sources for femtosecond spin studies. The experiment demonstrated the femtosecond nature of the magnetic effect, however, the underlining physical process remains unknown.

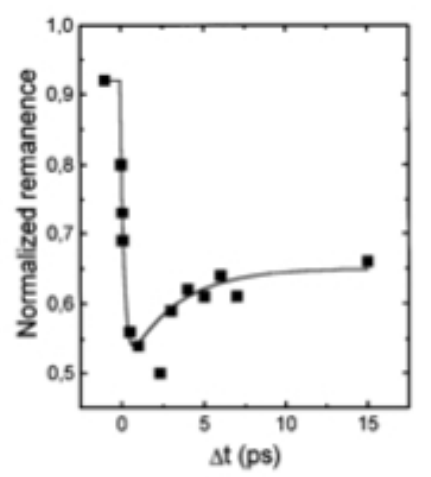

Figure 4.1: This is the result of Beaurepaire's MOKE experiments to determine the demagnetization of nickel. The experiment shows normalized Kerr rotation at remanence (no magnetic field applied) with respect to delay after a $60 \mathrm{fs}, 7 \mathrm{~mJ} / \mathrm{cm}^{2}$ heating pulse. The demagnetization of the sample is less than under 200 femtoseconds. Figure from [98].

This section gives a brief overview on the current state of femtosecond spin dynamics. It also reviews recent experiments and attempts to explain the physical mechanisms for the fast demagnetization. 


\subsubsection{Models of Femtosecond Magnetics}

The current understanding of femtosecond spin dynamics and demagnetization is controversial, due to the complexity of energy and momentum transfer in a ferromagnetic system on the feec time scale. The thermodynamics of magnetic systems can be described using a three temperature model [98] as illustrated in figure 4.2. For a nonmagnetic metal, the system can be thought of as containing only two components: the electron gas in the conduction band and the ionic cores in a lattice. Using the Born-Oppenheimer approximation ${ }^{1}$, the motion (and hence temperature) of the electrons is independent of the lattice. The ion cores can be excited via mechanical motion such as pressure and deformation of the material (i.e. phonons), while the electrons can be excited by currents or optical pulses ${ }^{2}$. For a magnetic material a third component is needed to describe the spin system (i.e. another degree of freedom).

In a magnetic system, a third temperature is given to describe the degree of freedom for spins in the material. This energy reservoir includes exchange interaction and spin-orbit interactions. The magnetic temperature (energy term) can also be described by magnetic oscillations (i.e. spin waves), also referred to as magnons. A magnon is the equivalent excitation of thermal energy in a magnet, as a phonon excitation is to the thermal energy in the lattice.

What occurs when an intense femtosecond laser pulse strikes a magnetic systems

\footnotetext{
${ }^{1}$ This approximation states that the electron motion can be separated from the nuclear motion.

${ }^{2}$ Electromagnetic radiation does transfer energy to the atom cores; however, as the mass of the ion cores is much larger than the mass the electrons the energy transfer is negligible.
} 


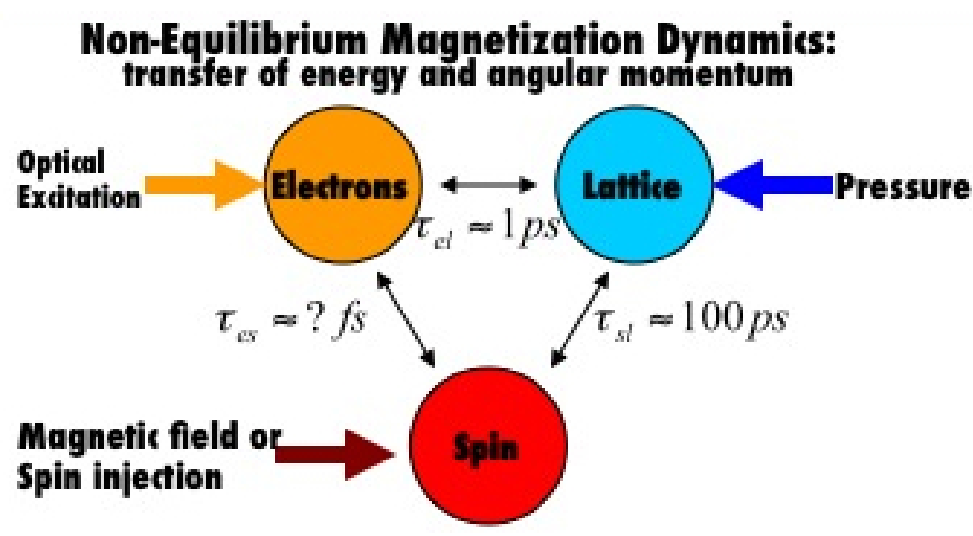

Figure 4.2: This figure shows the three temperature model of a magnetic system and the various channels for the transfer energy and momentum. Each energy reservoir can be at a different temperature. In equilibrium they are at the same temperature. The question mark for electron-spin interactions, $\tau_{e s}$, is the unknown femtosecond transfer of orbital momentum being studied[102].

is a multistep process. First, the electrons are excited when the energy of the pulse is absorbed which creates non-thermal electrons. These electrons, then, thermalize quickly (less than $500 \mathrm{fs}$ ) to an electron temperature, $T_{e}$. During and after the thermalization/relaxation process the electrons scatter off the lattice and cause phonons. This transfers energy and reduces the electron temperature, $T_{e}$, while increasing the lattice temperature, $T_{l}$. All of this occurs on a time scale of the order of one picosecond (1000 fs). The energy is then transfered to the spin system via phonon-magnon interactions $^{3}$ on a slow, 100s of picosecond, timescale. In nonmetals this theoretical explanation accurately describes the experimental demagnetization. For example the time resolved demagnetization of nonmetal $\mathrm{FeBO}_{3}$ with antiferromagnetic ordering is around 700 picosecond[103]. However, this is not the case in metals, as demonstrated

\footnotetext{
${ }^{3}$ This interaction can be thought of as spin-orbit coupling of the magnetic ions through magnetostriction[103][104].
} 
by Beaurepaire and confirmed by others[98][105][106] using various methods such as photoemission from two-photon absorption. The demagnetization process occurs on a timescale of a $100 \mathrm{fs}$ but the exact mechanism is still unknown[107].

To understand the mechanism, other election-spin interactions have been researched including relativistic electron bunch excitation[108], and inverse-Faraday effect[109][110]. The problem with a direct electron-spin interaction, i.e. without involving the slow lattice interactions, (in figure 4.2) is that it would violate the conservation of angular momentum. In other words if two electrons collide and scatter, the electrons cannot change their spin(e.g. number of spins up to down must be conserved).

One of the possible mechanisms that is discussed to explain femtosecond demagnetization is the use of Elliot-Yafet scattering[111][112]. This method involves a conduction electron spin-flip scattering off an impurity atom or scattering off a phonon in the lattice. This allows the electron to spin flip with the angular momentum of the electron transfered to the lattice. This model relates the Gilbert damping term[113], $\alpha$, of the Landau-Lifshitz-Gilbert magnetic dynamic equation ${ }^{4}$, and the Curie temperature, $T_{c}$, to the demagnetization time scale: $\tau_{m}=c_{0} \hbar / k_{B} T_{c} \alpha$. However, recent experimental measurements have indicated that additional coupling methods occur that are neglected in this model[107]. Other theories include the Stoner type excitations[114] or significant changes in the band structure of the metal caused by

\footnotetext{
${ }^{4}$ The Landau-Lifshitz-Gilbert equation is used to describe the precessional motion of the magnetization in a solid.
} 
the heating pulse[101].

Currently the only measurement to confirm the magnetic effect uses time resolved X-ray magnetic circular dichroism (XMCD) from a slicing undulator[115][101]. This experiment utilized a femtosecond IR laser pulse to heat (pump) the sample and a sliced (femtosecond) undulator SXR probe pulse. The magnetization was determined by observing transitions from $2 p_{1 / 2}$ and $2 p_{3 / 2}$ to the conduction band using circularly polarized SXR radiation. This technique, along with EUV magnetic techniques will be discussed in the following section.

\subsection{Comparison of Magnetic Probing Techniques}

EUV and SXR magnetic probing techniques are powerful tools that allow for the observation of element specific magnetic effects. In this section, a comparison is given for magneto-optic techniques in the EUV to those in SXR. This is followed by a description of possible femtosecond sources to be used in femtosecond spin dynamic experiments.

\subsubsection{Magnetic Effects in the EUV/SXR}

Magneto-optic effects for visible wavelengths are well known[116][117]. In magnetooptic measurements linearly polarized light is reflected off or transmitted through a magnetic sample. The axis of linear polarization will rotate and the magnetic field 
determines the rotation angle. If the sample is measured in reflection, it is called the magneto optical Kerr effect (MOKE). If it is measured in transmission it is called a Faraday rotation. Quantum mechanically, magneto-optic effects are described by Zeeman splitting. Light with right circular polarization will interact differently with a magnetic material than light with left circular polarization. This is due to the selection/transition rules. Circularly polarized light requires that $\Delta m$ in a dipole transition be +1 for right circular polarization, and -1 for left circular polarization. The difference of absorption for right and left circular polarization also leads to a difference in the real part of the index of refraction by the Kramers-Kronig relationship. As linearly polarized light can be transformed into a basis consisting of right and left circular polarization, this lead to rotation of linearly polarized light.

Analogous effects occur in the EUV and SXR range of the spectrum. However, these effects only occur at absorption edges. Measurement techniques include X-ray magnetic circular dichroism (XMCD)[118][119][120][121], Faraday rotation[122][123] and MOKE[124]. EUV and SXR techniques probe electron transitions between occupied to unoccupied states. As the initial electron state is known and occupied, absorption is a way to measure the density (number) of unoccupied states. For $3 d$ magnetic materials, the magnetization occurs because of the difference in populations of the spin up electrons to the spin down electrons in the material. Figure 4.3 shows a simplified band model for iron separated into spin up and spin down electrons. For dipole transitions of circularly polarized light, the electron transition must match the 


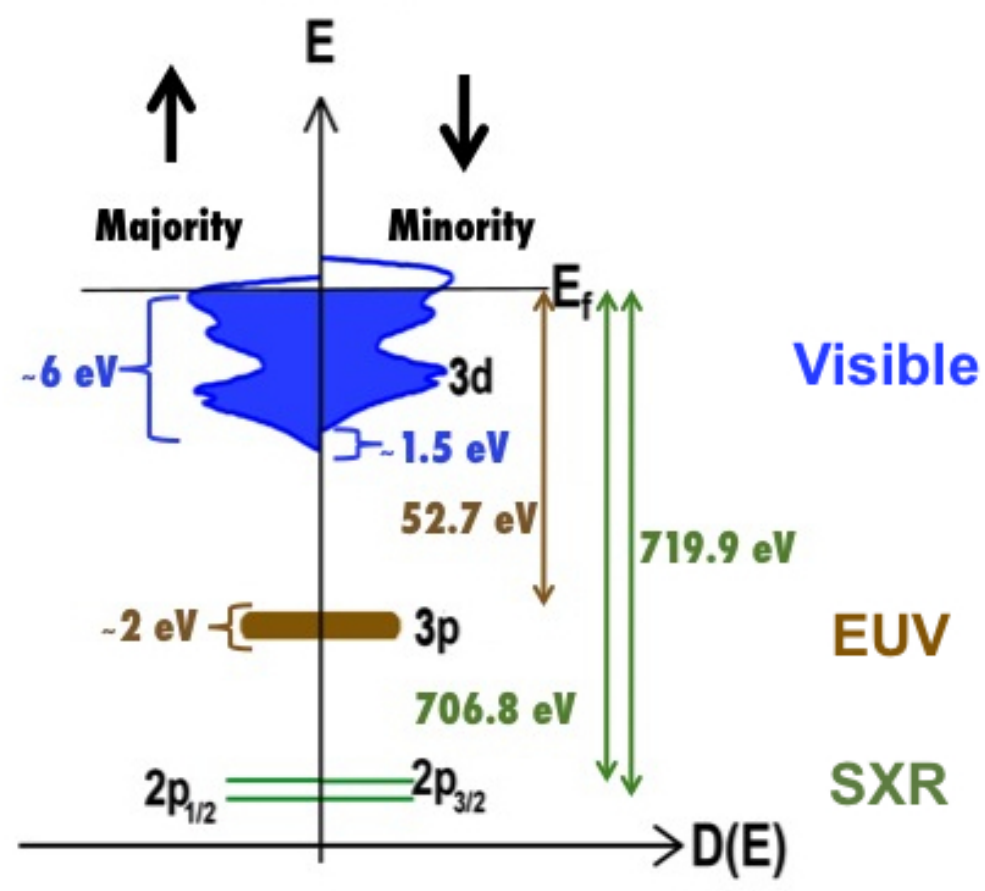

Figure 4.3: This figure shows a simplified band picture of iron. The selection rules require that a right or left circular polarized photon only interact with either the majority or the minority spin carrier. Notice also that the $2 \mathrm{p}$ band is spin orbit split while the $3 p$ band is exchanged split and broad.

selection criteria $\left(l= \pm 1 \mathrm{p} \rightarrow \mathrm{d}\right.$ transition ${ }^{5}, \Delta m=+1$ right circular, $\Delta m=-1$ left circular ). This means that a right circularly polarized photon will only interact with the majority carriers (spin up electrons) while a left circularly polarized photon will only interact with the minority carriers (spin down electrons) $)^{67}$. As all the $2 p$ and $3 p$ states are filled, the number of free states above the Fermi energy determine the amount of absorption. There is a critical difference between absorption in the $2 p$

\footnotetext{
${ }^{5}$ The $\mathrm{p} \rightarrow \mathrm{s}$ transitions are negligible.

${ }^{6}$ Transitions where the electron's spin is flipped are small or forbidden[125].

${ }^{7}$ If the magnetic field is reversed then the left circularly polarized light will interact with the majority and the right with the minority.
} 
(SXR) and the $3 p(\mathrm{EUV})$. The $2 p$ SXR levels are spin orbit split $\left(2 p_{1 / 2}\right.$ and $\left.2 p_{3 / 2}\right)$ and localized, while the $3 p$ EUV levels are broader and exchanged split[122] and itinerant (delocalized) states. Because the $2 p$ levels are spin orbit split, sum rules are used to determine how much of the magnetization is from spins and how much is from orbital angular momentum[126][125]. In the $3 p$ level absorption this is not the case, the electrons are spread out is a delocalized band with a bandwidth of a few $\mathrm{eV}$. Meaning the physics of the EUV states are not the same as the discrete SXR case or the same as continuous visible transition; they are somewhere in-between. Because the $2 p$ and $3 p$ states couple to different aspects of a magnetic system, they can provide complementary information about the dynamics of the system. One other aspect of note, is that the XMCD signal of the $3 p \mathrm{~L}_{2,3}(\sim 50 \%)$ is larger than the XMCD signal at the $2 p \mathrm{M}_{2,3}$ edge $(\sim 10 \%)$.

\subsubsection{Free Electron Lasers Vs. High Harmonic Generators}

There are many critical parameters in choosing a light source for dynamics experiments. The critical parameters are the photon energy, pulse duration, photons per pulse, and polarization. The photon energy determines the magnetic material as it needs to correspond to an absorption edge. The photon energy (wavelength), along with the optics, determines the achievable spatial resolution, which is critical for certain experiments. The pulse duration determines the temporal resolution and places a limit on the physical processes that can to be studied. As can be seen in figure 
4.4 the processes of interest for femtosecond spin dynamics are spin fluctuations and exchange interactions. Photon flux is critical as it determines whether a single pulse, short time duration experiment is feasible. If the flux is low, then the experiment will require numerous pulses. The increase in required repetitions is a critical concern as the process under study may not be perfectly repeatable, or repeatable at all, creating significant errors in the measurement. Polarization control is particularly important for the study of spin dynamics.

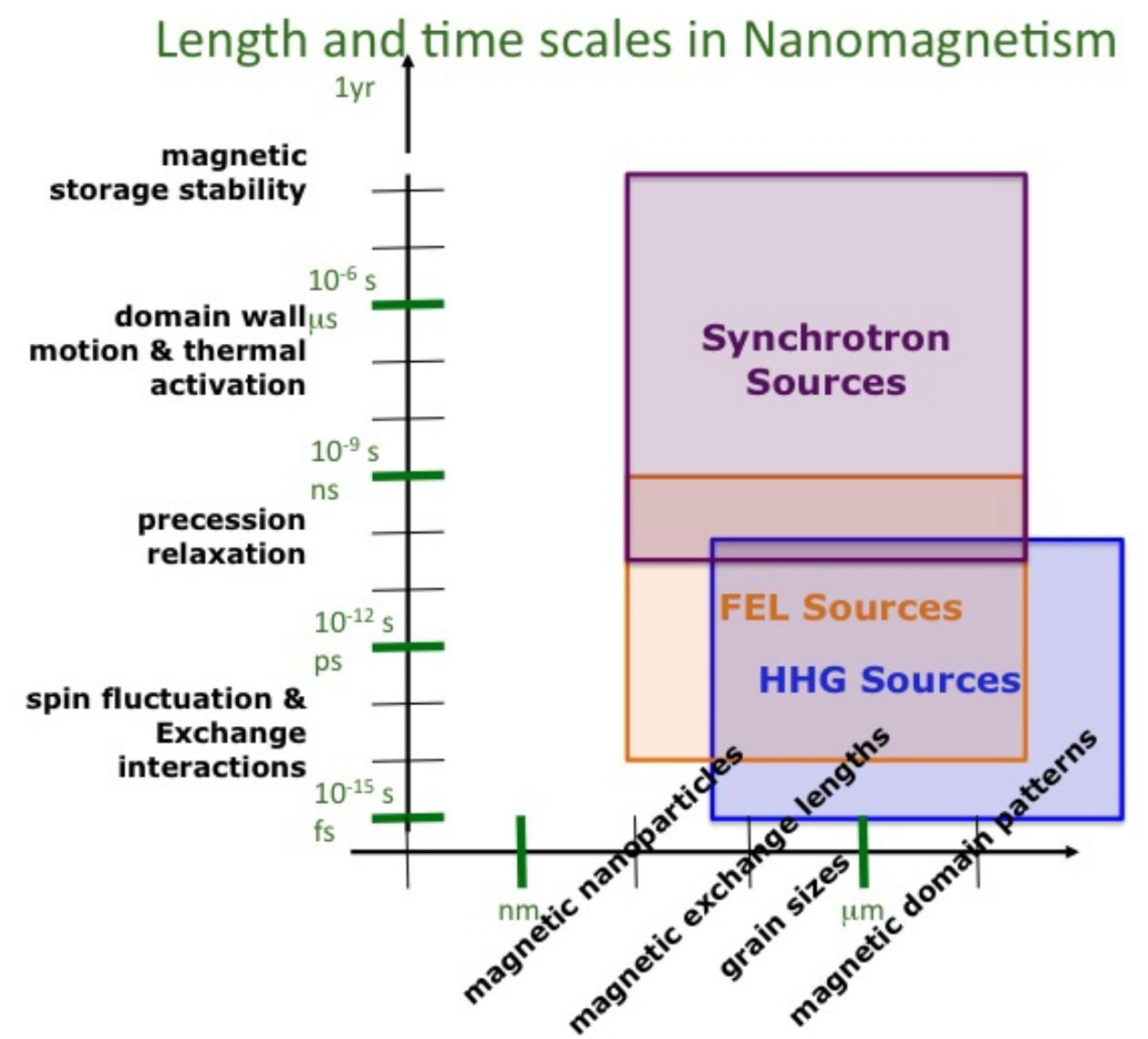

Figure 4.4: The graph is of the various magnetic time scales and spatial scales. Shown are also the magnetic probing ranges for EUV and SXR radiation with synchrotrons, FEL and HHG sources. 
The current generation of synchrotron sources are limited for the study of spin dynamics ${ }^{8}$. Synchrotron radiation nominally consists of 100 picosecond pulses[1]. One method used to obtain better time resolution at a synchrotron is to slice the electron bunch with a femtosecond laser[115], laterally displace a section of the bunch, and thus providing a shorter probe. This method has been used for the magnetic experiments by Stamm[101]. However, slicing suffers from low photon flux, typically 1000 photons $/(0.1 \%$ bandwidth $)$ pulse[115]. Additionally, timing jitter leads to significant temporal error bars of around 100 femtoseconds.

Alternatively it is just becoming feasable to study fs spin dynamics with high harmonic generator and free electron lasers. An advantage of using HHG sources is the ability to generate femtosecond EUV pulses, sometime extendable to the attosecond regime. However, HHG is limited in the number of photons per pulse generated in the spectral region of greatest interest for spin dynamic systems, the L-edges of Fe, Co and $\mathrm{Ni}$ at 700-800 eV. Alternatively HHG might be used at the M-edges of these magnetic materials, typically at photon energies of 50-70 eV, albeit with less sensitivity to spin dynamics. In an isolated harmonic HHG can produce only $6 \times 10^{8}$ photons/pulse in a single harmonic at $54 \mathrm{eV}$. Current technology has limited high harmonics with reasonable flux to photon energies of about $30-50 \mathrm{eV}$, with decreasing flux to 100 $\mathrm{eV}$ and above[128]. In other words, current HHG sources cannot reach the $2 \mathrm{p}_{1 / 2,3 / 2}$ transition $\left(\mathrm{L}_{2,3}\right.$ edges) in the $3 \mathrm{~d}$ transition metals with enough flux to permit single

\footnotetext{
${ }^{8}$ Certain magnetic dynamics, such as the domain wall motion, occurs on the order of picoseconds to nanoseconds and can be measured at standard synchrotrons[127].
} 
pulse femtosecond measurements.

Free electron lasers (FELs) provide an additional option, albeit just emerging, for the study of femtosecond magnetic dynamics[129]. Free electron laser radiation is similar to undulator radiation[1] but more intense, coherent, and most importantly high photon flux in a single pulse. The intensity of the radiation produced by an undulator is proportional to the number of electrons, $\mathrm{N}$, in the electron bunch. In a free electron laser, the electrons all radiate coherently ${ }^{9}$ with an intensity proportional to $\mathrm{N}^{2}$. FEL's are expected to generate as many as $10^{12}$ spatially coherent photons in a single EUV or SXR pulse of about 200 fs duration. The current state of the art FEL (FLASH) facility operates at a photon energy of $90 \mathrm{eV}$ with $6 \times 10^{12}$ photons/pulse and a duration of less than $50 \mathrm{fs}[130]$. This pulse energy is enough to destroy most samples $[131]^{10}$. At this time there is only one free electron laser operating in the EUV[130]. Within the next year or two SXR free electron lasers(such as LCLS at Stanford[132]) are expected to come on line and be able to operate at the $2 \mathrm{p}$ edges of $3 \mathrm{~d}$ magnets. This will provide unique opertunities to probe spin dynamics with appropriate photon energies and pulse durations. In the meanwhile we are pursuing the use of HHG pulses at the M-edges.

\footnotetext{
${ }^{9}$ The electrons become coherent because the produced radiation from the undulator acts on the electrons to form micro-bunches one wavelength apart. Each micro-bunch radiates in phase with all the other electrons in the bunch.

${ }^{10}$ One solution is to use many identical samples, one for each time delay.
} 


\subsection{Femtosecond Time Resolved Magnetic Mea- surements in the EUV}

This section describes the author's preliminary experiment on femtosecond time resolved EUV magnetic circular dichroism in collaboration with the Parmigiani group in Trieste, Italy[133]. The experiment utilized a ferromagnet permalloy $\left(\mathrm{Fe}_{20} \mathrm{Ni}_{80}\right)$ sample, and it is the first in a series of experiments to obtain element specific spin dynamic information. The ultimate goal is to develop a scientific instrument that can give elemental specific interactions in spin systems. For example, a ferrimagnet containing both iron and nickel would be interesting to study as the instrument would be able to observe the demagnetization of the iron atoms independently to that of the demagnetization of the nickel atoms. The following section will describe the experimental setup, optics development, and first results of static measurements on permalloy. These measurements will be compared to synchrotron measurements.

\subsubsection{Experimental Setup}

For this experiment, the author chose to use a high harmonic light source as FEL sources are not currently available. As described in section 4.2.2, the choice of using HHG currently limits the experiment to the $3 \mathrm{p} \rightarrow 3 \mathrm{~d}$ transition $\mathrm{M}_{2,3}$ edges of iron and nickel at 50-70 eV, as there is insufficient flux to make single pulse measurements involving the $2 p$ L-edges near $700-800 \mathrm{eV}$. Figure 4.5 shows the experimental setup. 
The HHG system used is the T-REX lab located at the Elettra Synchrotron in Trieste, Italy. The high harmonic radiation was produced with a $2 \mathrm{~W}, 800 \mathrm{~nm}$, Ti-sapphire laser working at $1 \mathrm{kHz}$ repetition rate. The pulse duration of the laser was approximately 50 fs. An intensity spectrum from the high harmonic source is shown in figure 1.11. The author chose the 35th harmonic corresponding to the $\mathrm{Fe} \mathrm{M}_{2,3}$ absorption edge at $54 \mathrm{eV}(22.9 \mathrm{~nm})$. The $\mathrm{Fe}_{2,3}$ absorption edge is located at $52.7 \mathrm{eV}$, however as stated is a few eV wide. Based on synchrotron measurements $54 \mathrm{eV}$ produced a large dichroic signal.

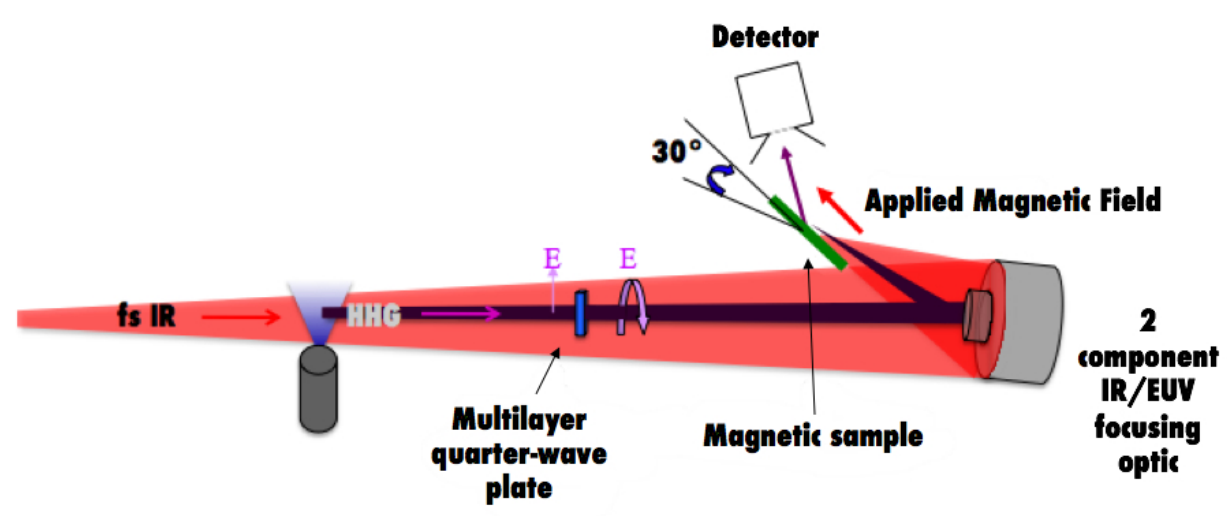

Figure 4.5: This is the experimental setup for the femtosecond time resolved EUV MCD The HHG produces EUV radiaton co-propagating with the driving IR field. The EUV is transmitted through a multilayer quarter wave plate, while the IR passes around the outside. The EUV and IR are then focused off a two component pump/probe mirror and onto the sample. My moving the EUV inner mirror in the pump probe setup with respect to the outer mirror, a femtosecond time delay can be obtained. The EUV reflects off the sample while the IR pump locally heats the channel electron multiplier detector.

Downstream the HHG cell the EUV and IR co-propogate to the multilayer quarter- 
wave plate (see sections 2.5 and 4.3.2). The multilayer quarter-wave plate circularly polarizes the 35th harmonic at $54 \mathrm{eV}$. The other harmonics pass through and the IR is reflected off the multilayer. A significant part of the IR passes around the edge of the multilayer quarter-wave plate and will be used as the probe. The light, then, impinges upon a two component mirror. The outer mirror is gold coated and reflects the IR while the inner mirror uses a multilayer to select and focus the EUV. The inner EUV mirror can be moved independently with respect to the IR outer mirror along the optical axis. This allows for a variable femtosecond time-delay to be obtained. The light is then focused on the sample where the IR acts as a pump to demagnetize the sample and the EUV probes the magnetic dynamics. Also, the sample stage has a magnetic field that can be applied in the plane of the sample. The reflected EUV then enters a channel electron multiplier that measures intensity of the signal. A channel electron multiplier was chosen as a detector as it is insensitive to the IR signal. The signal is then used as input in either a digital picoamp meter or a lock-in amplifier that is locked to the laser frequency. Based on the multilayer bandwidth, the pulse duration of the isolated harmonic was slightly smaller than that of the IR pump. This gave a time resolution of the order the IR duration of $50 \mathrm{fs}$.

\subsubsection{Optic Development}

As the $\mathrm{M}_{2,3}$ absorption edges of iron and nickel are 52.7 and $66.2 \mathrm{eV}$ respectively[83] the author chose to form a multilayer mirror of zirconium/aluminum ( $\mathrm{Zr} / \mathrm{Al})$. The 
material combination was chosen because the Al edge at $72.5 \mathrm{eV}[134]$ makes it relatively transparent and thus gives the multilayers better performance. Zirconium was chosen because of its known interface compatibility with $\mathrm{Al}[135]$. Four multilayer optics were produced, two focusing and two quarter-wave plates, one set for the iron edge and one set for the nickel edge.

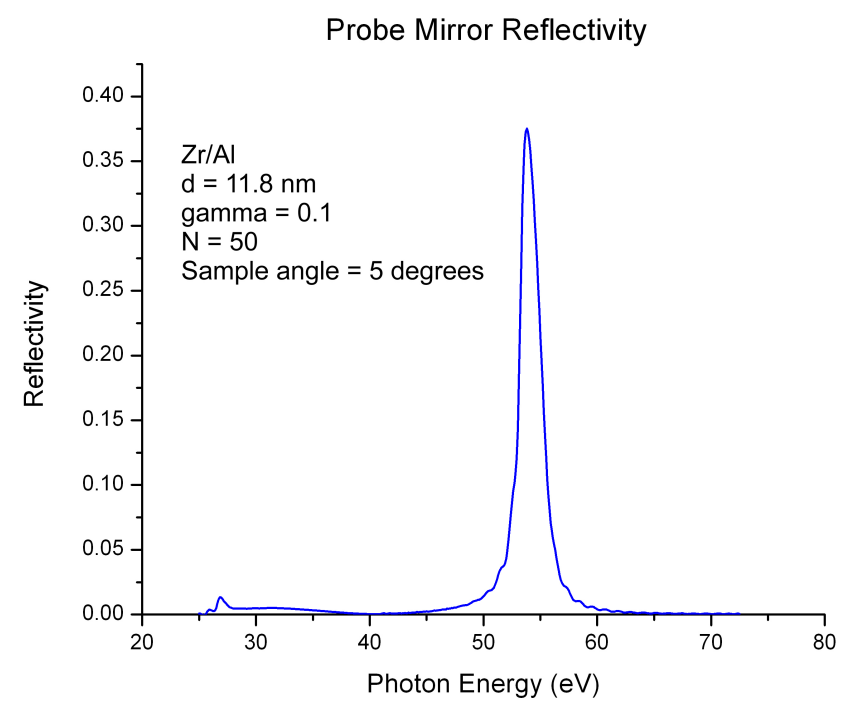

Figure 4.6: This is the measured reflectivity curve for the inner pump mirror designed for the $\mathrm{Fe}_{2,3}$ edge $52.7 \mathrm{eV}$. This mirror was designed to have high reflectivity peak at $54 \mathrm{eV}$ corresponding to the peak of the magnetic dichroic signal a bandwidth of $4.5 \mathrm{eV}$. Giving a large rejection (factor of 20) of neighboring harmonics.

The multilayer focusing optic was designed with two parameters in mind, high reflectivity and suppression of neighboring harmonics. The author decided to produce multilayers with d-spacing $=11.8 \mathrm{~nm}, \gamma=0.1(\mathrm{Zr} / \mathrm{d})$ and $N=50$ bilayers. This saturated the reflectivity and gave a factor of 20 suppression of the nearest neighbor harmonics (3 eV away). Figure 4.6 shows the measured reflectivity curve for the 
multilayer optic at the operating angle of 5 degrees from normal.

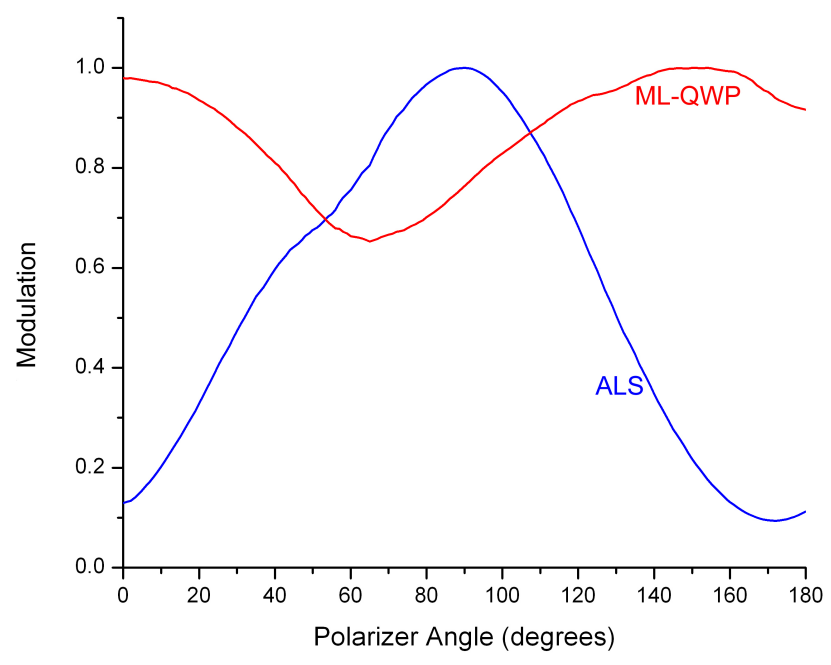

Figure 4.7: This figure shows the degree of polarization of the multilayer quarter wave plate designed for $54 \mathrm{eV}$. It was measured at the ALS beamline 6.3.2.

While developing the quarter-wave plate, it became necessary to explore the interfaces of $\mathrm{Zr} / \mathrm{Al}$ to accurately model and predict the properties of the quarter-wave plate. The interfaces of $\mathrm{Zr} / \mathrm{Al}$ multilayer have been relatively unexplored. Based on reflectivity measurements at different gamma values and interface barrier testing (using boron-carbide $\mathrm{B}_{4} \mathrm{C}$ at alternating interfaces), it was estimated that the $\mathrm{Al}$ on top of Zr interface was sharp (no measurable interdiffusion) and the $\mathrm{Zr}$ on top of $\mathrm{Al}$ had an interdiffusion of $\sim 1 \mathrm{~nm}$. Due to the incomplete knowledge of interface composition and roughness, compared to the more well known Mo/Si, the performance of multilayer quarter-wave plates was expected to suffer slightly. For the top layer material, it was determined that the optical properties of aluminum oxide produced lower reflec- 


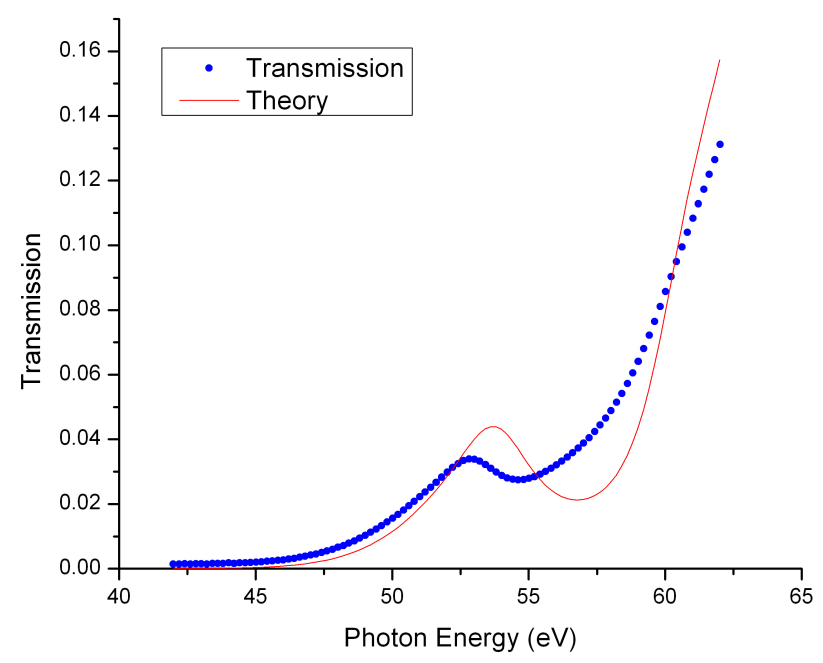

Figure 4.8: The transmission of the multilayer quarter wave plate at the $45^{\circ}$ angle (Brewster angle) with linearly polarized light. The azimuthal angle is $50^{\circ}$ for linearly polarized light. This is the angle chosen for polarization measurement in figure 4.7.

tivity than those of zirconium oxide. For the multilayer quarter-wave plate, both the top and bottom layers are Zr. Measurements of the multilayer quarter wave plates were performed in the same manner as described in section 2.5. The polarization curve and transmission measurements are shown in figures 4.7 and 4.8.

\subsubsection{Preliminary Results}

The optical setup of the experiment in the measurement chamber described in figure 4.5 is shown in figure 4.9. The author and colleagues chose to start with static magnetic contrast measurements, as no published work has been done using circularly polarized HHG radiation. These static measurements would then be compared to 


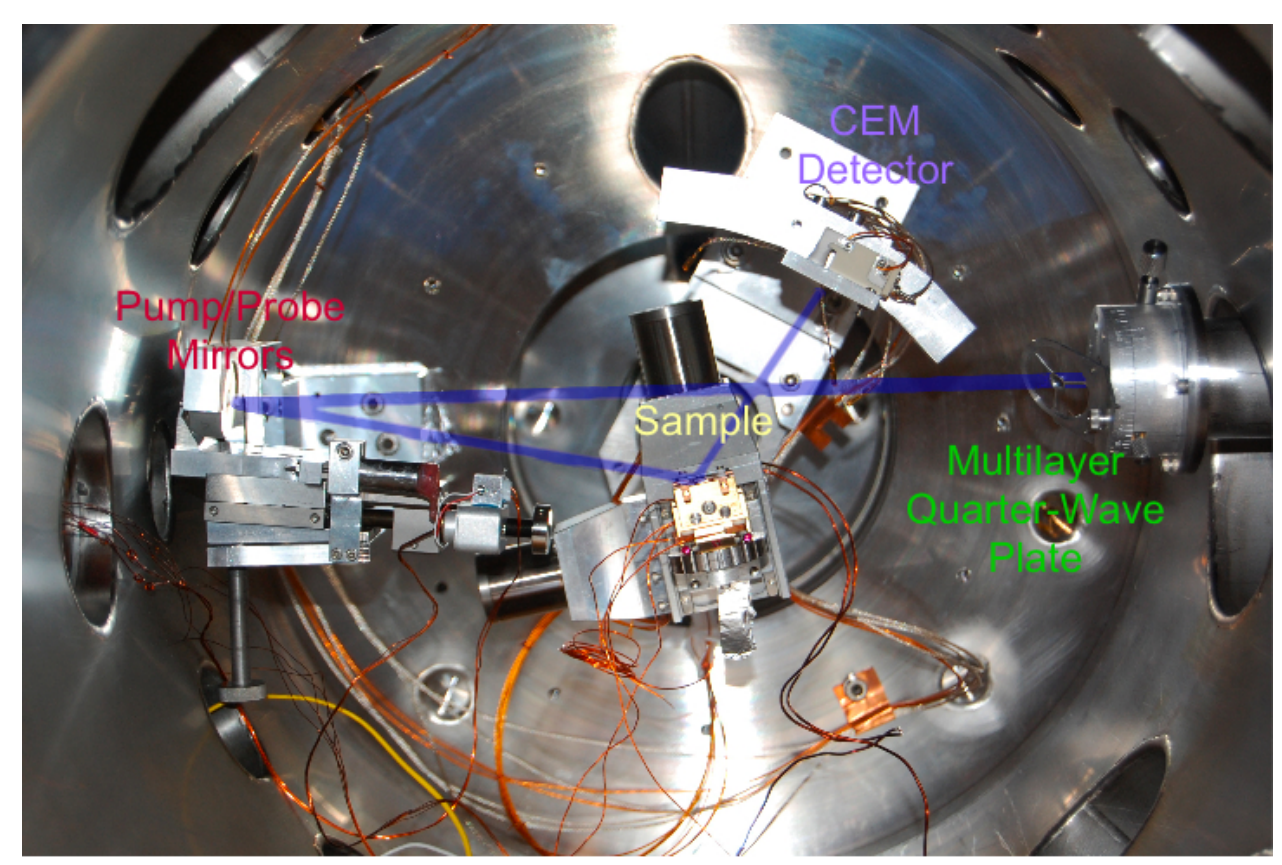

Figure 4.9: The HHG experimental setup described in figure 4.5. The optical path for the EUV is in light blue.

static measurements taken at the ALS. During the preliminary static measurements, an Al filter is placed between the measurement chamber and the HHG chamber. This is to block the IR light and to act as a pressure isolator between the chambers. The experimental results of the static measurement are shown in figure 4.10. During measurements with the HHG source positive and negative magnetic fields were alternated at varying field strength. The coercivity results agree with the measurements taken at the ALS beamline 6.3.2 (figures 4.12 and 4.13) of 1.8 Oersteds, as does the ratio of dichroic signal. Figure 4.11 is a derived hysteresis loop based on the measurements of figure 4.10. To the author's knowledge, these are the first results of the production of circularly polarized high harmonic light. 


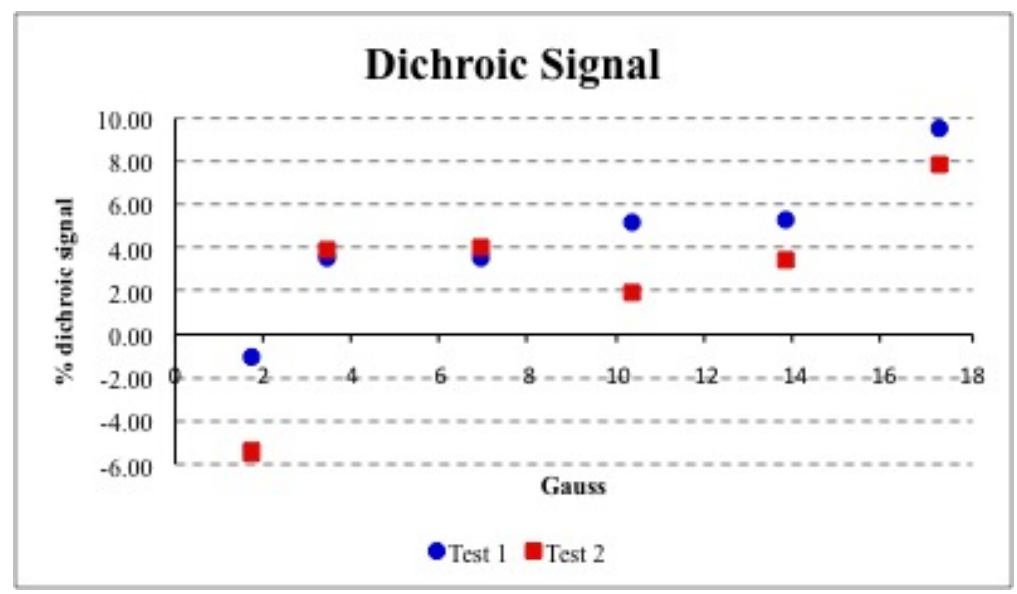

Figure 4.10: The experimental results of a static HHG measurement with a permalloy sample at the iron $\mathrm{M}_{2,3}(54 \mathrm{eV})$ edge. The measurements were performed at various field strengths in an alternating fashion of positive and negative fields. The blue dots represent the dichroic signal when the field increases in strength, while the red points indicate the field while the magnet decreases in strength. Below 1.8 Oe there is no way of controlling the direction of the magnetic field.

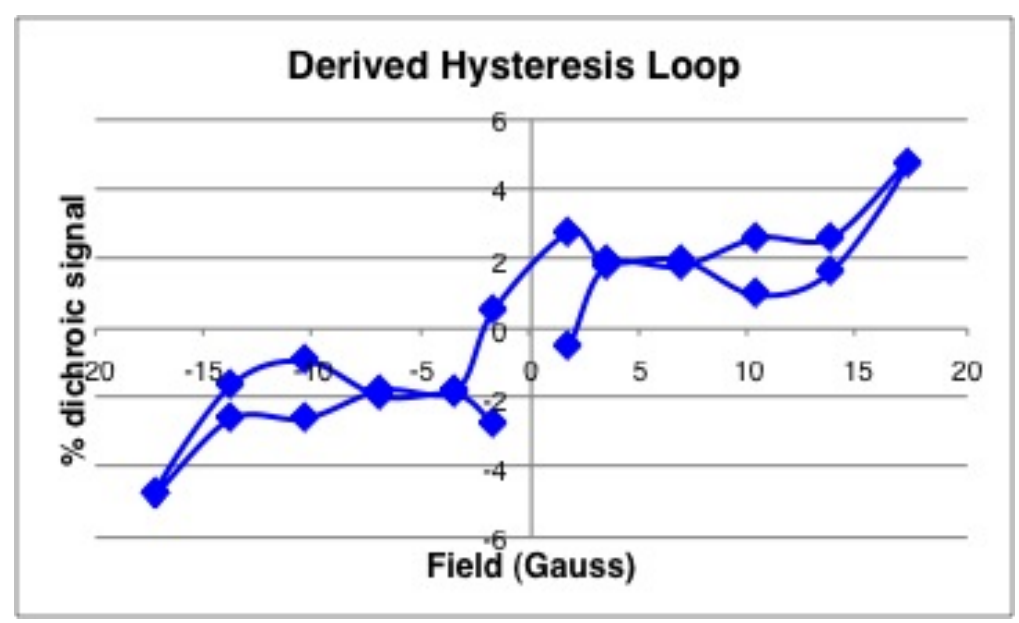

Figure 4.11: The derived hysteresis loop from the static HHG measurements given in figure 4.10 . 


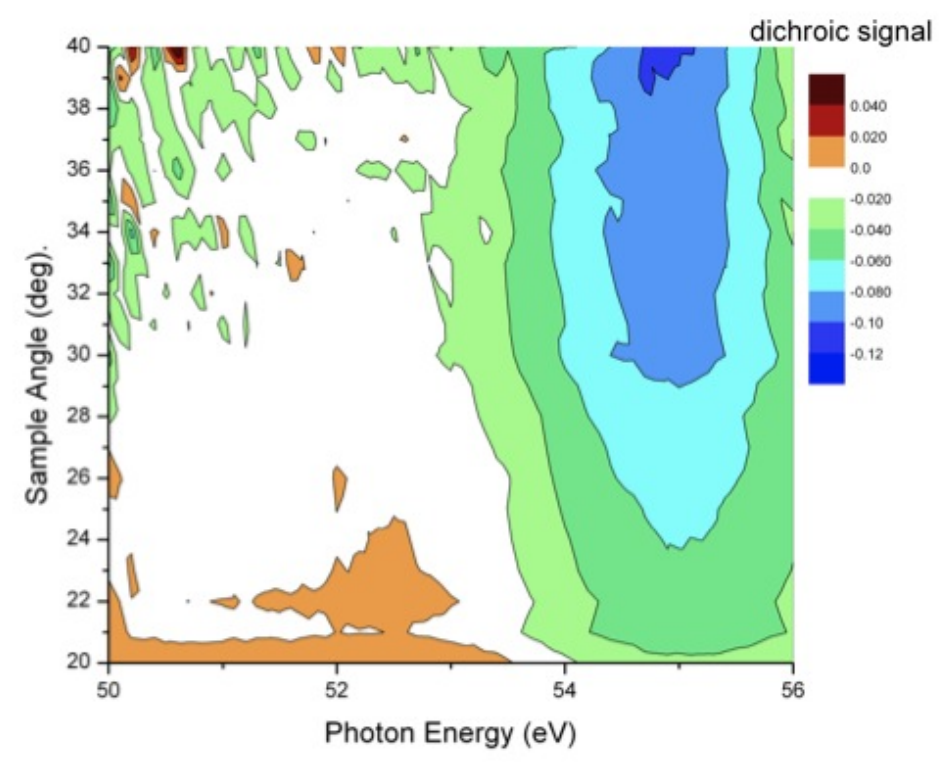

Figure 4.12: This figure is a plot of the strength of the dichroic signal as a function of photon energy and angle of incidence (degrees from grazing) as measured at the ALS. This data helped assess what energies and angles to operate the HHG experiment at. The peak dichroic signal was $10 \%$ and measurements agreed with [121].

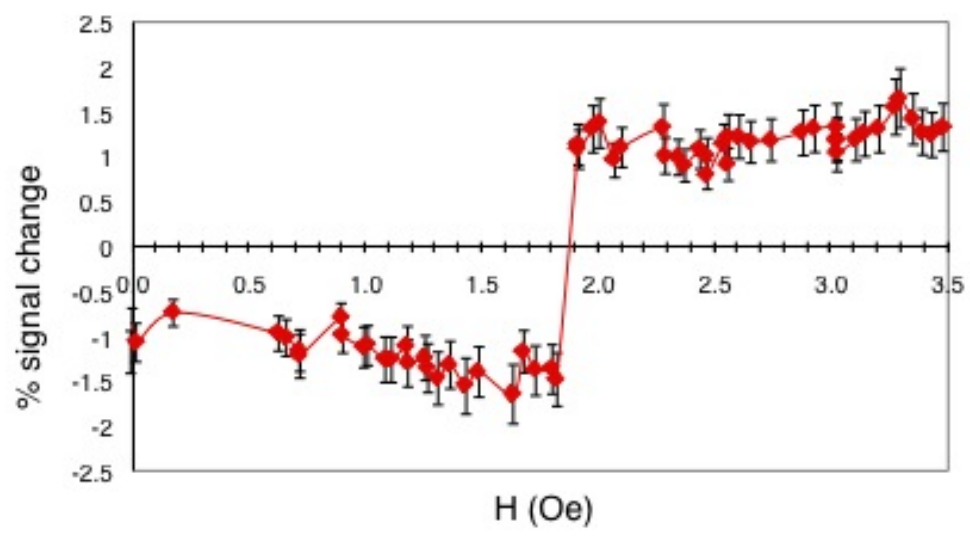

Figure 4.13: This figure is part of a hysteresis loop measurement of permalloy completed at ALS beamline 6.3.2 using a multilayer quarter wave plate at the iron $\mathrm{M}_{2,3}$ edge. 


\section{Chapter 5}

\section{Conclusion}

Multilayer optics for extreme ultraviolet radiation have led to advancements in many areas in science and technology. This thesis presented tools for the design and fabrication of EUV multilayers for use with femtosecond and attosecond pulses. Multilayers for bandwidth control, optimization of pulse compression and shaping, tri-material multilayers, and transmission multilayers for polarization control were presented. Developments and optimizations for aperiodic multilayers demonstrated great promise for phase control and pulse shortening of extreme ultraviolet light on a femtosecond/attosecond timescale. Tri-material multilayers for EUV photons in the 25-50 eV photon energy range obtained larger spectral bandwidths without sacrificing peak reflectivity compared to bi-material multilayers. They extend the capability of high harmonic sources for dynamic experiments. Transmission multilayers add the ability to adjust the polarization of a femtosecond/attosecond EUV pulse and allow 
for probing of complex material interactions such as spin dynamics.

Metrologies using total electron yield, described in this thesis, enable the full phase characterization of a multilayer optic. This knowledge is critical to ensure that femtosecond and attosecond pulsed do not broaden in time. This knowledge will also be useful for designing multilayer optics that will produce unique pulse structures.

Participation in the first published measurements of a sub-100 attosecond pulses[3] was described. This included developments in EUV optical design and pump/probe measurements using a FROG-CRAB setup. These 80 attosecond probe pulses will continue to contribute to dynamical studies of gases and solids. These measurements were done in collaboration with the Max-Planck institute for Quantum Optics and Ludwig-Maximilians University in Garching, Germany, led by Professors Krausz and Kleineberg.

Preliminary results in the pioneering work on spin dynamics using EUV pump/probe techniques have been presented. This included optics development for polarization controlled probing of M-edge transitions in Fe and Ni. This ongoing work will enable first of their kind dynamical studies of complex spin systems. Preliminary experiments have demonstrated the feasibility of producing circularly polarized femtosecond EUV pulses from a high harmonic source and demonstrated EUV circular dichroism with a femtosecond source. The first femtosecond EUV spin dynamics measurements are ongoing, with plans for future work.

With new source development and the emerging availability of free electron lasers, 
critical to scientific contributions will be developments in EUV/SXR optics and metrologies for these new pulsed sources. The multilayer designs described in this thesis can be extended to the higher photon energies that become available with femtosecond duration and can be used with those sources to enable these new scientific studies.

This work was supported by the Director, Office of Science, of the U.S. Department of Energy under Contract No. DE-AC02-05CH11231. 


\section{Bibliography}

[1] D. Attwood. Soft X-Rays and Extreme Ultraviolet Radiation: Principles and Applications. Cambridge University Press, 1999.

[2] M. Cardona and L. Ley. Photoemission in solids I : general principles. Topics in Applied Physics, v. 26. Springer-Verlag, 1978.

[3] E. Goulielmakis, M. Schultze, M. Hofstetter, V. S. Yakovlev, J. Gagnon, M. Uiberacker, A. L. Aquila, E. M. Gullikson, D. T. Attwood, R. Kienberger, F. Krausz, and U. Kleineberg. Single-cycle nonlinear optics. Science, 320(5883):1614, June 2008.

[4] W. Chao, B. D. Harteneck, J. A. Liddle, E. H. Anderson, and D. T. Attwood. Soft x-ray microscopy at a spatial resolution better than $15 \mathrm{~nm}$. Nature, 435:1210, June 2005.

[5] A. Sakdinawat and Y. Liu. Soft-x-ray microscopy using spiral zone plates soft-xray microscopy using spiral zone plates soft-x-ray microscopy using spiral zone plates. Optics Letters, 32(18):2635, 2007. 
[6] E. Spiller. Reflective multilayer coatings for the far uv region. Applied Optics, 15:2333, 1976.

[7] J. H. Underwood and Jr. T. W. Barbee. Layered synthetic microstructures as bragg diffractors for x-ray and extreme ultraviolet: theory and predicted performance. Applied Optics, 20(17):3027, September 1981.

[8] J. H. Underwood and Jr. T. W. Barbee. Soft x-ray imaging with a normal incidence mirror. Nature, 294:429, 1981.

[9] C. W. Gwyn, R. Stulen, D. Sweeney, and D. Attwood. Extreme ultraviolet lithography. Journal of Vacuum Science and Technology B, 16(6):3142, November 1998 .

[10] R. Soufli, D. L. Windt, J. C. Robinson, S. L. Baker, E. Spiller, F. J. Dollar, A. L. Aquila, E. M. Gullikson, B. Kjornrattanawanich, J. F. Seely, and L. Golub. Development and testing of EUV multilayer coatings for the atmospheric imaging assembly instrument aboard the solar dynamics observatory. SPIE, 5901:59010M, 2005.

[11] F.K Richtmyer, E.H Kennard, and T. Lauristen. Introduction to Modern Physics. McGraw-Hill Book Company, 1959.

[12] G. Borrmann. Uber extinktions diagramme von quartz. Physikol Z., 42:157, 1941. 
[13] A. V. Vinogradov and B. Ya. Zeldovich. X-ray and far uv multilayer mirrors: principles and possibilities. Applied Optics, 16(1):89-93, 1977.

[14] A.L. Aquila, F. Salmassi, E.M. Gullikson, F. Eriksson, and J. Birch. Measurements of the optical constants of Scandium in the 50-1300ev range. SPIE, 5538(64), 2004.

[15] Yu. A. Uspenskii, J. F. Seely, N. L. Popov, A. V. Vinogradov, Yu. P. Pershin, and V. V. Kondratenko. Efficient method for the determination of extremeultraviolet optical constants in reactive materials: application to scandium and titanium. Journal of the Optical Society of America A, 21(2):298, 2004.

[16] B.L. Henke, E.M. Gullikson, and J.C. Davis. X-ray interactions: photoabsorption, scattering, transmission, and reflection at $\mathrm{e}=50-30000 \mathrm{ev}, \mathrm{Z}=1-92$. Atomic Data and Nuclear Data Tables, 54:181, 1993.

[17] M. Altarelli, D. L. Dexter, H. M. Nussenzveig, and D. Y. Smith. Superconvergence and sum rules for the optical constants. Physical Review B, 6:4502, 1972.

[18] J. B. Kortright and D. L. Windt. Amorphous silicon carbide coatings for extreme ultraviolet optics. Applied Optics, 27(14):2841-2846, 1988.

[19] E. Spiller. Interference in thin films: theory in Soft X-ray Optics. SPIE Optical Engineering Press, 1994. 
[20] R. A. M. Keski-Kuha, J. F. Osantowski, H. Herzig, J. S. Gum, and A. R. Toft. Normal incidence reflectance of ion beam deposited SiC films in the EUV. Applied Optics, 27(14):2815-2816, 1988.

[21] S.V. Gaponov, S.A. Gusev, B.M. Luskin, N.N. Salashchenko, and E.S. Gluskin. Long-wave x-ray radiation mirrors. Optics Communications, 38(1):7-9, 1981.

[22] E. M. Gullikson, S. Mrowka, and B. B. Kaufmann. Recent developments in euv reflectometry at the Advanced Light Source. SPIE, 4343:363, 2001.

[23] A. H. Zewail. Femtochemistry: Atomic-scale dynamics of the chemical bond. Journal of Physical Chemistry A, 104:5660, 2000.

[24] T. Brabec and F. Krausz. Intense few-cycle laser fields: Frontiers of nonlinear optics. Reviews of Modern Physics, 72(2):545, 2000.

[25] A. Rousse, C. Rischel, and J. C. Gauthier. Colloquium: Femtosecond x-ray crystallography. Review of Modern Physics, 73:17, 2001.

[26] M. Drescher, M. Hentschel, R. Kienberger, M. Uiberacker, V. Yakovlev, A. Scrinzi, Th. Westerwalbesloh, U. Kleineberg, U. Heinzmann, and F. Krausz. Time-resolvedatomicinner-shell spectroscopy. Nature, 419:803, 2002.

[27] M. Hentschel, R. Kienberger, Ch. Spielmann, G. A. Reider, N. Milosevic, T. Brabec, P. Corkum, U. Heinzmann, M. Drescher, and F. Krausz. Attosecond metrology. Nature, 414:509, 2001. 
[28] R. Kienberger, M. Hentschel, M. Uiberacker, Ch. Spielmann, M. Kitzler, A. Scrinzi, M. Wieland, Th. Westerwalbesloh, U. Kleineberg, U. Heinzmann, M. Drescher, and F. Krausz. Steering attosecond electron wave packets with light. Science, 297:1144, 2002.

[29] A Scrinzi, M Yu Ivanov, R Kienberger, and D M Villeneuve. Attosecond physics. Journal of Physics B, 39:R1, 2006.

[30] F. Krausz and M. Ivanov. Attosecond physics. Review of Modern Physics, $81: 163,2009$.

[31] P. H. Bucksbaum. The future of attosecond spectroscopy. Science, 317:766, 2007.

[32] E Muybridge. Animal locomotion: an electro-photographic investigation of consecutive phases of animal movements. JB Lippincott Company, 1887.

[33] H. E. Edgerton. Electronic flash, strobe. by McGraw-Hill, 1970.

[34] P. B. Corkum. Plasma perspective on strong field multiphoton ionization. Physical Review Letters, 71(13):1994, 1993.

[35] R. A. Bartels, A.Paul, H. Green, H. C. Kapteyn, M. M. Murnane, S. Backus, I. P. Christov, Y. Liu, D. Attwood, and C. Jacobsen. Generation of spatially coherent light at extreme ultraviolet wavelengths. Science, 297:376, 2002. 
[36] C. Winterfeldt, C. Spielmann, and G. Gerber. Colloquium: Optimal control of high-harmonic generation. Review of Modern Physics, 80:117, 2008.

[37] Y Mairesse, N Dudovich, J Levesque, M Yu Ivanov, P B Corkum, and D M Villeneuve. Electron wavepacket control with elliptically polarized laser light in high harmonic generation from aligned molecules. New Journal of Physics, 10:025015, 2008.

[38] K. Varjú, P. Johnsson, R. López-Martens, T. Remetter, E. Gustafsson, J. Mauritsson, M. B. Gaarde, K. J. Schafer, Ch. Erny, I. Sola, A. Zaïr, E. Constant, E. Cormier, E. Mével, and A. L'Huillier. Experimental studies of attosecond pulse trains. Laser Physics, 15(6):888-898, 2005.

[39] P. Salières, A. L'Huillier, and M. Lewenstein. Coherence control of high-order harmonics. Physical Review Letters, 74:3776, 1995.

[40] C. Hirlimann. Femtosecond Laser Pulses: Principles and Experiments. Springer, second edition, 2005.

[41] A. M. Weiner. Femtosecond pulse shaping using spatial light modulators. Review Of Scientific Instruments, 71(5):1929, 2000.

[42] M. Nisoli, S. De Silvestri, and O. Svelto. Generation of high energy 10 fs pulses by a new pulse compression technique. Applied Physics Letters, 68:2793, 1996. 
[43] I. Walmsley, L. Waxer, and C. Dorrer. The role of dispersion in ultrafast optics. Review Of Scientific Instruments, 72(1):1, 2001.

[44] A. M. Weiner, J. P. Heritage, and E. M. Kirschner. High-resolution femtosecond pulse shaping. Journal of the Optical Society of America B, 5(8):1563, 1988.

[45] E. Gustafsson, T. Ruchon, M. Swoboda, T. Remetter, E. Pourtal, R. LópezMartens, Ph. Balcou, and A. L'Huillier. Broadband attosecond pulseshaping. Optics Letters, 32(11):1353, June 2007.

[46] K. T. Kim, C. M. Kim, M. G. Baik, G. Umesh, and C. H. Nam. Single sub-50attosecond pulse generation from chirp-compensated harmonic radiation using material dispersion. Physical Review A, 69:051805(R), 2004.

[47] R. L. Fork, 0. E. Martinez, and J. P. Gordon. Negative dispersion using pairs of prisms. Optics Letters, 9(5):150, May 1984.

[48] 0. E. Martinez, J. P. Gordon, and R. L. Fork. Negative group-velocity dispersion using refraction. Journal of the Optical Society of America A, 1(10):1003, October 1984.

[49] N. Matuschek, F.X. Kartner, and U. Keller. Theory of double-chirped mirrors. IEEE Journal of Selected Topics in Quantum Electronics, 4(2):197, 1998.

[50] R. Szipocs, K. Ferencz, C. Spielmann, and F. Krausz. Chirped multilayer coat- 
ings for broadband dispersion control in femtosecond lasers. Optics Letters, 19(3):201, 1994.

[51] R. Szipőcs and A. Kőházi-Kis. Theory and design of chirped dielectric laser mirrors. Applied Physics B, 65(2):1432, 1997.

[52] A. S. Morlens, P. Balcou, P. Zeitoun, C. Valentin, V. Laude, and S. Kazamias. Compression of attosecond harmonic pulses by extreme-ultraviolet chirped mirrors. Optics Letters, 30(12):1554, 2005.

[53] A. Aquila, F. Salmassi, and E. Gullikson. Metrologies for the phase characterization of attosecond extreme ultraviolet optics. Optics Letters, 33:455, 2008.

[54] Z. Wang and A. G. Michette. Broadband multilayer mirrors for optimum use of soft x-ray source output. Journal of Optics A, 2:452, 2000.

[55] S. Yulin, T. Kuhlmann, T. Feigl, and N. Kaiser. Spectral reflectance tuning of euv mirrors for metrology applications. In R.L. Engelstad, editor, Emerging Lithographic Technologies VII, volume 5037, pages 286-293. SPIE, 2003.

[56] S. Bajt, D. Stearns, and P. Kearney. Investigation of the amorphous-tocrystalline transition in Mo/Si multilayers. Journal of Applied Physics, 90:1017, 2001.

[57] R.S. Rosen, D. G. Stearns, M. A. Viliardos, M. E. Kassner, S. P. Vernon, and 
Y. Cheng. Silicide layer growth rates in Mo/Si multilayers. Applied Optics, 32:6975, 1993.

[58] P. D. Binda and F. E. Zocchi. Genetic algorithm optimization of x-ray multilayer coatings. In M. Sanchez del Rio, editor, Advances in Computational Methods for X-Ray and Neutron Optics, volume 5536, pages 97-108. SPIE, 2004.

[59] B. Liao and R. Luus. Comparison of the Luus-Jaakola optimization procedure and the genetic algorithm. Engineering Optimization, 37:381, 2005.

[60] W. H. Press, S. A. Teukolsky, W. T. Vetterling, and B. P. Flanery. Numerical Recipes- the art of scientific computing. Cambridge University Press, 3 edition, 2007.

[61] I. V. Kozhevnikov, I. N. Bukreeva, and E. Ziegler. Design of x-ray supermirrors. Nuclear Insterments and Methods in Physics Research A, 460:424, 2001.

[62] N. Dudovich, O. Smirnova, J. Levesque, Y. Mairesse, M. Yu. Ivanov, D. M. Villeneuve, and P. B. Corkum. Measuring and controlling the birth of attosecond XUV pulses. Nature Physics, 2:781, 2006.

[63] H. Takenaka, S. Ichimaru, T. Ohchi, and E.M. Gullikson. Soft-x-ray reflectivity and heat resistance of $\mathrm{SiC} / \mathrm{Mg}$ multilayer. Journal of Electron Spectroscopy and Related Phenomena, 144:1047, 2005. 
[64] A. L. Aquila, F. Salmassi, F. Dollar, Y. Liu, and E. Gullikson. Developments in realistic design for aperiodic Mo/Si multilayer mirrors. Optics Express, 14:10073, 2006.

[65] J. I. Larruquert. Reflectance enhancement with subquarterwave multilayers of highly absorbing materials. Journal of the Optical Society of America A, $18: 1406,2001$.

[66] J. I. Larruquert. General theory of sub-quarterwave multilayers with highly absorbing materials. Journal of the Optical Society of America A, 18:2617, 2001.

[67] J. Gautier, F. Delmotte, M. Roulliay, F. Bridou, M. F. Ravet, and A. Jérome. Study of normal incidence of three-component multilayer mirrors in the range 20-40 nm. Applied Optics, 44:384, 2005.

[68] P. Boher, L. Hennet, and Ph. Hotidy. Three materials soft x-ray mirrors: theory and application. SPIE, 1345:198, 1990.

[69] Yu. A. Uspenskii, V. E. Levashov, A. V. Vinogradov, A. I. Fedorenko, V. V. Kondratenko, Yu. P. Pershin, E. N. Zubarev, and V. Yu. Fedotov. Highreflectivity multilayer mirrors for a vacuum-ultraviolet interval of $35-50 \mathrm{~nm}$. Optics Letters, 23:771, 1998.

[70] J.B. Kortright and J. H. Underwood. Multilayer optical elements for generation 
and analysis of circularly polarized x-rays. Nuclear Insterments and Methods in Physics Research A, 291:272, 1990.

[71] F. Schäfers, H. C. Mertins, A. Gaupp, W. Gudat, M. Mertin, I. Packe, F. Schmolla, S. Di Fonzo, G. Soullié, W. Jark, R. Walker, X. L. Cann, R. Nyholm, and M. Eriksson. Soft-x-ray polarimeter with multilayer optics: Complete analysis of the polarization state of light. Applied Optics, 38(19):4074, 1999.

[72] J. D. Jackson. Clasical Electrodynamics. John Wiley \& Sons, Inc., third editions edition, 1999.

[73] E. Goulielmakis, V. S. Yakovlev, A. L. Cavalieri, M. Uiberacker, V. Pervak, A. Apolonski, R. Kienberger, U. Kleineberg, and F. Krausz. Attosecond control and measurement: Lightwave electronics. Science, 317:769, 2007.

[74] A. L. Cavalieri, N. Müller, Th. Uphues, V. S. Yakovlev, A. Baltuka, B. Horvath, B. Schmidt, L. Blümel, R. Holzwarth, S. Hendel, M. Drescher, U. Kleineberg, P. M. Echenique, R. Kienberger, F. Krausz, and U. Heinzmann. Attosecond spectroscopy in condensed matter. Nature, 449:1029, 2007.

[75] A Wonisch, U Neuhäusler, N. M. Kabachnik, T Uphues, M. Uiberacker, V. Yakovlev, F. Krausz, M. Drescher, U. Kleineberg, and U. Heinzmann. Design, fabrication, and analysis of chirped multilayer mirrors for reflection of extreme-ultraviolet attosecond pulses. Applied Optics, 45(17):4147, 2006. 
[76] S. V. Pepper. Optical analysis of photoemission. Journal of the Optical Society of America, 60(6):805, 1970.

[77] T. Ejima. Formulae of total electron yield for multilayers: Extension of Pepper's method. Japanese Journal of Applied Physics, 42:6459, 2003.

[78] R. Soufli and E. M. Gullikson. Reflectance measurements on clean surfaces for the determination of optical constants of silicon in the extreme ultraviolet-softx-ray region. Applied Optics, 36(22):5499, 1997.

[79] R. Soufli and E. M. Gullikson. Absolute photoabsorption measurements of molybdenum in the range 60-930 ev for optical constant determination. Applied Optics, 37(10):1713, 1998.

[80] C. Tarrio, R. N. Watts, T. B. Lucatorto, J. M. Slaughter, and C. M. Falco. Optical constants of in situ-deposited films of important extreme-ultraviolet multilayer mirror materials. Applied Optics, 37(19):4100, 1998.

[81] M. P. Seah and W. A. Dench. Quantitative electron spectroscopy of surfaces: A standard data base for electron inelastic mean free paths in solids. Surface and Interface Analysis, 1(1):2, 1979.

[82] J. C. Ashley and V. E. Anderson. Interaction of low-energy electrons with silicon dioxide. Journal of Electron Spectroscopy and Related Phenomena, 24(2):127148, 1981. 
[83] J. C. Fuggle and N. Martensson. Core-level binding energies in metals. Journal of Electron Spectroscopy and Related Phenomena, 21:275, 1980.

[84] J. M. Diels, J. J. Fontaine, I. C. McMichael, and F. Simoni. Control and measurement of ultrashort pulse shapes (in amplitude and phase) with femtosecond accuracy. Applied Optics, 24(9):1270-1282, 1985.

[85] D. J. Kane and R. Trebino. Single-shot measurement of the intensity and phase of an arbitrary ultrashort pulse by using frequency-resolved optical gating. Optics Letters, 18(10):823-825, 1993.

[86] R. Trebino, K. W. DeLong, D. N. Fittinghoff, J. N. Sweetser, M. A. Krumbügel, and B. A. Richman. Measuring ultrashort laser pulses in the time-frequency domain using frequency-resolved optical gating. Review Of Scientific Instruments, 68:3277, 1997.

[87] C. Iaconis and I. A. Walmsle. Spectral phase interferometry for direct electricfield reconstruction of ultrashort optical pulses,. Optics Letters, 23:792-794, 1998.

[88] F. Quéré, J. Itatani, G. L. Yudin, and P. B. Corkum. Attosecond spectral shearing interferometry. Physical Review Letters, 90:073902, 2003.

[89] D. J. Bradley, B. Liddy, and W. E. Sleat. Direct linear measurement of ultra- 
short light pulses with a picosecond streak camera. Optics Communications, 2(8):391-395. 391, 1971.

[90] J. Larsson, Z. Chang, E. Judd, P. J. Schuck, R. W. Falcone, P. A. Heimann, H. A. Padmore, H. C. Kapteyn, P. H. Bucksbaum, M. M. Murnane, R. W. Lee, A. Machacek, J. S. Wark, X. Liu, and B. Shan. Ultrafast x-ray diffraction using a streak-camera detector in averaging mode. Optics Letters, 22(13):1012, 1997.

[91] T. Sekikawa, A. Kosuge, T. Kanai, and S. Watanabe. Nonlinear optics in the extreme ultraviolet. Nature, 430:605-608, 2004.

[92] K. Moribayashi, T. Kagawa, and D. E. Kim. Application of x-ray nonlinear processes to the measurement of 10 fs to sub-ps of x-ray pulses. Journal of Physics B, 38:2187-2194, 2005.

[93] J. Itatani, F. Quéré, G. L. Yudin, M. Yu. Ivanov, F. Krausz, and P. B. Corkum. Attosecond streak camera. Physical Review Letters, 88(17):173903, 2002.

[94] Y. Mairesse and F. Quéré. Frequency-resolved optical gating for complete reconstruction of attosecond bursts. Physical Review A, 71:011401, 2005.

[95] H.G. Muller. Reconstruction of attosecond harmonic beating by interference of two-photon transitions. Applied Physics B, 74 [Suppl.]:S17-S71, 2002.

[96] Y. Mairesse, A. de Bohan, L. J. Frasinski, H. Merdji, L. C. Dinu, P. Monchicourt, P. Breger, M. Kovacev, R. Taïeb, B. Carré, H. G. Muller, 
P. Agostini, and P. Salières. Attosecond synchronization of high-harmonic soft x-rays. Science, 302:1540, 2003.

[97] P. M. Paul, E. S. Toma, P. Breger, G. Mullot, F. Augé, Ph. Balcou, H. G. Muller, and P. Agostini. Observation of a train of attosecond pulses from high harmonic generation. Science, 292:1689, 2001.

[98] E. Beaurepaire, J.-C. Merle, A. Daunois, and J.-Y. Bigot. Ultrafast spin dynamics in ferromagnetic Nickel. Physical Review Letters, 76(22):4250-4253, 1996.

[99] J. Kerr. On rotation of the plane of polarization by reflection from the pole of a magnet. Philosophical Magazine, 3(5):321, 1877.

[100] B. Koopmans, M. van Kampen, J. T. Kohlhepp, and W. J. M. de Jonge. Ultrafast magneto-optics in nickel: Magnetism or optics? Physical Review Letters, $85(4): 844,2000$.

[101] C. Stamm, T. Kachel, N. Pontius, R. Mitzner, T. Quast, K. Holldack, S. Khan, C. Lupulescu, E. F. Aziz, M. Wietstruk, H. A. Dürr, and W. Eberhardt. Femtosecond modification of electron localization and transfer of angular momentum in nickel. Nature Materials, 6:740-743, 2007.

[102] J. Stöhr and H. C. Siegmann. Magnetism from Fundamentals to Nanoscale Dynamics. Springer, 2006. 
[103] A. V. Kimel, R. V. Pisarev, J. Hohlfeld, and Th. Rasing. Ultrafast quenching of the antiferromagnetic order in $\mathrm{FeBO}_{3}$ : Direct optical probing of the phononmagnon coupling. Physical Review Letters, 89(28):287401, 2002.

[104] C. Kittel. Interaction of spin waves and ultrasonic waves in ferromagnetic crystals. Physical Review, 110(4):836-841, 1958.

[105] J. Hohlfeld, E. Matthias, R. Knorren, and K. H. Bennemann. Nonequilibrium magnetization dynamics of Nickel. Physical Review Letters, 78(25):4861-4864, 1997.

[106] A. Scholl, L. Baumgarten, R. Jacquemin, and W. Eberhardt. Ultrafast spin dynamics of ferromagnetic thin films observed by fs spin-resolved two-photon photoemission. Physical Review Letters, 79(25):5146-5149, 1997.

[107] J. Walowski, G. Müller, M. Djordjevic, M. Münzenberg, M. Klaüi, C. A. F. Vaz, and J. A. C. Bland. Energy equilibration processes of electrons, magnons, and phonons at the femtosecond time scale. Physical Review Letters, 101:237401, 2008.

[108] I. Tudosa, C. Stamm, A. B. Kashuba, F. King, H. C. Siegmann, J. Stöhr, G. Ju, B. Lu, and D. Weller. The ultimate speed of magnetic switching in granular recording media. Nature, 428:831-833, 2004.

[109] A. V. Kimel, A. Kirilyuk, F. Hansteen, R. V. Pisarev, and T. Rasing. Nonther- 
mal optical control of magnetism and ultrafast laser-induced spin dynamics in solids. Journal of Physics: Condensed Matter, 19:043201, 2007.

[110] C. D. Stanciu, F. Hansteen, A. V. Kimel, A. Kirilyuk, A. Tsukamoto, A. Itoh, and Th. Rasing. All-optical magnetic recording with circularly polarized light. Physical Review Letters, 99:047601, 2007.

[111] B. Koopmans, J. J. M. Ruigrok, F. Dalla Longa, and W. J. M. de Jonge. Unifying ultrafast magnetization dynamics. Physical Review Letters, 95:267207, 2005

[112] B. Koopmans, H.H.J.E. Kicken, M. van Kampen, and W.J.M. de Jonge. Microscopic model for femtosecond magnetization dynamics. Journal of Magnetism and Magnetic Materials, 286:271-275, 2005.

[113] T. L. Gilbert. A phenomenological theory of damping in ferromagnetic materials. IEEE TRANSACTIONS ON MAGNETICS, 40(6):3443, 2004.

[114] H.-S. Rhie, H. A. Dürr, and W. Eberhardt. Femtosecond electron and spin dynamics in ni/w(110) films. Physical Review Letters, 90(24):247201, 2003.

[115] R.W. Schoenlein, S. Chattopadhyay, H.H.W. Chong, T.E. Glover, P.A. Heimann, W.P. Leemans, C.V. Shank, A. Zholents, and M. Zolotorev. Generation of femtosecond x-ray pulses via laser-electron beam interaction. Applied Physics B, 71:1-10, 2000 . 
[116] M. J. Freiser. A survey of magnetooptic effects. IEEE TRANSACTIONS ON MAGNETICS, 4(2):152, 1968.

[117] A. Hubert and R. Schäfer. Magnetic domains. Springer, 1998.

[118] P. Fischer, T. Eimuller, G. Schutz, G. Denbeaux, A. Pearson, L. Johnson, D. Attwood, S. Tsunashima, M. Kumazawa, N. Takagi, M. Kohler, and G. Bayreuther. Element-specific imaging of magnetic domains at $25 \mathrm{~nm}$ spatial resolution using soft x-ray microscopy. Review Of Scientific Instruments, $72(5): 2322-2324,2001$.

[119] G. Schütz, W. Wagner, W. Wilhelm, P. Kienle, R. Zeller, R. Frahm, and G. Materlik. Absorption of circularly polarized x rays in iron. Physical Review Letters, 54(7):737-740, 1987.

[120] C. T. Chen, Y. U. Idzerda, H.-J. Lin, N. V. Smith, G. Meigs, E. Chaban, G. H. Ho, E. Pellegrin, and F. Sette. Experimental confirmation of the x-ray magnetic circular dichroism sum rules for Iron and Cobalt. Physical Review Letters, 75(1):152-155, 1995.

[121] H. Höchst, D. Rioux, D. Zhao, and D. L. Huber. Directional magnetization effects in magnetic circular dichroism spectra of Fe. Physical Review B, 65(6):064439, 2002.

[122] S Valencia, A Gaupp, W Gudat, H-Ch Mertins, P M Oppeneer, D Abramsohn, 
and C M Schneider. Faraday rotation spectra at shallow core levels: 3p edges of Fe, Co, and Ni. New Journal of Physics, 8:254, 2006.

[123] J. B. Kortright and M. Rice. Soft x-ray magneto-optic kerr rotation and element-specific hysteresis measurement. Review Of Scientific Instruments, 67:3353, 1996.

[124] F. U. Hillebrecht, T. Kinoshita, D. Spanke, J. Dresselhaus, Ch. Roth, H. B. Rose, and E. Kisker. New magnetic linear dichroism in total photoelectron yield for magnetic domain imaging. Physical Review Letters, 75(11):2224-2227, 1995.

[125] J. Stöhr. Exploring the microscopic origin of magnetic anisotropies with x-ray magnetic circular dichroism (XMCD) spectroscopy. Journal of Magnetism and Magnetic Materials, 200:470-497, 1999.

[126] P. Carra, B. T. Thole, M. Altarelli, and X. Wang. X-ray circular dichroism and local magnetic fields. Physical Review Letters, 70:694, 1993.

[127] B. L. Mesler, P. Fischer, W. Chao, E. H. Anderson, and D. H. Kim. Soft x-ray imaging of spin dynamics at high spatial and temporal resolution. Journal of Vacuum Science and Technology B, 25(6):2598-2602, 2007.

[128] E. A. Gibson, A. Paul, N. Wagner, R. Tobey, D. Gaudiosi, S. Backus, I. P. Christov, A. Aquila, E. M. Gullikson, D. T. Attwood, M. M. Murnane, and 
H. C. Kapteyn. Coherent soft x-ray generation in the water window with quasiphase matching. Science, 302:95-98, 2003.

[129] S. Eisebitt, J. Lüning, W. F. Schlotter, M. Lörgen, O. Hellwig, W. Eberhardt, and J. Stöhr. Lensless imaging of magnetic nanostructures by x-ray spectroholography. Nature, 432:885-888, 2004.

[130] W. Ackermann and et. al. Operation of a free-electron laser from the extreme ultraviolet to the water window. Nature Photonics, 1:336 - 342, 2007.

[131] H. N. Chapman, A. Barty, M. J. Bogan, S. Boutet, M. Frank, S. P. HauRiege, S. Marchesini, B. W. Woods, S. Bajt, W. Henry Benner, R. A. London, E. Plönjes, M. Kuhlmann, R. Treusch, S. Düsterer, T. Tschentscher, J. R. Schneider, E. Spiller, T. Möller, C. Bostedt, M. Hoener, D. A. Shapiro, K. O. Hodgson, D. van der Spoel, F. Burmeister, M. Bergh, C. Caleman, G. Huldt, M. M. Seibert, F. R. N. C. Maia, Ri. W. Lee, A. Szöke, N. Timneanu, and J. Hajdu. Femtosecond diffractive imaging with a soft-x-ray free-electron laser. Nature Physics, 2:839-843, 2006.

[132] J. Arthur. Status of the LCLS x-ray FEL program. Review Of Scientific Instruments, 73:1393, 2002.

[133] D. Cocco, M. Zangrando, A. Abrami, A. Bianco, I. Cudin, C. Fava, D. Giuressi, R. Godnig, F. Parmigiani, B. Ressel, L. Rumiz, R. Sergo, A. Simoncig, 
C. Svetina, and G. Zgrablic. The fermi@elettra fel photon transport system. SPIE, 7361:7361-05, 2009.

[134] J. A. Bearden and A. F. Burr. Reevaluation of x-ray atomic energy levels. Review of Modern Physics, 39:125, 1967.

[135] S B Qadri, C Kim, M Twigg, and D Moon. Ion-beam deposition of Zr-Al multilayers and their structural properties. Surface and Coatings Technology, 54(1):335-337, 1992.

[136] G. R. Fowle. Introduction to Modern Optics. Courier Dover Publications, second edition, 1989.

[137] X. Zhang, A. L. Lytle, T. Popmintchev, X. Zhou, H. C. Kapteyn, M. M. Murnane, and O. Cohen. Quasi-phase-matching and quantum-path control of highharmonic generation using counterpropagating light. Nature Physics, 3:270-275, 2007. 


\section{Appendix A}

\section{Analytic Modeling of Multilayers}

In this appendix an analytical approximation formula for the peak reflectivity of a multilayer is derived. Starting with the kinematical approximation[19] for multilayers:

$$
\begin{gathered}
r(\omega)=\sum_{j=0}^{2 N-1} r_{j, j+1}(\omega) e^{i 2 \varphi_{j}(\omega)} \\
\varphi_{j}(\omega)=\varphi_{j-1}(\omega)+\frac{2 \pi}{\lambda} n_{j}(\omega) d_{j} \sin \theta
\end{gathered}
$$

where $N$ is the number of bilayers, $r_{j, j+1}(\omega)$ is the complex Fresnel reflection coefficient, $n_{j}=1-\delta_{j}+i \beta_{j}$ is the index of refraction, and $\varphi$ is the phase factor described by equation A.2 (also $\varphi_{0}=0$ ). Equation A.1 is an approximation that only single reflections are taken into account. It is a good approximation as long as $\left|r_{j, j+1}^{2}\right| \ll 1$. If the multilayer is limited to normal incidence $\theta=\frac{\pi}{2}$ and limit the case to two alternating materials two simplifications can be made: $\sin \theta=1$ and $r_{j, j+1}=e^{i \pi} r_{j+1, j+2}$. 
For the peak reflectivity the multilayer must satisfy the Bragg equation:

$$
\begin{gathered}
m \lambda_{0}=2 d \sin \theta \\
d=\frac{\lambda_{0}}{2}
\end{gathered}
$$

where $d$ is the optical thickness of one bilayer. One more simplification is made that the optical depth of each layer $d_{j}=\frac{\lambda_{0}}{4}$ (e.g. quarter-wave stack). Equation A.1 now becomes:

$$
r(\omega)=|r(\omega)| \sum_{j=0}^{2 N-1} e^{i j \pi} e^{i 2 \sum_{k=0}^{j} \frac{2 \pi}{\lambda_{0}} \frac{\lambda_{0}}{4} n_{j}(\omega)}
$$

A simplification can be made if it is assumed that the index of reflectivity is constant, and $\delta=0, \beta_{j}=\beta_{j+1}=\bar{\beta}$ then:

$$
r=|r| \sum_{j=0}^{2 N-1} e^{i 2 \pi j} e^{-j \pi \bar{\beta}}
$$

One can recognize that $e^{i 2 \pi j}=1$ for all $j$ and the sum is a geometric series of finite terms: $\sum_{j=0}^{n-1} r^{j}=\frac{1-r^{n}}{1-r}$. Then:

$$
\begin{gathered}
r=|r| \frac{1-e^{-2 N \pi \bar{\beta}}}{1-e^{-\pi \bar{\beta}}} \\
R(N)=|r|^{2} \frac{1-2 e^{-2 N \pi \bar{\beta}}+e^{-4 N \pi \bar{\beta}}}{1-2 e^{-\pi \bar{\beta}}+e^{-2 \pi \bar{\beta}}}
\end{gathered}
$$

The formula for $R(N)$ can be written with two fitting parameters: $|r|^{2}$ being the reflectivity per interface and $\pi \bar{\beta}$ as the average absorption. 


\section{Appendix B}

\section{Polarization of Multilayer}

\section{Quarter-Wave Plate}

The Jones matrix method of polarization[136] will be used to determine the proper azimuthal angle to use the multilayer quarter-wave plate. The quarter wave plate can be represented as a relative phase retarder:

$$
\mathbf{P}=\left[\begin{array}{cc}
T_{s} e^{i \phi_{s}} & 0 \\
0 & T_{p} e^{i \phi_{p}}
\end{array}\right]
$$

where $T_{s}$ is the transmission of s-polarization, $\phi_{s}$ is transmitted phase s-polarization, $T_{p}$ is the transmission of p-polarization, and $\phi_{p}$ is transmitted phase p-polarizion. The electric field for s-polarization can be represented as:

$$
\vec{E}_{s}=|E|\left[\begin{array}{l}
1 \\
0
\end{array}\right]
$$


The matrix $\mathbf{P}$ can be simplified, as we are only concerned with the relative phase difference between s-polarization and p-polarization:

$$
\mathbf{P}=\left|T_{s}\right|\left[\begin{array}{cc}
1 & 0 \\
0 & \frac{T_{p}}{T_{s}} e^{i \Delta \phi}
\end{array}\right]
$$

where $\Delta \phi$ is the relative phase difference. The desired phase difference between spolarization and p-polarization is $\Delta \phi=\pi / 2$ or $-\pi / 2$. To determine the azimuthal angle, $\theta$, the multilayer quarter-wave plate matrix, $\mathbf{P}$, is rotated by a rotation matrix $\mathbf{R}(\theta)$ (where $\theta$ is measured from the $T_{s}$ transmission axis):

$$
\mathbf{P}(\theta)=\left|T_{s}\right|\left[\begin{array}{cc}
\cos \theta & -\sin \theta \\
\sin \theta & \cos \theta
\end{array}\right]\left[\begin{array}{cc}
1 & 0 \\
0 & e^{-i \pi / 2} \frac{T_{p}}{T_{s}}
\end{array}\right]\left[\begin{array}{cc}
\cos \theta & \sin \theta \\
-\sin \theta & \cos \theta
\end{array}\right]
$$

$\theta$ can be solved for the production of left circularly polarized light produced form s-polarized light:

giving equations:

$$
\begin{array}{r}
|a|\left[\begin{array}{l}
1 \\
i
\end{array}\right]=\left|T_{s}\right|\left[\begin{array}{ll}
\cos \theta & -\sin \theta \\
\sin \theta & \cos \theta
\end{array}\right]\left[\begin{array}{cc}
1 & 0 \\
0 & -i \frac{T_{p}}{T_{s}}
\end{array}\right]\left[\begin{array}{cc}
\cos \theta & \sin \theta \\
-\sin \theta & \cos \theta
\end{array}\right]\left[\begin{array}{l}
1 \\
0
\end{array}\right] \\
|a|\left[\begin{array}{l}
1 \\
i
\end{array}\right]=\left|T_{s}\right|\left[\begin{array}{c}
\cos ^{2} \theta-i \sin ^{2} \theta \frac{T_{p}}{T_{s}} \\
\sin \theta \cos \theta+i \sin \theta \cos \theta \frac{T_{p}}{T_{s}}
\end{array}\right]
\end{array}
$$

$$
\begin{gathered}
|a|=\left|T_{s}\right|\left(\cos ^{2} \theta-i \sin ^{2} \theta \frac{T_{p}}{T_{s}}\right) \\
i|a|=\left|T_{s}\right|\left(\sin \theta \cos \theta+i \sin \theta \cos \theta \frac{T_{p}}{T_{s}}\right)
\end{gathered}
$$

by multiplying equation (B.7) by $i$ and substituting into equation (B.8)

$$
\left|T_{s}\right|\left(+\sin ^{2} \theta \frac{T_{p}}{T_{s}}+i \cos ^{2} \theta\right)=\left|T_{s}\right|\left(\sin \theta \cos \theta+i \sin \theta \cos \theta \frac{T_{p}}{T_{s}}\right)
$$


Solving for either the imaginary or real part yields the same result:

$$
\frac{T_{p}}{T_{s}}=\frac{\cos \theta}{\sin \theta} \Rightarrow \frac{T_{s}}{T_{p}}=\tan \theta
$$

Showing that the desired angle is $\arctan \frac{T_{s}}{T_{p}}=\theta_{a z}$. 


\section{Appendix C}

\section{Semi-Analytical Solution for}

\section{Chirped Multilayers}

This section is an attempt to justify ${ }^{1}$ why quadratically depth-graded multilayers are used to compensate for GDD or chirp. The mirror reflectivity function $r(\omega)$ relates to the impulse response of the multilayer optic through the Fourier transform:

$$
r(\omega)=\int_{0}^{\infty} r(t) e^{-i \omega t} \mathrm{~d} t=\int_{0}^{\infty}|r(t)| e^{i \phi(t)} e^{-i \omega t} \mathrm{~d} t
$$

where $|r(t)|$ is the magnitude of the impulse response and $\phi(t)$ is the phase response. For a chirped mirror (designed to cancel a chirp of an incoming pulse) the lowest order term in $\phi$ (related to the GDD) goes as $a t^{2}$ where $a$ is a constant. The impulse

\footnotetext{
${ }^{1}$ This is not an attempt to be a formal proof.
} 
response can be approximated as:

$$
r(\omega) \approx \sum_{t}|r(t)| e^{i \phi(t)} e^{-i \omega t}
$$

Using the kinematical approximation (from appendix A) we obtain:

$$
r(\omega) \approx \sum_{j=0}^{2 N-1} r_{j, j+1}(\omega) e^{2 i \sum_{k=0}^{j} \frac{2 \pi}{\lambda} n_{k}(\omega) d_{k} \sin \theta}
$$

Solving for normal incidence $\sin \theta=1$ and using the approximations that the index of refraction for both materials are constant $r_{j, j+1}=|r| e^{i \pi j}$ and $n=1+i \bar{\beta}$ then equation (C.3) becomes:

$$
r(\omega) \approx \sum_{j=0}^{2 N-1}|r| e^{-2 \frac{2 \pi \bar{\beta}}{\lambda} \sum_{k=0}^{j} d_{k}} e^{i\left(\pi j+2 \frac{2 \pi}{\lambda} \sum_{k=0}^{j} d_{k}\right)}
$$

For the absorptive (real exponential) we can approximate the absorption to that of a quarter-wave stack. A depth function can be defined $\sum_{k=0}^{j} d_{k} \equiv z[j]$ Equation (C.4) then reduces to $^{2}$ :

$$
r(\omega) \approx \sum_{j=0}^{2 N-1}|r| e^{\pi \bar{\beta} j} e^{i\left(\pi j+\frac{2 \omega}{c} z[j]\right)}
$$

The depth function $z[j]$ can be converted into a time function of the phase velocity $z[j]=V_{\text {phase }} \tau[j]$ in the EUV we approximate $V_{\text {phase }}=c$.

$$
r(\omega) \approx \sum_{j=0}^{2 N-1}|r| e^{\pi \bar{\beta} j} e^{i(\pi j+2 \omega \tau[j])}
$$

The impulse response equation (C.2) must equal equation (C.6) as both equations equal $r(\omega)$ :

$$
\sum_{t}|r(t)| e^{i \phi(t)} e^{-i \omega t} \approx \sum_{j=0}^{2 N-1}|r| e^{\pi \bar{\beta} j} e^{i(\pi j+2 \omega \tau[j])}
$$

\footnotetext{
${ }^{2} 2 \pi / \lambda=\omega / c$ is being substituted for later convenience.
} 
The complex phases of equation (C.7) must be equal.

$$
\phi(t)-\omega t \approx \pi j+2 \omega \tau[j] \Rightarrow \phi / 2 \omega+t / 2-\pi j / 2 \omega=\tau[j]=c z[j]
$$

For a chirped pulse:

$$
z[j]=a t^{2} / 2 \omega c+t / 2 c-\pi j / 2 \omega c
$$

This equation implies that a quadratically depth grated multilayer (with layer thickness $d[j]=z[j+1]-z[j])$ will produce a chirped multilayer. 